\title{
Two Magnon Excitations in the Heisenberg Ferromagnet on the Triangular Lattice
}

\author{
by \\ Xiaohua Qu
}

\author{
A thesis \\ presented to the University of Manitoba \\ in partial fulfillment of the \\ requirements for the degree of \\ Master of Science \\ in
}

Physics

Winnipeg, Manitoba

(c) Xiaohua Qu, 1989 
The author has granted an irrevocable nonexclusive licence allowing the National Library of Canada to reproduce, loan, distribute or sell copies of his/her thesis by any means and in any form or format, making this thesis available to interested persons.

The author retains ownership of the copyright in his/her thesis. Neither the thesis nor substantial extracts from it may be printed or otherwise reproduced without his/her permission.
L'auteur a accordé une licence irrévocable et non exclusive permettant à la Bibliothèque nationale du Canada de reproduire, prêter. distribuer ou vendre des copies de sa thèse de quelque manière et sous quelque forme que ce soit pour mettre des exemplaires de cette thèse à la disposition des personnes intéressées.

L'auteur conserve la propriété du droit d'auteur qui protège sa thèse. Ni la thèse ni des extraits substantiels de celle-ci ne doivent être imprimés ou autrement reproduits sans son autorisation. 
TWO MAGNON EXCITATIONS IN THE HEISENBERG

FERROMAGNET ON THE TRIANGULAR LATTICE

BY

XIAOHUA QU

A thesis submitted to the Faculty of Graduate Studies of

the University of Manitoba in partial fulfillment of the requirements of the degree of

MASTER OF SCIENCE

(c) 1989

Permission has been granted to the LIBRARY OF THE UNIVER-

SITY OF MANITOBA to lend or sell copies of this thesis, to the NATIONAL LIBRARY OF CANADA to microfilm this thesis and to lend or sell copies of the film, and UNIVERSITY MICROFILMS to publisin an abstract of this thesis.

The author reserves other publication rights, and neither the thesis nor extensive extracts from it may be printed or otherwise reproduced without the author's written permission. 


\begin{abstract}
The spectrum of two-magnon excitations are examined for a 2-D triangular Heisenberg ferromagnet with various values of $\operatorname{spin}(S), \operatorname{Ising}(\sigma)$ and uniaxial $(D)$ anisotropies and for all total wave vectors $\vec{K}$ in three high symmetry directions. In contrast to earlier studies of hypercubic ferromagnets(NN chain, square and simple cubic), the width of the continuum never goes to zero, rather reminiscent of the NN FCC case, and therefore the $S, \sigma, D$ parameters are found to be very significant to the behaviour of s-, d-, single-ion bound states, as well as the continuum. The internal van Hove singularities also play an important role in the evolution of resonances. Finally the results are compared with a Raman scattering experiment in $\mathrm{FeBr}_{2}$.
\end{abstract}




\section{Acknowledgement}

Foremost, I would like to thank Professor Peter Loly for his help, encouragement and guidance that made this thesis possible, also for his understanding and patience when the writing of thesis was delayed since my involvement in the pro-democratic movement for China. Secondly, I would like to thank my parents for all their efforts throughout my life. I would like to thank Professor R. Wong and Tom Osborn for their help extended to me both before and after my arrival in Canada. I am also grateful to the examining committee for their time they gave to read and examine this thesis. Finally, the contribution of the University of Manitoba through its fellowship is acknowledged. 


\section{Contents}

1 Introduction 1

2 The Two-magnon Problem and the Green Function Method 11

2.1 Description of Two-magnon States . . . . . . . . . 11

2.2 Double-time Green Functions $\ldots \ldots \ldots \ldots \ldots$

2.3 Dyson's Equation of the Two-magnon Propagator . . . . . . . . 18

3 Application to the Triangular Lattice 26

3.1 Two-Magnon Propagators with NN Interaction . . . . . . . . 26

3.2 Critical Points in the Two-magnon Spectra . . . . . . . . . 34

3.3 Lattice Green Functions . . . . . . . . . . . . . . . . . 40

4 Results and Discussion 48

4.1 Two-Magnon Spectrum in the Absence of

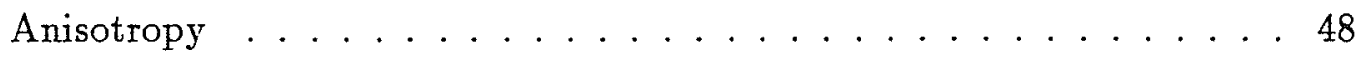

4.2 Two-Magnon spectrum with Various Anisotropies . . . . . . . . 59

4.3 Raman Scattering . . . . . . . . . . . . . . . . . . 74 
A

A.1 Program for Critical lines . . . . . . . . . . . . . . . 80

A.2 Program for Two-Magnon spectrum at $\vec{K}=0 \ldots \ldots$. . . . . . 84

A.3 Program for Two-Magnon Spectrum at gerenal $\vec{K}$ 's . . . . . . . . 93 


\section{List of Figures}

1.1 Isotropic Two-magnon Dispersion relation . . . . . . . . . . 8

3.1 The real space network of the triangular lattice . . . . . . . . 27

3.2 The basic cell of the real space lattice and 1-st the brillouin Zone . . 35

3.3 Critical Points . . . . . . . . . . . . . . . 41

3.4 Lattice Green's Functions Calculated in Terms of Elliptic Integrals with Horiguchi's Method ................. . 45

3.5 Lattice Green's Functions Calculated Numerically using the Triangular Linear Analytic Method . . . . . . . . . . . . . . 46

4.1 Two-magnon spectra at $K=0$ in the absence of anisotropy . . . 50

4.2 Ising energy levels in the absence of anisotropy $(\sigma=0.1, D=0$ and

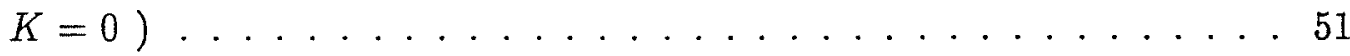

4.3 Dispersion of resonance peaks and bound states for $S=1$ in the absence of anisotropy ..................... 52

4.4 The evolution of two-magnon propagators in the absence of anisotropy 53

4.5 Dispersion of Bound States for $S=1$ and $S=\frac{1}{2} \ldots \ldots$ 
4.6 Two-magnon spectra at $K=0$ for $D=0, \sigma=0.78 \ldots \ldots 2$

4.7 Two-magnon spectra at $K=0$ for $D=2.26, \sigma=0.0 \ldots \ldots 3$

4.8 Two-magnon spectra at $K=0$ for $D=2.26, \sigma=0.78 \ldots \ldots 64$

4.9 Dispersion of resonance peaks and bound states for $S=1$ and $D=0, \sigma=0.78 \ldots \ldots \ldots 6 . \ldots \ldots$

4.10 Dispersion of resonance peaks and bound states for $S=1$ and $D=2.26, \sigma=1.0 \ldots \ldots \ldots 6$

4.11 Dispersion of resonance peaks and bound states for $S=1$ and $D=2.26, \sigma=0.78 \ldots \ldots \ldots 67$

4.12 The evolution of two-magnon propagators for $D=0.0, \sigma=0.78 \quad$. 68

4.13 The evolution of two-magnon propagators for $D=2.26, \sigma=1.0 \quad$. 70

4.14 The evolution of two-magnon propagators for $D=2.26, \sigma=0.78 \ldots 72$ 


\section{Chapter 1}

\section{Introduction}

The wonders of magnetism have been challenging the mind of man ever since the times of the ancient Chinese "Emperor Huang" [1], [2] and Greek civilizations [3]. However, most of important experimental and theoretical discoveries have been made only since the turn of century, especially since the birth of quantum mechanics, so that magnetism becomes one of the most active and complex fields of modern physics that we see today. The models used as well as theoretical approaches vary widely between different magnetic properties of varying magnetic materials. Paramagmetism and diamagnetism may be at least qualitatively understood in terms of the statistical average of properties of individual atoms, ions, or electrons by assuming these discrete sources of magnetic moment do not interact with each other. Then there is no net magnetic moment under zero external field. On the other hand, various phenomena of magnetic ordering below a critical temperature $T_{c}$, classified as: ferromagnetism, antiferromagnetism, ferrimagnetism, etc., are observed in many materials. The ferromagnet has a nonvanishing spontaneous magnetization, usually oriented to certain 
favored direction, even in the absence of an external magnetic field. This parallel orientation, as well as the spontaneous antiparallel orientation in antiferromagnets then must be due to interactions between individual magnets in solids. It was Weiss (1907) who first successfully took into account the effect of interaction by introducing the 'Weiss internal field' which, as expected, leads to a critical temperature - the Curie temperature for the phase transition between ferro- and para-magnetism.

The theory of the origin of these interactions is of course the first step in a study of magnetic ordering. But in fact it is still a fundamental task of physics to derive a general model of magnetic interactions from basic quantum principles. However, to the first approximation, one needs only consider the Coulomb interaction exchange integral, which is direct consequence of Pauli antisymmetry requirement of the total electron wavefunction. This mechanism was first discussed in 1926 by Heisenberg [4] and Dirac [5].

In insulating solids, where magnetic ions are well separated, all of the electrons are supposed to be bound on their ions and the wavefunction overlaps between different ions are small, the magnetic interaction may be described by the basic Heisenberg Hamiltonian,

$$
H=-\sum_{i j} J_{i j} \vec{S}_{i} \cdot \vec{S}_{j}
$$

where we assign a total spin operator $\vec{S}_{i}$ to the atom on the $\mathrm{i}$-th lattice site and $J_{i j}=J\left(\left|\vec{R}_{i}-\vec{R}_{j}\right|\right)$ denotes the exchange integral coupling the atoms on $\mathrm{i}$-th and $\mathrm{j}$-th lattice sites, which is a function of relative distance between two sites.

The Heisenberg Hamiltonian is at least a successful semi-phenomenological model of 
interacting moments and serves as the starting point of many profound investigations of magnetism in solids, and indeed, the starting point of this thesis.

Taking into account certain anisotropies [6], [7] the basic model can be generalized[8]:

$$
H=-\sum_{i j}\left(I_{i j} S_{i}^{z} S_{j}^{z}+J_{i j} S_{i}^{+} S_{j}^{-}\right)-\sum_{i} D\left(S_{i}^{z}\right)^{2}
$$

where $D$ represents uniaxial single-ion anisotropy and $J_{i j}=0$ gives the well-known Ising model. ${ }^{1}$

The categories of ferromagnets and antiferromagnets are roughly determined by the coefficients $J_{i j}$ (and $I_{i j}$ ). They are positive for ferromagnets (the NN interaction denoted by $J_{1}$ is usually dominant). The simplest antiferromagnet arises when the local moments fall into two interpenetrating sublattices of identical structure when J's are positive within each sublattice but negative between them. In this thesis we will only consider ferromagnets.

Obviously a parallel alignment of spins is favoured in ferromagnets. If there are a total of $\mathrm{N}$ atoms in the system, the ground state is given by assigning the minimum value $-S$ (or the maximum $S$ ) to all spins,

$$
|0>=|-S>_{1}\left|-S>_{2} \cdots\right|-S>_{l} \cdots \mid-S>_{N}
$$

The ground state energy is,

$$
E_{0}=-S^{2} \sum_{i j} J_{i j}
$$

And the magnetization is saturated.

\footnotetext{
${ }^{1}$ it can be further generalized to include some other high order anisotropies $[9],[10],[3],[11]$
} 
The ground state may be excited by switching spins on some lattice site by one unit. Let,

$$
|l>=|-S>_{1}\left|-S>_{2} \cdots\right|-S>_{l-1}\left|-S+1>_{l}\right|-S>_{l+1} \cdots \mid-S>_{N}
$$

The 1st excited energy eigenstate of basic Heisenberg Hamiltonian ( eq. 1.1 ) is a wave-like superposition of these states,

$$
\left|\vec{k}>=\frac{1}{N} \sum_{l}\right| l>e^{i \vec{k} \cdot \vec{R}_{l}}
$$

Where $\vec{R}_{l}$ denotes the position vector of $l$-th site and $\vec{k}$ is a wavevector. The corresponding energy eigenvalue is

$$
E(\vec{k})=E_{0}+\Omega(\vec{k})
$$

Where the dispersion function is given by

$$
\begin{aligned}
\Omega(\vec{k}) & =2 S \sum_{n \neq 0} J\left(\vec{R}_{n}\right)\left(1-e^{i \vec{k} \cdot \vec{R}_{n}}\right) \\
& =2 S[J(0)-J(\vec{k})]
\end{aligned}
$$

where we carried out a transformation,

$$
J(\vec{k})=\sum_{l} \exp ^{i \vec{k} \cdot \vec{R}_{l}} J\left(\vec{R}_{l}\right)
$$

with inverse

$$
J_{i j}=\frac{1}{N} \sum_{\vec{q}} J(\vec{q}) \exp \left(-i \vec{q} \cdot\left(\vec{r}_{i}-\vec{r}_{j}\right)\right)
$$

This is the so-called spin wave state. Like lattice waves - the normal modes of lattice vibration, spin deviations in such a state spread over the whole system as a plane 
wave rather than localize on some particular lattice sites. The spins at different sites precess with a phase shift about the direction of the magnetic alignment.

In the language of elementary excitations, we introduce creation and annihilation operators $a_{i}^{+}, a_{i}^{-}$which add or diminish one unit of spin deviation of $i$-th spin when being applied on the system.

$$
\begin{aligned}
S_{i}^{+} & =(2 S)^{\frac{1}{2}} a_{i}^{+}\left(1-\frac{a_{i}^{+} a_{i}^{-}}{2 S}\right)^{\frac{1}{2}} \\
S_{i}^{-} & =(2 S)^{\frac{1}{2}}\left(1-\frac{a_{i}^{+} a_{i}^{-}}{2 S}\right)^{\frac{1}{2}} a_{i}^{-} \\
S_{i}^{z} & =-S+a_{i}^{+} a_{i}^{-}
\end{aligned}
$$

where $a_{i}^{+}, a_{i}^{-}$satisfy the boson type commutation relation.

$$
\left[a_{i}^{+} ; a_{j}^{-}\right]=\delta_{i j}, \quad\left[a_{i}^{+} ; a_{j}^{+}\right]=\left[a_{i}^{+} ; a_{j}^{+}\right]=0
$$

Through the exchange interaction, spin deviations at any particular site in real space are propagated through whole spin system. We therefore have to include creation and annihilation operators of the spin wave quanta. They are Fourier transforms of real space operators,

$$
\begin{aligned}
& a_{\vec{k}}^{+}=\frac{1}{N} \sum_{i} a_{i}^{+} \exp \left(i \vec{k} \cdot \vec{R}_{i}\right) \\
& a_{\vec{k}}^{-}=\frac{1}{N} \sum_{i} a_{i}^{-} \exp \left(-i \vec{k} \cdot \vec{R}_{i}\right)
\end{aligned}
$$

Applying $a_{\vec{k}}^{+}$on the ground state,

$$
\begin{aligned}
a_{\vec{k}}^{+} \mid 0> & =\frac{1}{N} \sum_{l} \exp \left(i \vec{k} \cdot \vec{R}_{l}\right) a_{l}^{+} \mid 0> \\
& =\frac{1}{N} \sum_{l} \mid l>\exp \left(i \vec{k} \cdot \vec{R}_{l}\right)
\end{aligned}
$$


We see that it does create a spin wave state. Therefore a spin wave may be associated with an elementary quanta-a magnon with energy of a basic unit $\hbar \Omega(\vec{k})$, and "momentum" $\hbar \vec{k}$. The operator $\left(a_{\vec{k}}^{+} a_{\vec{k}}^{-}\right)$represents the occupation number of the magnon $(\vec{k}, \Omega(\vec{k}))$. Then the whole spin system may be described as a 'magnon gas system'.

The Heisenberg Hamiltonian can be second quantized in the occupation number representation,

$$
\begin{aligned}
H=- & \sum_{i j} J_{i j} \vec{S}_{i} \cdot \vec{S}_{j} \\
=E_{0} & +\sum_{\vec{k}} \Omega(\vec{k}) a_{\vec{k}}^{+} a_{\vec{k}}^{-} \\
& \quad+\frac{1}{2 N} \sum_{\vec{k} \vec{k}^{\prime} \vec{q}}\left(J(\vec{k})+J(\vec{k}+q)-2 \Omega\left(\vec{k}+\vec{q}-\vec{k}^{\prime}\right)\right) a_{\vec{k}-\vec{q}^{\prime}}^{+} a_{\vec{k}^{\prime}+\vec{q}}^{+} a_{\vec{k}^{\prime}}^{-} a_{\vec{k}}^{-}+\ldots
\end{aligned}
$$

The second term is the sum of contributions from 'independent' magnons. The third term describes the interaction between magnons through a two-magnon scattering process.

Considering only the second term, we will get the so called 'free magnon' model. The energy of the non-interacting two-magnon state is therefore given by,

$$
\begin{aligned}
\Omega\left(\vec{k}_{1}, \vec{k}_{2}\right) & =\Omega\left(\vec{k}_{1}\right)+\Omega\left(\vec{k}_{2}\right) \\
& =2 S\left[2 J(0)-J\left(\vec{k}_{1}\right)-J\left(\vec{k}_{2}\right)\right]
\end{aligned}
$$

or,

$$
\Omega(\vec{K}, \vec{k})=2 S\left[2 I(0)-J\left(\frac{1}{2} \vec{K}+\vec{k}\right)-J\left(\frac{1}{2} \vec{K}-\vec{k}\right)\right]+2(2 S-1) D
$$


where we define the total and relative wavevectors,

$$
\vec{K}=\vec{k}_{1}+\vec{k}_{2}, \quad \vec{k}=\left(\vec{k}_{1}-\vec{k}_{2}\right) / 2
$$

Unfortunately such a non-interacting two-magnon state is not an eigenstate of Heisenberg Hamiltonian because of the scattering between magnons rising from high order terms.

Let us use $\mid i j>$ to represent the state in which one unit deviation occurred at each of $\mathrm{i}$-th and $\mathrm{j}$-th sites, $\mid i i>$ to represent two unit deviations coinciding at the $\mathrm{i}$-th lattice site. In general a two-magnon eigenstate can be expressed as,

$$
\left|E, \vec{K}>=\sum_{i j} e^{i \vec{K} \cdot\left(r_{i}+r_{j}\right) / 2} F\left(\left|r_{i}-r_{j}\right|\right)\right| i j>
$$

Here the $\mid E, \vec{K}>$ represents the two-magnon energy eigenstate, and $F\left(r_{i j}\right)$ gives the weight coefficient of the $\mid i j>$ state. The detailed explanation of this equation and the interating two-magnon problem will be given in chapter 2 .

Taking into consideration the magnon interaction, the coefficient $F(r)$ also changes from the 'free magnon' case, and we will find that the most significant effect is the emergence of the so-called "bound state" branches, usually located below the independent two-magnon continuum. The bound state derives its name from the fact that it is composed of two-magnon states in which spin deviations are located on near neighbours, in other words $F(r)$ converges quickly as $|\vec{r}|$ increases.

A plot of the isotropic two-magnon dispersion relationship for an infinite chain ferromagnet (Fig. 1.1) illustrates the bound states as well as the continuum, which coincides with the the allowed region of non-interacting magnon pairs In NN (nearest 


\section{TWO-MAGNON DISPERSION RELATIONSHIP ISOTROPIC, SPIN $=1 / 2$ CASE}

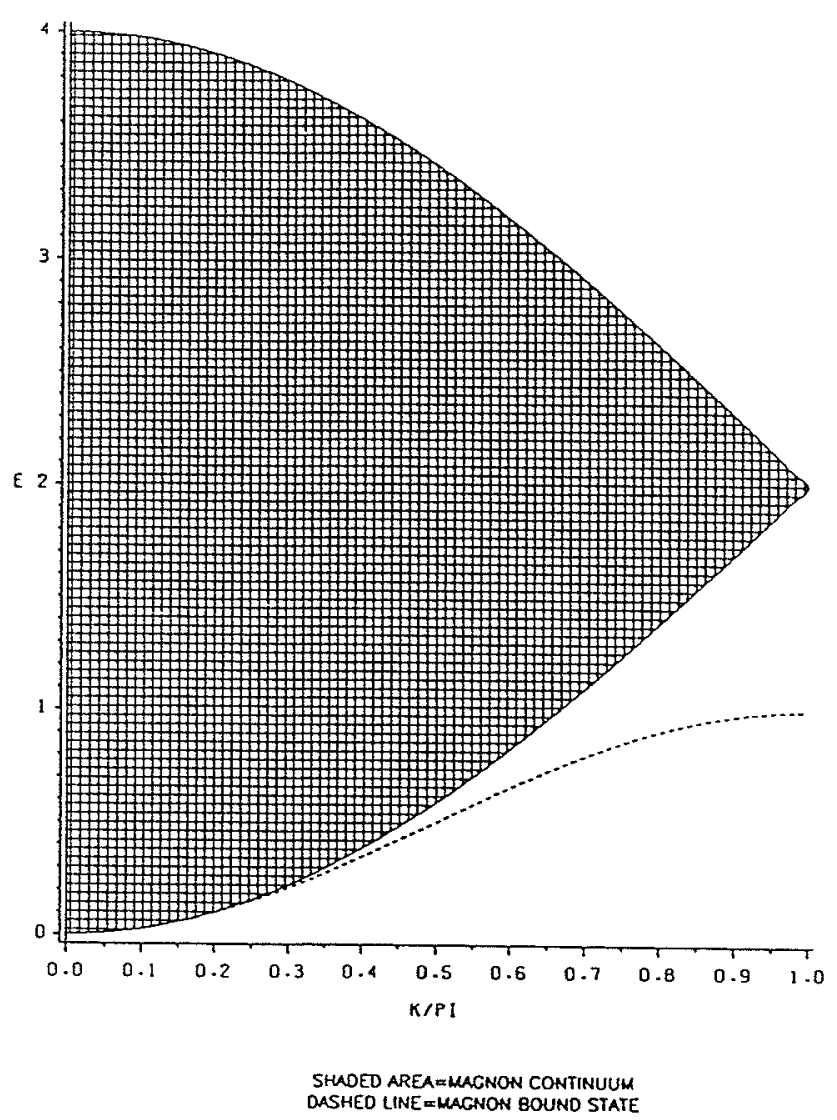

Figure 1.1: Isotropic Two-magnon Dispersion relation

neighbour interaction) case it is determined by,

$$
\Omega(\vec{K}, \vec{k})=8 S J\left(1-\cos \frac{K a}{2} \cos k a\right)
$$

Historically it was Bethe [12] who first discovered the bound complexes of spin waves in the $1 \mathrm{D}$ ferromagnet, and, which led to general concern about the spectra of two interacting magnons in higher dimensions. Dyson [13] derived a weak $T^{4}$ correction to its temperature effect on the magnetization. Wortis [14] established the 
existence of two-magnon bound states in $2 \mathrm{D}$ and $3 \mathrm{D}$ ferromagnets. Various extensions have been made by other workers, especially for the incorporation of uniaxial anisotropy[15],[16] and of biquadratic exchange [17],[18]. Callaway[19] showed that the doublet bound state broadened into a resonance inside the continuum, while Loly and Choudhury[20] investigated the bound-state-resonance mode for all $\vec{K}$.

The relationship between continuum structure and the occurrence of bound states, the role of van Hove singularities in determining the positions of resonances, and interpretation of corresponding Raman scattering experiments have attracted the interests of Loly and his collaborators[21]. Most of information has been obtained for the nearest-neighbour(NN) simple-cubic(SC) Heisenberg ferromagnet with Ising and uniaxial anisotropies. The SC Heisenberg ferromagnet is discussed in [22] with next-nearest neighbour (NNN) exchange interaction, where it was shown that the parameters could be chosen to prevent the formation of bound states. The NN SC Heisenberg model with various values of uniaxial anisotropy was studied in [23]. The $1 \mathrm{D}$ chain and square lattice are investigated in [8] and [24] respectively. This thesis studies the rather more complicated 2-D triangular ferromagnet. This has relevance to $\mathrm{FeBr}_{2}[25]$ and possibly to nuclear magnetism in layers of ${ }^{3} \mathrm{He}$ on graphite[26]. Wada, Ishikawa and Oguchi [27] discussed the bound states in these triangular ferromagnets for spin $1 / 2$ and Tonegawa[15] also did some relevant work.

In this Chapter we have given a brief outline of the history of magnetism before discussing the basic concepts such as the Heisenberg Hamiltonian, spin wave, magnon and magnon-magnon interaction. It is hoped that this may serve as an useful refer- 
ence for students who just begin the study of two-magnon problem. Chapter 2 first discusses the nature and approaches to the two-magnon problem. Then the timedependent Green function is introduced. Section 2.3 displays the derivation of the Dyson equation for the two-magnon propagators at $T=0 K$ in full detail. It is then applied to the triangular ferromagnet in Section 3.1. Critical points (van Hove type singularities) and Lattice Green functions for the non-interacting two-magnon problem are studied in section 3.2 and section 3.3 respectively. Chapter 4 discusses the results of numerical computations for bound states and resonances in non-anisotropic and anisotropic situation. A comparison with the Raman scattering experiments on $\mathrm{FeBr}_{2}$, which has layers of triangular ferromagnetic sheets is attempted, as a step towards a more complete analysis of the actual anisotropic $3 D$ problem. The conclusions are given in Chapter 5. 


\section{Chapter 2}

\section{The Two-magnon Problem and the Green Function Method}

In this chapter we will discuss the description of two-magnon problem, introduce double-time Green's function and derive the general Dyson's equation.

\subsection{Description of Two-magnon States}

An undisturbed two-magnon state $\left|\vec{k}_{1}, \vec{k}_{2}\right\rangle$, a system containing two noninteracting magnons with wavevectors $\vec{k}_{1}, \vec{k}_{2}$, is created by applying the magnon creation operator $a_{\vec{k}_{1}}^{+} a_{\vec{k}_{2}}^{+}$as defined in eq. 1.7.

When $\vec{k}_{1} \neq \vec{k}_{2}$,

$$
\begin{aligned}
\left|\vec{k}_{1}, \vec{k}_{2}\right\rangle & =\frac{1}{N} \sum_{i j} \exp \left(i \vec{k}_{1} \cdot \vec{r}_{i}+i \vec{k}_{2} \cdot r_{j}\right) a_{i}^{+} a_{j}^{+} \mid 0> \\
& =\frac{1}{N} \sum_{i j} \exp \left(i \vec{k}_{1} \cdot \vec{r}_{i}+i \vec{k}_{2} \cdot r_{j}\right)\left(1+(\sqrt{2}-1) \delta_{i j}\right) \mid i j>
\end{aligned}
$$

Here remember that $a_{i}^{+}|0\rangle=|i\rangle$, but $a_{i}^{+}|i\rangle=\sqrt{2}|i i\rangle$. 
When $\vec{k}_{1}=k_{2}$

$$
\begin{aligned}
\left|\vec{k}_{1}, \vec{k}_{1}\right\rangle & =\frac{1}{\sqrt{2}} a_{\vec{k}_{1}}^{+} a_{\vec{k}_{1}}^{+} \mid 0> \\
& =\frac{1}{\sqrt{2}} \frac{1}{N} \sum_{i j} \exp \left[i \vec{k}_{1} \cdot\left(r_{i}+r_{j}\right)\right]\left[1+(\sqrt{2}-1) \delta_{i j}\right] \mid i j>
\end{aligned}
$$

For a special case $S=1 / 2$, the state $\mid i i>$ is not allowed,

$$
\begin{aligned}
& \mid \vec{k}_{1}, \vec{k}_{2}>_{\left(\vec{k}_{1} \neq \vec{k}_{2}\right)}=\frac{1}{N} \sum_{i j} \exp \left(i \vec{k}_{1} \cdot \vec{r}_{i}+i \vec{k}_{2} \cdot r_{j}\right)\left(1-\delta_{i j}\right) \mid i j> \\
&\left|\vec{k}_{1}, \vec{k}_{2}>_{\left(\vec{k}_{1}=\vec{k}_{2}\right)}=\frac{1}{\sqrt{2}} \frac{1}{N} \sum_{i j} \exp \left[i \vec{k}_{1} \cdot\left(r_{i}+r_{j}\right)\right]\left[1-\delta_{i j}\right]\right| i j>
\end{aligned}
$$

The total energy is given in terms of $\left(\vec{k}_{1}, \vec{k}_{2}\right)$ by eq. 1.9 and in terms of $(\vec{K}, \vec{k})$ by eq. 1.10 .

It is known that such a non-interacting two-magnon state is not an energy eigenstate of Heisenberg Hamiltonian because of the scattering between magnons as we see from eq. (1.8).

There are several different approaches to solve the two-magnon energy eigenstate problem. However the problem itself must first be well defined before our discussing the details of these approaches.

- A group of states $\{\mid i j>\}^{\prime} s,(i=1, \cdots, N)$ forms a complete orthonormal subspace so that the Heisenberg Hamiltonian has no matrix elements connecting these states with any state outside the subspace (for example, $\langle l|H| i j\rangle=<$ $\left.0\left|a_{l}^{-} H a_{i}^{+} a_{j}^{+}\right| 0>=0\right)$.

- Two-magnon energy eigenstates are those solutions of Schrödinger equation 
$H|\phi>=E| \phi>$, which can be exactly expanded in above subspace, i.e.

$$
\left|\phi>=\sum_{i j} f_{i j}\right| i j>
$$

- Two-magnon H-eigenstates can also be well described in a reciprocal subspace $\left\{\left|\vec{k}_{1}, \vec{k}_{2}\right\rangle\right\}$ 's, where $\vec{k}_{1}, \vec{k}_{2}$ are restricted in 1-st Brillouin zone, i.e.

$$
\left|\phi>=\sum_{\vec{k}_{1}, \vec{k}_{2}} f\left(\vec{k}_{1}, \vec{k}_{2}\right)\right| \vec{k}_{1}, \vec{k}_{2}>
$$

- It can be further proved that the total wavevector $\vec{K}=\vec{k}_{1}+\vec{k}_{2}$ is conserved in two-magnon scattering, in other words, solutions belonging to distinct values of $\vec{K}$ are orthogonal, $<\vec{k}_{1}^{\prime}, \vec{k}_{2}^{\prime}|H| \vec{k}_{1}, \vec{k}_{2}>\propto \delta\left(\vec{k}_{1}+\vec{k}_{2}=\vec{k}_{1}^{\prime}+\vec{k}_{2}^{\prime}\right)$. So that,

$$
\left|\phi>=\sum_{\vec{k}_{1}+\vec{k}_{2}=\vec{K}} f\left(\vec{k}_{1}, \vec{k}_{2}\right)\right| \vec{k}_{1}, \vec{k}_{2}>
$$

While the energy eigenstate $\mid \phi>$ may be denoted by $\mid E, \vec{K}>$.

Let us find the relationship between the coefficients in real space $-f_{i j}$ and in reciprocal space- $f\left(\vec{k}_{1}, \vec{k}_{2}\right)$. Neglecting some normalization factors we have,

$$
\begin{aligned}
\mid E, \vec{K}> & =\sum_{\vec{k}_{1}+\vec{k}_{2}=\vec{K}} f\left(\vec{k}_{1}, \vec{k}_{2}\right)\left|\vec{k}_{1}, \vec{k}_{2}\right\rangle \\
& =\frac{1}{N} \sum_{\vec{k}_{1}+\vec{k}_{2}=\vec{K}} \sum_{i j} f\left(\vec{k}_{1}, \vec{k}_{2}\right) e^{i \vec{k}_{1} \cdot \vec{r}_{i}} e^{i \vec{k}_{2} \cdot \vec{r}_{j}} \mid i j>
\end{aligned}
$$

Replacing $\vec{k}_{1}, \vec{k}_{2}$ by total and relative wavevectors $\vec{K}, \vec{k}$ (eq. 1.11 ) and defining,

$$
\vec{R}_{i j}=\left(r_{i}+r_{j}\right) / 2, \quad \vec{r}_{i j}=r_{i}-r_{j}
$$


We have,

$$
\begin{aligned}
\mid E, \vec{K}> & =\sum_{i j} e^{i \vec{K} \cdot \vec{R}_{i j}}\left(\frac{1}{N} \sum_{\vec{k}} f(\vec{K}, \vec{k}) e^{i \vec{k} \cdot \vec{r}_{i j}}\right) \mid i j> \\
& =\sum_{i j} e^{i \vec{K} \cdot \vec{R}_{i j}} F\left(r_{i j}\right) \mid i j>
\end{aligned}
$$

This is the eq. 1.12 given in chapter 1 . Clearly $F\left(r_{i j}\right)$ is connected with the $\vec{k}$-space counterpart $f(\vec{K}, \vec{k})$ by a Fourier transformation,

$$
F(r)=\sum_{\vec{k}} e^{i \vec{k} \cdot \vec{r}} f(\vec{K}, \vec{k})
$$

Various methods have been developed to work out these coefficients so that the twomagnon energy eigenstate of which some, e.g. Bethe Ansatz [12] and real space rescaling [28] are really only useful in one-dimension. The Green function method is useful in any dimension, such as $2 \mathrm{D}$ triangular lattice studied in this thesis.

\subsection{Double-time Green Functions}

We shall give a brief account of the basic properties of the double-time Green function[29], and explore its application to our two-magnon problem. Generally, correlation functions $F_{B A}\left(t, t^{\prime}\right), F_{A B}\left(t, t^{\prime}\right)$ are defined by a thermodynamic ensemble average,

$$
\begin{aligned}
& F_{B A}=\left\langle B\left(t^{\prime}\right) A(t)\right\rangle \\
& F_{A B}=\left\langle A(t) B\left(t^{\prime}\right)\right\rangle
\end{aligned}
$$

Where the operators are defined in Heisenberg representation,

$$
A(t)=e^{i H t} A e^{-i H t}
$$


We further define spectral intensity $J(\omega)$ and let,

$$
\begin{aligned}
& F_{A B}=\int_{-\infty}^{\infty} J(\omega) e^{\frac{\omega}{\theta}} e^{-i \omega\left(t-t^{\prime}\right)} d \omega \\
& F_{B A}=\int_{-\infty}^{\infty} J(\omega) e^{-i \omega\left(t-t^{\prime}\right)} d \omega
\end{aligned}
$$

Where $\theta$ is temperature. Denoting the energy eigenstates of system by $|\mu\rangle,|\gamma\rangle$, and the partition function by $Q$, we have,

$$
J(\omega)=Q^{-1} \sum_{\mu, \gamma}<\gamma|A| \mu><\mu|B| \gamma>e^{\frac{\omega}{\theta}} \delta\left(E_{\mu}-E_{\gamma}-\omega\right)
$$

Retarded and advanced double-time Green functions are introduced to solve many problems which are associated with the correlation functions,

$$
\begin{aligned}
& \left.G_{r}\left(t, t^{\prime}\right)=\ll A(t) ; B\left(t^{\prime}\right) \gg_{r}=-i \theta\left(t-t^{\prime}\right)<\left[A(t), B\left(t^{\prime}\right)\right]\right\rangle \\
& \left.G_{a}\left(t, t^{\prime}\right)=\ll A(t) ; B\left(t^{\prime}\right) \gg_{a}=i \theta\left(t^{\prime}-t\right)<\left[A(t), B\left(t^{\prime}\right)\right]\right\rangle
\end{aligned}
$$

Where $\theta(t)$ is the usual Heaviside step function,

$$
\theta(t)= \begin{cases}1 & t>0 \\ 0 & t<0\end{cases}
$$

Recalling the Heisenberg equation of motion,

$$
i \frac{d A}{d t}=A H-H A
$$

an equation of motion for the Green function can be derived,

$$
i \frac{d G_{r, a}}{d t}=\delta\left(t-t^{\prime}\right)<\left[A(t) ; B\left(t^{\prime}\right)\right]>+\ll[A(t) ; H(t)] ; B\left(t^{\prime}\right) \gg_{r, a}
$$


Let $G_{r}(\omega)$, which is sometimes also denoted as $\ll A(t) ; B\left(t^{\prime}\right) \gg_{\omega}$ be the Fourier component of $G_{r}\left(t-t^{\prime}\right)$,

$$
G_{r}(\omega)=\frac{1}{2 \pi} \int_{-\infty}^{+\infty} G_{\tau}(t) e^{i E t} d t
$$

Then,

$$
\omega G_{\tau}(\omega)=\langle[A, B]\rangle+\ll[A ; H] ; B \gg \omega
$$

We note that we obtained a chain of coupled equations for the Green functions. One can usually uncouple the equations by some kind of approximate methods, to reduce it to a finite set of equations, which can then be solved.

Substitute eq. (2.2) and eq. (2.4) into eq. (2.7), we have,

$$
\begin{aligned}
G_{r, a}(E) & =\int_{-\infty}^{\infty} d \omega J(\omega)\left(e^{\frac{\omega}{\theta}}-1\right) \frac{1}{2 \pi i} \int_{-\infty}^{\infty} d t e^{i(E-\omega) t} \theta(t) \\
& =\frac{1}{2 \pi} \int_{-\infty}^{\infty}\left(e^{\frac{\omega}{\theta}}-1\right) J(\omega) \frac{d \omega}{E-\omega \pm i \varepsilon} \quad(\varepsilon \rightarrow 0)
\end{aligned}
$$

Here we established the connection between the Green function and the spectral intensity, i.e.

$$
J(\omega)=\frac{2}{\left(e^{\frac{\omega}{\theta}}-1\right)} \operatorname{Im} G_{r}(\omega)
$$

and,

$$
\operatorname{Re} G_{r}(E)=\frac{1}{\pi} \mathbf{P} \int_{-\infty}^{\infty} \frac{\operatorname{Im} G_{r}(\omega)}{\omega-E} d \omega
$$

Taking advantage of the above well-established formulae we could define a twomagnon Green function,

$$
\begin{aligned}
G\left(\vec{k}_{1}, \vec{k}_{2} ; \vec{k}_{1}^{\prime}, \vec{k}_{2}^{\prime}, t\right) & =-i \theta(t)<0\left|\left[S_{\vec{k}_{1}}^{-}(t) S_{\vec{k}_{2}}^{-}(t) ; S_{\vec{k}_{1}^{\prime}}^{+}(0) S_{\vec{k}_{2}^{\prime}}^{+}(0)\right]\right| 0> \\
& =\ll S_{\vec{k}_{1}}^{-}(t) S_{\vec{k}_{2}}^{-}(t) \mid S_{\vec{k}_{1}^{\prime}}^{+}(0) S_{\vec{k}_{2}^{\prime}}^{+}(0) \gg
\end{aligned}
$$


It is different from the above general form by the fact that it only calculates the expectation value in the ground state instead of the thermodynamic average of ensemble.

The equation of motion for the Green's function has the standard form

$$
\omega G\left(\vec{k}_{1}, \vec{k}_{2} ; \vec{k}_{1}^{\prime}, \vec{k}_{2}^{\prime}, \omega\right)=<0\left|\left[S_{\vec{k}_{1}}^{-} S_{\vec{k}_{2}}^{-} ; S_{\vec{k}_{1}^{\prime}}^{+} S_{\vec{k}_{2}^{\prime}}^{+}\right]\right| 0>+\ll\left[S_{\vec{k}_{1}}^{-} S_{\vec{k}_{2}}^{-} ; H\right] \mid S_{\vec{k}_{1}^{\prime}}^{+} S_{\vec{k}_{2}^{\prime}}^{+} \gg_{\omega}
$$

Since,

$$
S_{\vec{k}_{1}}^{+} S_{\vec{k}_{2}}^{+}|0>=| \vec{k}_{1}, \vec{k}_{2}>\quad \text { (neglecting normalization) }
$$

The so-called correlation function,

$$
<0\left|S_{\vec{k}_{1}}^{-}(t) S_{\vec{k}_{2}}^{-}(t) S_{\vec{k}_{1}^{\prime}}^{+}(0) S_{\vec{k}_{2}^{\prime}}^{+}(0)\right| 0>=\sum_{E, \vec{K}}<\vec{k}_{1}, \vec{k}_{2}|E, \vec{K}><E, \vec{K}| \vec{k}_{1}^{\prime}, \vec{k}_{2}^{\prime}>e^{i\left(E-E_{0}\right) t}
$$

Therefore from equation (2.2),

$$
\begin{aligned}
J_{\vec{K}}(\omega) & =\sum_{E, \vec{K}}<\vec{k}_{1}, \vec{k}_{2}|E, \vec{K}><E, \vec{K}| \vec{k}_{1}^{\prime}, \vec{k}_{2}^{\prime}>\delta\left(E-E_{0}-\omega\right) \\
& =<\vec{k}_{1}, \vec{k}_{2}|\omega, \vec{K}><\omega, \vec{K}| \vec{k}_{1}^{\prime}, \vec{k}_{2}^{\prime}> \\
& =f_{\omega, \vec{K}}\left(\vec{k}_{1}, \vec{k}_{2}\right) f_{\omega, \vec{K}}^{*}\left(\vec{k}_{1}^{\prime}, \vec{k}_{2}^{\prime}\right)
\end{aligned}
$$

In terms of total wavefunction (let $\vec{K}=\vec{K}^{\prime}$ ) and relative wavevectors $\left(\vec{k}, \vec{k}^{\prime}\right)$ (eq. 1.11), we get,

$$
\operatorname{Im} G\left(\vec{K}, \vec{k}, \vec{k}^{\prime}, \omega\right) \propto f_{\omega, \vec{K}}\left(\vec{k}_{1}, \vec{k}_{2}\right) f_{\omega, \vec{K}}^{*}\left(\vec{k}_{1}^{\prime}, \vec{k}_{2}^{\prime}\right)
$$

We now introduce a partial Fourier transformation,

$$
G(i j, \vec{K}, \omega)=\frac{1}{N} \sum_{\vec{k} \vec{k}^{\prime}} G\left(\vec{K} \vec{k}, \vec{K} \vec{k}^{\prime}, \omega\right) \exp \left(-i \vec{k} \cdot \vec{R}_{i}+i \vec{k}^{\prime} \cdot \vec{R}_{j}\right)
$$


Clearly the real space coefficient of a two-magnon state is then given by,

$$
\operatorname{Im} G(i, j, \vec{K}, \omega) \propto F_{\omega, \vec{K}}\left(\vec{R}_{i}\right) F_{\omega, \vec{K}}^{*}\left(\vec{R}_{j}\right)
$$

And,

$$
\left|F_{\omega, \vec{K}}\left(\vec{R}_{l}, \vec{R}_{l}\right)\right|^{2} \propto \operatorname{Im} G(l, l, \vec{K}, \omega)
$$

The imaginary part of Green function $G(l, l, \vec{K}, \omega)$ represents just what we desired, the relative amplitude of the projection of a two-magnon state on the baseket $|i j\rangle$ (where, $\vec{R}_{i}-\vec{R}_{j}=\vec{R}_{l}$ ), in other words the probability of finding two spin deviations created with a separation $\vec{R}_{l}$ in the two-magnon energy eigenstate $|E, \vec{K}\rangle$ of the Heisenberg Hamiltonian.

\subsection{Dyson's Equation of the Two-magnon Prop- agator}

To study the two-magnon spectrum we need to carry out the procedure described in section 2.2 (from eq. 2.11) to eq. 2.15). Here the ground state is taken as $S_{z} \mid 0>=$ $-S \mid 0>$, and the Heisenberg Hamiltonian in the presence of Ising and single-ion anisotropies is given by equation (1.2).

Let us first evaluate the two commutators in eq. (2.12).

The first one,

$$
\begin{aligned}
{\left[S_{\vec{k}_{1}}^{-} S_{\vec{k}_{2}}^{-} ; S_{\vec{k}_{1}^{\prime}}^{+} S_{\vec{k}_{2}^{\prime}}^{+}\right]=} & \frac{1}{N^{2}} \sum_{i j i^{\prime} j^{\prime}} \exp \left(-i \vec{k}_{1} \cdot \vec{r}_{i}-i \vec{k}_{2} \cdot \vec{r}_{j}+i \vec{k}_{1}^{\prime} \cdot \vec{r}_{i^{\prime}}+i \vec{k}_{2}^{\prime} \cdot \vec{r}_{j^{\prime}}\right) \cdot\left[S_{i}^{-} S_{j}^{-}, S_{i^{\prime}}^{+} S_{j^{\prime}}^{+}\right] \\
= & \frac{1}{N^{2}} \sum_{i j i^{\prime} j^{\prime}} \exp \left(-i \vec{k}_{1} \cdot \vec{r}_{i}-i \vec{k}_{2} \cdot \vec{r}_{j}+i \vec{k}_{1}^{\prime} \cdot \vec{r}_{i^{\prime}}+i \vec{k}_{2}^{\prime} \cdot \vec{r}_{j^{\prime}}\right) \\
& \cdot(-2)\left(S_{i}^{-} S_{i^{\prime}}^{+} S_{j}^{z} \delta_{j j^{\prime}}+S_{i}^{-} S_{j}^{z} S_{j^{\prime}}^{+} \delta_{j i^{\prime}}+S_{i^{\prime}}^{+} S_{i}^{z} S_{j}^{-} \delta_{i j^{\prime}}+S_{i}^{z} S_{j^{\prime}}^{+} S_{j}^{-} \delta_{i i^{\prime}}\right)
\end{aligned}
$$




$$
\begin{aligned}
<0\left|\left[S_{\vec{k}_{1}}^{-} S_{\vec{k}_{2}}^{-} ; S_{\vec{k}_{1}^{\prime}}^{+} S_{\vec{k}_{2}^{\prime}}^{+}\right]\right| 0> \\
=\frac{2 S}{N} \delta\left(\vec{k}_{1}^{\prime}, \vec{k}_{2}\right)<0\left|\frac{1}{N} \sum_{i i^{\prime}} \exp \left(-i \vec{k}_{1} \cdot \vec{r}_{i}+i \vec{k}_{1}^{\prime} \cdot \vec{r}_{i^{\prime}}\right) S_{i}^{-} S_{i^{\prime}}^{+}\right| 0> \\
\quad+\sum_{i i^{\prime} j} \frac{2(2 S)}{N}\left(-S+\delta_{i j}\right) \exp \left[-i\left(\vec{k}_{2}-\vec{k}_{1}^{\prime}\right) \cdot \vec{r}_{i^{\prime}}\right] \exp \left[-i\left(\vec{k}_{1}-\vec{k}_{2}^{\prime}\right) \cdot \vec{r}_{i}\right] \\
=4 S^{2} \delta\left(\vec{k}_{1}, \vec{k}_{1}^{\prime}\right) \delta\left(\vec{k}_{2}, \vec{k}_{2}^{\prime}\right)+4 S^{2} \delta\left(\vec{k}_{2}, \vec{k}_{1}^{\prime}\right) \delta\left(\vec{k}_{1}, \vec{k}_{2}^{\prime}\right)-\frac{4 S}{N} \delta\left(\vec{k}_{1}+\vec{k}_{2}, \vec{k}_{1}^{\prime}+\vec{k}_{2}^{\prime}\right)
\end{aligned}
$$

The second one,

$$
\begin{aligned}
{\left[S_{\vec{k}_{1}}^{-} S_{\overrightarrow{k_{2}}}^{-} ; H\right]=} & \frac{1}{N} \sum_{i j} \exp \left(-i \vec{k}_{1} \cdot \vec{r}_{i}-i \vec{k}_{2} \cdot \vec{r}_{j}\right) \cdot\left[S_{i}^{-} S_{j}^{-} ; H\right] \\
= & -\frac{1}{N} \sum_{i j i^{\prime} j^{\prime}} I_{i^{\prime} j^{\prime}} \exp \left(-i \vec{k}_{1} \cdot \vec{r}_{i}-i \vec{k}_{2} \cdot \vec{r}_{j}\right) \cdot \underbrace{\left[S_{i}^{-} S_{j}^{-} ; S_{i^{\prime}}^{z} S_{j^{\prime}}^{z}\right]}_{(a)} \\
& -\frac{1}{N} \sum_{i j i^{\prime} j^{\prime}} J_{i^{\prime} j^{\prime}} \exp \left(-i \vec{k}_{1} \cdot \vec{r}_{i}-i \vec{k}_{2} \cdot \vec{r}_{j}\right) \cdot \underbrace{\left[S_{i}^{-} S_{j}^{-}, S_{i^{\prime}}^{+} S_{j^{\prime}}^{-}\right]}_{(b)} \\
& -\frac{1}{N} \sum_{i j^{\prime}} D \exp \left(-i \vec{k}_{1} \cdot \vec{r}_{i}-i \vec{k}_{2} \cdot \vec{r}_{j}\right) \cdot \underbrace{\left[S_{i}^{-} S_{j}^{-} ;\left(S_{i^{\prime}}^{z}\right)^{2}\right]}_{(c)}
\end{aligned}
$$

Where the term (a),

$$
\begin{aligned}
{\left[S_{i}^{-} S_{j}^{-} ; S_{i^{\prime}}^{z} S_{j^{\prime}}^{z}\right] } & =S_{i}^{-}\left[S_{j}^{-} ; S_{i^{\prime}}^{z} S_{j^{\prime}}^{z}\right]+\left[S_{i}^{-} ; S_{i^{\prime}}^{z} S_{j^{\prime}}^{z}\right] S_{j}^{-} \\
& =S_{i}^{-} S_{i^{\prime}}^{z}\left[S_{j}^{-} ; S_{j^{\prime}}^{z}\right]+S_{i}^{-}\left[S_{j}^{-} ; S_{i^{\prime}}^{z}\right] S_{j^{\prime}}^{z}+S_{i^{\prime}}^{z}\left[S_{i}^{-} ; S_{j^{\prime}}^{z}\right] S_{j}^{-}+\left[S_{i}^{-} ; S_{i^{\prime}}^{z}\right] S_{j^{\prime}}^{z} S_{j}^{-} \\
& =S_{i}^{-} S_{i^{\prime}}^{z} S_{j}^{-} \delta_{j j^{\prime}}+S_{i}^{-} S_{j}^{-} S_{j^{\prime}}^{z} \delta_{j i^{\prime}}+S_{i^{\prime}}^{z} S_{i}^{-} S_{j}^{-} \delta_{i j^{\prime}}+S_{i}^{-} S_{j^{\prime}}^{z} S_{j}^{-} \delta_{i i^{\prime}}
\end{aligned}
$$

The term (b)

$$
\begin{aligned}
{\left[S_{i}^{-} S_{j}^{-} ; S_{i^{\prime}}^{+} S_{j^{\prime}}^{-}\right] } & =\left[S_{i}^{-} S_{j}^{-} ; S_{i^{\prime}}^{+}\right] S_{j^{\prime}}^{-} \\
& =S_{i}^{-}\left[S_{j}^{-} ; S_{i^{\prime}}^{+}\right] S_{j^{\prime}}^{-}+\left[S_{i}^{-} ; S_{i^{\prime}}^{+}\right] S_{j}^{-} S_{j^{\prime}}^{-} \\
& =-2 S_{i}^{-} S_{j}^{z} S_{j^{\prime}}^{-} \delta_{j i^{\prime}}-2 S_{i}^{z} S_{j}^{-} S_{j^{\prime}}^{-} \delta_{i i^{\prime}}
\end{aligned}
$$


The term (c)

$$
\left[S_{i}^{-} S_{j}^{-} ;\left(S_{i^{\prime}}^{z}\right)^{2}\right]=S_{i}^{-}\left(S_{j}^{z} S_{j}^{-}+S_{j}^{-} S_{j}^{z}\right) \cdot \delta_{j i^{\prime}}+\left(S_{i}^{z} S_{i}^{-}+S_{i}^{-} S_{i}^{z}\right) S_{j}^{-} \cdot \delta_{i i^{\prime}}
$$

With appropriate relabelling we have,

$$
\begin{aligned}
& {\left[S_{\vec{k}_{1}}^{-} S_{\vec{k}_{2}}^{-}, H\right]=-\frac{1}{N} \sum_{i i^{\prime} j} I_{i^{\prime} j} \exp \left(-i \vec{k}_{1} \cdot \vec{r}_{i}-i \vec{k}_{2} \cdot \vec{r}_{j}\right) S_{i}^{-} S_{i^{\prime}}^{z} S_{j}^{-} \quad \ldots 1} \\
& -\frac{1}{N} \sum_{i i^{\prime} j} I_{j i^{\prime}} \exp \left(-i \vec{k}_{1} \cdot \vec{r}_{i}-i \vec{k}_{2} \cdot \vec{r}_{j}\right) S_{i}^{-} S_{j}^{-} S_{i^{\prime}}^{z} \quad \ldots 2 \\
& -\frac{1}{N} \sum_{i i^{\prime} j} I_{i^{\prime} i} \exp \left(-i \vec{k}_{1} \cdot \vec{r}_{i}-i \vec{k}_{2} \cdot \vec{r}_{j}\right) S_{i^{\prime}}^{z} S_{i}^{-} S_{j}^{-} \quad \ldots 3 \\
& -\frac{1}{N} \sum_{i i^{\prime} j} I_{i i^{\prime}} \exp \left(-i \vec{k}_{1} \cdot \vec{r}_{i}-i \vec{k}_{2} \cdot \vec{r}_{j}\right) S_{i}^{-} S_{i^{\prime}}^{z} S_{j}^{-} \quad \ldots 4 \\
& +\frac{2}{N} \sum_{i i^{\prime} j} J_{j i^{\prime}} \exp \left(-i \vec{k}_{1} \cdot \vec{r}_{i}-i \vec{k}_{2} \cdot \vec{r}_{j}\right) S_{i}^{-} S_{j}^{z} S_{i^{\prime}}^{-} \quad \ldots 5 \\
& +\frac{2}{N} \sum_{i i^{\prime} j} J_{i i^{\prime}} \exp \left(-i \vec{k}_{1} \cdot \vec{r}_{i}-i \vec{k}_{2} \cdot \vec{r}_{j}\right) S_{i}^{z} S_{j}^{-} S_{i^{\prime}}^{-} \quad \ldots 6 \\
& -\frac{D}{N} \sum_{i j} \exp \left(-i \vec{k}_{1} \cdot \vec{r}_{i}-i \vec{k}_{2} \cdot \vec{r}_{j}\right) S_{i}^{-}\left(S_{j}^{z} S_{j}^{-}+S_{j}^{-} S_{j}^{z}\right) \quad \ldots 7 \\
& -\frac{D}{N} \sum_{i j} \exp \left(-i \vec{k}_{1} \cdot \vec{r}_{i}-i \vec{k}_{2} \cdot \vec{r}_{j}\right)\left(S_{i}^{z} S_{i}^{-}+S_{i}^{-} S_{i}^{z}\right) S_{j}^{-} \quad \ldots 8
\end{aligned}
$$

Note that Green's functions with our definition can be also written as

$$
\ll A(t) ; B(0) \gg_{\omega}=\frac{1}{2 \pi} \sum_{\mu}\left(\frac{\langle 0|A| \mu><\mu|B| 0\rangle}{\omega-\omega_{\mu}+\omega_{0}}-\frac{\langle 0|B| \mu><\mu|A| 0\rangle}{\omega+\omega_{\mu}-\omega_{0}}\right)
$$

And since, $<0\left|S^{+} \longrightarrow 0, S^{-}\right| 0>0$, so:

$$
\ll\left[S_{\vec{k}_{1}}^{-} S_{\vec{k}_{2}}^{-}, H\right] \mid S_{\vec{k}_{1}^{\prime}}^{+} S_{\vec{k}_{2}^{\prime}}^{+} \gg=\frac{1}{2 \pi} \sum_{\mu} \frac{<0\left|\left[S_{\vec{k}_{1}}^{-} S_{\vec{k}_{2}}^{-}, H\right]\right| \mu><\mu\left|S_{\vec{k}_{1}^{\prime}}^{+} S_{\vec{k}_{2}^{\prime}}^{+}\right| 0>}{\omega-\omega_{\mu}+\omega_{0}}
$$


We evaluate the terms in eq. (2.16) respectively.

For term 1, note that $S_{i}^{-} S_{i^{\prime}}^{z} S_{j}^{-}=S_{i^{\prime}}^{z} S_{i}^{-} S_{j}^{-}+S_{i}^{-} S_{j}^{-} \delta_{i i^{\prime}}$, we have,

$$
\begin{aligned}
- & \frac{1}{N} \sum_{i i^{\prime} j} I_{i^{\prime} j} \exp \left(-i \vec{k}_{1} \cdot \vec{r}_{i}-i \vec{k}_{2} \cdot \vec{r}_{j}\right) \ll S_{i}^{-} S_{i^{\prime}}^{z} S_{j}^{-} \mid S_{\vec{k}_{1}^{\prime}}^{+} S_{\vec{k}_{2}^{\prime}}^{+} \gg \\
= & -\frac{1}{N} \sum_{i i^{\prime} j} I_{i^{\prime} j} \exp \left(-i \vec{k}_{1} \cdot \vec{r}_{i}-i \vec{k}_{2} \cdot \vec{r}_{j}\right)(-S) \sum_{\mu} \frac{\left\langle 0\left|S_{i}^{-} S_{j}^{-}\right| \mu><\mu\left|S_{\vec{k}_{1}^{\prime}}^{+} S_{\vec{k}_{2}^{\prime}}^{+}\right| 0\right\rangle}{\omega-\omega_{\mu}+\omega_{0}} \\
& \quad-\frac{1}{N} \sum_{i j} I_{i j} \exp \left(-i \vec{k}_{1} \cdot \vec{r}_{i}-i \vec{k}_{2} \cdot \vec{r}_{j}\right) \sum_{\mu} \frac{\left.<0\left|S_{i}^{-} S_{j}^{-}\right| \mu><\mu\left|S_{\vec{k}_{1}^{\prime}}^{+} S_{\vec{k}_{2}^{\prime}}^{+}\right| 0\right\rangle}{\omega-\omega_{\mu}+\omega_{0}} \\
= & S \cdot I(0) G\left(\vec{k}_{1}, \vec{k}_{2} ; \vec{k}_{1}^{\prime}, \vec{k}_{2}^{\prime}, \omega\right)-\frac{1}{N} \sum_{\vec{q}} I(\vec{q}) G\left(\vec{k}_{1}+\vec{q}, \vec{k}_{2}-\vec{q} ; \vec{k}_{1}^{\prime}, \vec{k}_{2}^{\prime}, \omega\right)
\end{aligned}
$$

Here we transformed $I_{i^{\prime} j}$ to $I(\vec{q})$ (see eq. 1.6)

For term $\mathscr{2}$, note that $S_{i}^{-} S_{j}^{-} S_{i^{\prime}}^{z}=S_{i^{\prime}}^{z} S_{i}^{-} S_{j}^{-}+S_{i}^{-} S_{j}^{-}\left(\delta_{i i^{\prime}}+\delta_{j i^{\prime}}\right)$. Eventually it leads to the same result as term 1 .

If we treat the other terms with same strategy, we can obtain the following equation of motion for the $\vec{k}$-space Green's function,

$$
\begin{aligned}
\{\omega & \left.-2 S\left[2 I(0)-J\left(\frac{1}{2} \vec{K}+\vec{k}\right)-J\left(\frac{1}{2} \vec{K}-\vec{k}\right)\right]-2(2 S-1) D\right\} G\left(\vec{K}, \vec{k} ; \vec{K}^{\prime}, \vec{k}^{\prime} ; \omega\right) \\
= & 4 S^{2} \delta_{\vec{K}-\vec{K}^{\prime}}\left[\delta_{\vec{k}+\vec{k}^{\prime}}+\delta_{\vec{k}-\vec{k}^{\prime}}-\frac{1}{N S}\right] \\
& -\frac{2}{N} \sum_{\vec{q}}\left[I(\vec{q})-J\left(\frac{1}{2} \vec{K}-\vec{k}-\vec{q}\right)+D\right] G\left(\vec{K}, \vec{K}+\vec{q} ; \vec{K}^{\prime}, \vec{k}^{\prime} ; \omega\right)
\end{aligned}
$$

Where we have changed the variables to $\left(\vec{K}, \vec{k}, \vec{K}^{\prime}, \vec{k}^{\prime}\right)$, the total and relative wave vectors(see eq. 1.11).

Note that $2 S\left[2 I(0)-J\left(\frac{1}{2} \vec{K}+\vec{k}\right)-J\left(\frac{1}{2} \vec{K}-\vec{k}\right)\right]+2(2 S-1) D$ is just the total en- 
ergy of two non-interacting magnons(see eq.1.10). We could rewrite eq. (2.17) as:

$$
\begin{aligned}
G\left(\vec{K}, \vec{k} ; \vec{K}^{\prime}, \vec{k} ; \omega\right)= & \frac{4 S^{2}}{\omega-\Omega(\vec{K}, \vec{k})}\left\{\delta_{\vec{k}, \vec{k}^{\prime}}+\delta_{\vec{k},-\vec{k}^{\prime}}-\frac{1}{N S}\right\} \\
& -\frac{2}{N} \frac{1}{\omega-\Omega(\vec{K}, \vec{k})} \sum_{\vec{q}} I(\vec{q}) G\left(\vec{K}, \vec{k}+\vec{q} ; \vec{K}^{\prime}, \vec{k}^{\prime} ; \omega\right) \\
& -\frac{2 D}{N} \frac{1}{\omega-\Omega(\vec{K}, \vec{k})} \sum_{\vec{q}} G\left(\vec{K}, \vec{k}+\vec{q} ; \vec{K}^{\prime}, \vec{k}^{\prime} ; \omega\right) \\
& +\frac{2}{N} \frac{1}{\omega-\Omega(\vec{K}, \vec{k})} \sum_{\vec{q}} J\left(\frac{1}{2} \vec{K}-\vec{k}-\vec{q}\right) G\left(\vec{K}, \vec{k}+\vec{q} ; \vec{K}^{\prime}, \vec{k}^{\prime} ; \omega\right) \ldots[4]
\end{aligned}
$$

Letting $\vec{K}^{\prime}=\vec{K}$ (conservation of total momentum) and taking the partial Fourier transform(eq. 2.14), i.e. multipling eq. (2.18) by the factor $\exp \left(-i \vec{k} \cdot \vec{R}_{i}+i \vec{k}^{\prime} \cdot \vec{R}_{j}\right)$ and summing over the subscripts $\vec{k}, \vec{k}^{\prime}$, then the L.H.S. of eq. (2.18)

$$
G\left(\vec{K}, \vec{k} ; \vec{K}^{\prime}, \vec{k} ; \omega\right) \longrightarrow G(i, j, \vec{K}, \omega)
$$

On the R.H.S.,

$$
\begin{gathered}
{[1] \quad \longrightarrow \frac{1}{N} \sum_{\vec{k}, \vec{k}^{\prime}} e^{-i \vec{k} \cdot \vec{R}_{i}} e^{i \vec{k}^{\prime} \cdot \vec{R}_{j}} \frac{4 S^{2}}{\omega-\Omega(\vec{K}, \vec{k})}\left\{\delta_{\vec{k}, \vec{k}^{\prime}}+\delta_{\vec{k}_{,}-\vec{k}^{\prime}}-\frac{1}{N S}\right\}} \\
=\frac{4 S^{2}}{N} \sum_{\vec{k}} \frac{e^{-i \vec{k} \cdot \vec{R}_{i}}}{\omega-\Omega(\vec{K}, \vec{k})}\left\{2 \cos \left(\vec{k} \cdot \vec{R}_{j}\right)-\frac{1}{S} \delta_{j 0}\right\} \\
=\frac{8 S^{2}}{N} \sum_{\vec{k}} \frac{\cos \left(\vec{k} \cdot \vec{R}_{i}\right) \cos \left(\vec{k} \cdot \vec{R}_{j}\right)}{\omega-\Omega(\vec{K}, \vec{k})}\left(1-\frac{1}{2 S} \delta_{j 0}\right) \\
{[2] \longrightarrow \frac{1}{N} \sum_{\vec{k} \vec{k}^{\prime}} e^{-i \vec{k} \cdot \vec{R}_{i}} e^{i \vec{k}^{\prime} \cdot \vec{R}_{j}} \frac{1}{\omega-\Omega(\vec{K}, \vec{k})}\left(-\frac{2}{N}\right) \sum_{\vec{q}} I(\vec{q}) G\left(\vec{K}, \vec{k}+\vec{q} ; \vec{K}, \vec{k}^{\prime} ; \omega\right)} \\
=-\frac{2}{N^{2}} \sum_{\vec{k} \vec{k}^{\prime}} e^{-i \vec{k} \cdot \vec{R}_{i}} e^{i \vec{k}^{\prime} \cdot \vec{R}_{j}} \frac{1}{\omega-\Omega(\vec{K}, \vec{k})} \sum_{\vec{q}} I(\vec{q})\left[\sum_{l m} \frac{1}{N} e^{i(\vec{k}+\vec{q}) \cdot \vec{R}_{l}} e^{-i \vec{k}^{\prime} \cdot \vec{R}_{m}} G(l, m, \vec{K}, \omega)\right]
\end{gathered}
$$




$$
\begin{aligned}
= & -\frac{2}{N} \sum_{\vec{k}, m l} e^{i \vec{k} \cdot\left(\vec{R}_{l}-\vec{R}_{i}\right)}\left[\frac{1}{N} \sum_{\vec{k}^{\prime}} e^{-i \vec{k}^{\prime} \cdot\left(\vec{R}_{j}-\vec{R}_{m}\right)}\right] \\
& \cdot\left[\frac{1}{N} \sum_{\vec{q}} I(\vec{q}) e^{i \vec{q} \cdot \vec{R}_{l}}\right] \frac{1}{\omega-\Omega(\vec{K}, \vec{k})} G(l, m, \vec{K}, \omega) \\
=\quad & -\frac{2}{N} \sum_{\vec{k}, l} I(l) e^{i \vec{k} \cdot\left(\vec{R}_{l}-\vec{R}_{i}\right)} \frac{1}{\omega-\Omega(\vec{K}, \vec{k})} G(l, j, \vec{K}, \omega) \\
= & -2 \sum_{l} I(l)\left[\frac{1}{N} \sum_{\vec{k}} \frac{\cos \left(\vec{k} \cdot \vec{R}_{l}\right) \cos \left(\vec{k} \cdot \vec{R}_{i}\right)}{\omega-\Omega(\vec{K}, \vec{k})}\right] G(l, j, \vec{K}, \omega)
\end{aligned}
$$

$[3] \longrightarrow \frac{1}{N} \sum_{\vec{k} \vec{k}^{\prime}} e^{-i \vec{k} \cdot \vec{R}_{i}} e^{i \vec{k}^{\prime} \cdot \vec{R}_{j}}\left(-\frac{2 D}{N}\right) \frac{1}{\omega-\Omega(\vec{K}, \vec{k})} \sum_{\vec{q}} G\left(\vec{K}, \vec{k}+\vec{q} ; \vec{K}, \vec{k}^{\prime} ; \omega\right)$

$=\frac{1}{N} \sum_{\vec{k} \vec{k}^{\prime}} e^{-i \vec{k} \cdot \vec{R}_{i}} e^{i \vec{k}^{\prime} \cdot \vec{R}_{j}}\left(-\frac{2 D}{N}\right) \frac{1}{\omega-\Omega(\vec{K}, \vec{k})} \sum_{\vec{q}}\left[\frac{1}{N} \sum_{l m} e^{i(\vec{k}+\vec{q}) \cdot \vec{R}_{l}} e^{-i \vec{k}^{\prime} \cdot \vec{R}_{m}} G(l, m, \vec{K}, \omega)\right]$

$=-\sum_{\vec{k}, l m}\left(\frac{1}{N} \sum_{\vec{q}} e^{i \vec{q} \cdot \vec{R}_{l}}\right) \cdot\left[\frac{1}{N} \sum_{\vec{k}^{\prime}} e^{i \vec{k}^{\prime} \cdot\left(\vec{R}_{j}-\vec{R}_{m}\right)}\right] e^{i \vec{k} \cdot\left(\vec{R}_{l}-\vec{R}_{i}\right)}$

$$
\cdot \frac{2 D}{N} \frac{1}{\omega-\Omega(\vec{K}, \vec{k})} G(l, m, \vec{K}, \omega)
$$

$=-\frac{2 D}{N} \sum_{\vec{k}} \frac{e^{-i \vec{k} \cdot \vec{R}_{i}}}{\omega-\Omega(\vec{K}, \vec{k})} G(0, j, \vec{K}, \omega)$

$=-2 D \sum_{l}\left[\frac{1}{N} \sum_{\vec{k}} \frac{\cos \vec{k} \cdot \vec{R}_{l} \cos \vec{k} \cdot \vec{R}_{i}}{\omega-\Omega(\vec{K}, \vec{k})}\right] G(l, j, \vec{K}, \omega) \delta_{l, 0}$

$[4] \longrightarrow \frac{1}{N} \sum_{\vec{k} \vec{k}^{\prime}} e^{-i \vec{k} \cdot \vec{R}_{i}} e^{i \vec{k}^{\prime} \cdot \vec{R}_{j}} \frac{2}{N} \cdot \frac{1}{\omega-\Omega(\vec{K}, \vec{k})} \sum_{\vec{q}} J\left(\frac{1}{2} \vec{K}-\vec{k}-\vec{q}\right) G\left(\vec{K}, \vec{k}+\vec{q} ; \vec{K}, \vec{k}^{\prime} ; \omega\right)$

$=\frac{2}{N^{2}} \sum_{\vec{k} \vec{k}^{\prime}} e^{-i \vec{k} \cdot \vec{R}_{i}} e^{i \vec{k}^{\prime} \cdot \vec{R}_{j}} \frac{1}{\omega-\Omega(\vec{K}, \vec{k})} \sum_{\vec{q}} J\left(\frac{1}{2} \vec{K}-\vec{k}-\vec{q}\right)$

$\cdot\left[\frac{1}{N} \sum_{l m} e^{i\left(\vec{k}+\vec{q}^{\prime}\right) \cdot \vec{R}_{l}} e^{-i \vec{k}^{\prime} \cdot \vec{R}_{m}} G(l, m, \vec{K}, \omega)\right]$ 


$$
\begin{aligned}
& =\frac{2}{N} \sum_{\vec{k}, l} e^{i \frac{1}{2} \vec{K} \cdot \vec{R}_{l}-i \vec{k} \cdot \vec{R}_{i}} \frac{1}{\omega-\Omega(\vec{K}, \vec{k})}\left[\frac{1}{N} \sum_{\vec{q}} e^{-i\left(\frac{1}{2} \vec{K}-\vec{k}-\vec{q}\right) \cdot \vec{R}_{l}} J\left(\frac{1}{2} \vec{K}-\vec{k}-\vec{q}\right)\right] G(l, j, \vec{K}, \omega) \\
& =\frac{2}{N} \sum_{\vec{k}, l} J(l) \frac{e^{i \frac{1}{2} \vec{k} \cdot \vec{R}_{l}-i \vec{k} \cdot \vec{R}_{i}}}{\omega-\Omega(\vec{K}, \vec{k})} G(l, j, \vec{K}, \omega) \\
& =2 \sum_{l} J(l)\left[\frac{1}{N} \sum_{\vec{k}} \frac{\cos \frac{1}{2} \vec{K} \cdot \vec{R}_{l} \cos \vec{k} \cdot \vec{R}_{i}}{\omega-\Omega(\vec{K}, \vec{k})}\right] G(l, j, \vec{K}, \omega)
\end{aligned}
$$

So the eq. (2.17) reduces to the following Dyson's equation.

$$
\begin{aligned}
G(i, j, \vec{K}, \omega)= & 8 S^{2}\left[1-\frac{\delta_{j 0}}{2 S}\right] \Lambda(i j, \vec{K}, \omega)+2 \sum_{l} I_{l} \tilde{\Lambda}(i l, \vec{K}, \omega) G(l, j, \vec{K}, \omega) \\
& 2 \sum_{l}\left(J_{l}-I_{l}\right)[\Lambda(i l, \vec{K}, \omega)+\tilde{\Lambda}(i l, \vec{K}, \omega)] G(l, j, \vec{K}, \omega) \\
& -2 \sum_{l} D \Lambda(i l, \vec{K}, \omega) G(l, j, \vec{K}, \omega) \delta_{l 0}
\end{aligned}
$$

Where,

$$
\begin{aligned}
\Lambda(i j, \vec{K}, \omega) & =\frac{1}{N} \sum_{\vec{k}} \frac{\cos \vec{k} \cdot \vec{R}_{i} \cos \vec{k} \cdot \vec{R}_{j}}{\omega-\Omega(\vec{K}, \vec{k})} \\
\tilde{\Lambda}(i j, \vec{K}, \omega) & =\frac{1}{N} \sum_{\vec{k}} \frac{\cos \vec{k} \cdot \vec{R}_{i}\left(\cos \frac{1}{2} \vec{K} \cdot \vec{R}_{j}-\cos \vec{k} \cdot \vec{R}_{j}\right)}{\omega-\Omega(\vec{K}, \vec{k})}
\end{aligned}
$$

Equation (2.19) is the same as the eq.7 appeared in [22], and can also be expressed in terms of lattice Green's Function which is defined by,

$$
\begin{array}{r}
L_{i}(\vec{K}, \omega)=\frac{1}{N} \sum_{\vec{k}} \frac{e^{i \vec{k} \cdot \vec{R}_{i}}}{\omega-\Omega(\vec{K}, \vec{k})} \\
G(i, j, \vec{K}, \omega)=4 S^{2}\left[L_{i-j}+L_{i+j}-\frac{1}{S} L_{i} \delta_{j 0}\right] \\
-2 \sum_{l} I(l) L_{l-i} G(l, j,, \vec{K}, \omega)
\end{array}
$$




$$
\begin{aligned}
& +2 \sum_{l} J(l) \cos \left(\frac{1}{2} \vec{K} \cdot \vec{R}_{l}\right) L_{i} G(l, j, \vec{K}, \omega) \\
& -2 D L_{i} G(0, j, \vec{K}, \omega)
\end{aligned}
$$

Here we use a symmetric relation $G(l, j, \vec{K}, \omega)=G(-l, j, \vec{K}, \omega)$, which will be proved in the next chapter.

The numerical evaluation of lattice Green's functions is considered in section 3.3 after specifying the application of the Green's function formalism to the case of the triangular lattice in section 3.1 . 


\section{Chapter 3}

\section{Application to the Triangular Lattice}

\subsection{Two-Magnon Propagators with NN Interac- tion}

The Green's function $G(i, i, \vec{K}, \omega)$ represents the propagator of two-magnon process involving the creation of two spin deviations on two sites separated by a distance $\vec{R}_{i}$ (on a two dimensional lattice it is specified by two indices $\vec{R}_{i}=\left[i_{x}, i_{y}\right]$ ). The spectral function of such a process is proportional to $-\operatorname{Im} G(i, i, \vec{K}, \omega)$. In a twodimensional triangular lattice (Figure 3.1 ) we should specify the lattice site by

$$
\vec{R}_{i}=l \vec{a}+m \vec{b}
$$

Where $l+m$ is an even integer, and $a$ and $b$ are equal to $\frac{1}{2}$ and $\frac{1}{2} \sqrt{3}$ of the edge of the basic cell of triangular lattice.

In this thesis we will examine the spectra of single-ion 2 -spin deviations $\left(\vec{R}_{0}=\right.$ $[0,0])$ and also the NN deviations along the three different directions, which are 


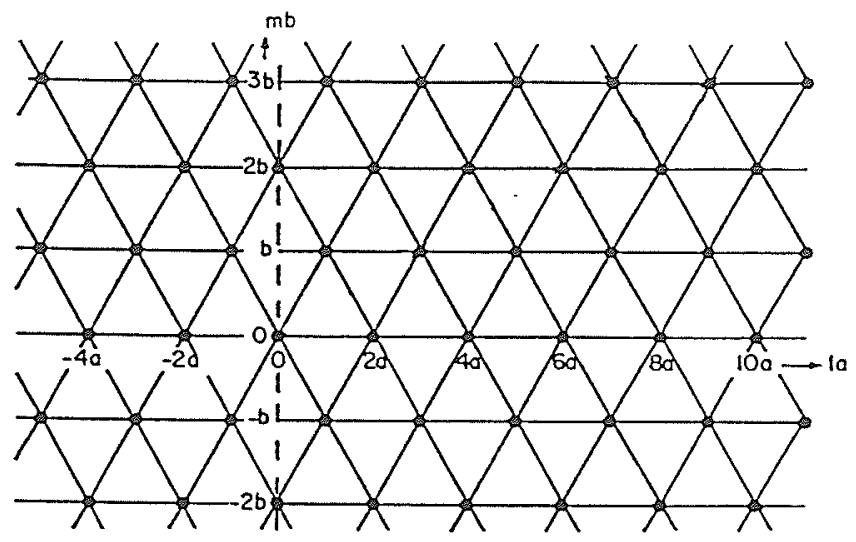

Figure 3.1: Network of the triangular lattice

$i=(2,0), i=(1,1)$, and $i=(-1,1)$. Shorthand notations for these sites, $i=0,1^{\prime}, 1^{\prime \prime}$, and $1^{\prime \prime \prime}$ are sometimes used respectively.

We would first like to explore some symmetry properties of the Green's functions, the lattice Green's functions and two-magnon energy spectral functions.

1. If $\Omega(\vec{K}, \vec{k})$ is the total energy of a system consisting of two independent magnons $\left(\vec{k}_{1}, \vec{k}_{2}\right)$, the $\vec{k}$-inversion symmetry holds for any value of $\vec{K}$, i.e. $\Omega(\vec{K}, \vec{k})=$ $\Omega(\vec{K},-\vec{k})$.

2. The Green functions have such symmetry that,

$$
G(i, j, \vec{K}, \omega)=G(i,-j, \vec{K}, \omega)=G(-i, j, \vec{K}, \omega)=G(-i,-j, \vec{K}, \omega)
$$

proof:

It follows from the definition of the 2-magnon Green's function that

$$
G\left(\vec{k}_{1}, \vec{k}_{2} ; \vec{k}_{1}^{\prime}, \vec{k}_{2}^{\prime}\right)=G\left(\vec{k}_{2}, \vec{k}_{1} ; \vec{k}_{1}^{\prime}, \vec{k}_{2}^{\prime}\right)=G\left(\vec{k}_{1}, \vec{k}_{2} ; \vec{k}_{2}^{\prime}, \vec{k}_{1}^{\prime}\right)=G\left(\vec{k}_{2}, \vec{k}_{1} ; \vec{k}_{2}^{\prime}, \vec{k}_{1}^{\prime}\right)
$$


i.e.

$G\left(\vec{K}, \vec{k} ; \vec{K}^{\prime}, \vec{k}^{\prime}\right)=G\left(\vec{K},-\vec{k} ; \vec{K}^{\prime}, \vec{k}^{\prime}\right)=G\left(\vec{K}, \vec{k} ; \vec{K}^{\prime},-\vec{k}^{\prime}\right)=G\left(\vec{K},-\vec{k} ; \vec{K}^{\prime},-\vec{k}^{\prime}\right)$

Remember that the real space Green's function is just a partial Fourier transform of the reciprocal one(eq. (2.14)), the above symmetry of $G(i, j, \vec{K}, \omega)$ is obvious.

3. Lattice Green's functions have the inversion symmetry too, i.e. $L_{m, n}(\vec{K}, \omega)=$ $L_{\bar{m}, \bar{n}}(\vec{K}, \omega)$. This is a direct extension of the inversion symmetry of the energy dispersion function.

4. Energy spectra, Green's function and lattice Green's function will become more symmetric when the total wavevector $\vec{K}=0$.

Considering only nearest neighbour interactions, that is, $I(l)=0, J(l)=0$, if $\left|\vec{R}_{l}\right|$ is greater than the first neighbour separation, and taking into account of the symmetry properties mentioned above we will see that eq. $(2.20)$ connects $G([00,[00], \vec{K}, \omega)$ with the other three off-diagonal Green's functions $G([20],[00], \vec{K}, \omega), G([11],[00], \vec{K}, \omega)$, $G([\overline{1} 1],[00], \vec{K}, \omega)$. The derivation is shown in detail as following.

Replacing the $i, j$ in eq. $(2.20)$ by $[00],[00]$,

$$
\begin{aligned}
G([00],[00], \vec{K}, \omega) \\
=\quad 4 S^{2}\left[2-\frac{1}{S}\right] L_{00} \\
\quad-2 I_{1}\left\{2 L_{20} G([20],[00], \vec{K}, \omega)+2 L_{11} G([11],[00], \vec{K}, \omega)+2 L_{\overline{1} 1} G([\overline{1} 1],[00], \vec{K}, \omega)\right\} \\
\quad+2 J_{1} L_{00}\left\{2 \cos \left(\frac{1}{2} \vec{K} \cdot \vec{R}_{20}\right) G([20],[00], \vec{K}, \omega)\right.
\end{aligned}
$$




$$
\begin{aligned}
& \left.\quad+2 \cos \left(\frac{1}{2} \vec{K} \cdot \vec{R}_{11}\right) G([11],[00], \vec{K}, \omega)+2 \cos \left(\frac{1}{2} \vec{K} \cdot \vec{R}_{\overline{1} 1}\right) G([\overline{1} 1],[00], \vec{K}, \omega)\right\} \\
& -2 D L_{00} G([00],[00], \vec{K}, \omega)
\end{aligned}
$$

Taking $\alpha=\cos \frac{1}{2} K_{x} a, \quad \beta=\cos \frac{1}{2} K_{y} b$ and letting

$$
A=2 \alpha^{2}-1, \quad B=\alpha \cdot \beta, \quad C=\sqrt{\left(1-\alpha^{2}\right)\left(1-\beta^{2}\right)}
$$

Then,

$$
\begin{gathered}
\cos \frac{1}{2} \vec{K} \cdot \vec{R}_{20}=\cos K_{x} a=A \\
\cos \frac{1}{2} \vec{K} \cdot \vec{R}_{11}=\cos \left(\frac{1}{2} K_{x} a+\frac{1}{2} K_{y} b\right)=B-C \\
\cos \frac{1}{2} \vec{K} \cdot \vec{R}_{-11}=\cos \left(-\frac{1}{2} K_{x} a+\frac{1}{2} K_{y} b\right)=B+C
\end{gathered}
$$

We have,

$$
\begin{aligned}
(1+ & \left.2 D L_{00}\right) \cdot G([00],[00], \vec{K}, \omega)+4\left(I_{1} L_{20}-J_{1} L_{00} \cdot A\right) \cdot G([20],[00], \vec{K}, \omega) \\
& +4\left(I_{1} L_{11}-J_{1} L_{00} \cdot(B-C)\right) \cdot G([11],[00], \vec{K}, \omega) \\
& +4\left(I_{1} L_{\overline{1} 1}-J_{1} L_{00} \cdot(B+C)\right) \cdot G([\overline{1} 1],[00], \vec{K}, \omega)=4 S^{2}\left(2-\frac{1}{S}\right) L_{00}
\end{aligned}
$$

The same way we get the equation for $G([20],[00], \vec{K}, \omega)$

$$
\begin{aligned}
& G([20],[00], \vec{K}, \omega) \\
&=4 S^{2}\left[2-\frac{1}{S}\right] L_{20} \\
&-2 I_{1}\left\{\left(L_{00}+L_{40}\right) G([20],[00], \vec{K}, \omega)+\left(L_{\overline{1} 1}+L_{31}\right) G([11],[00], \vec{K}, \omega)\right. \\
&\left.+\left(L_{11}+L_{3 \overline{1}}\right) G([\overline{1} 1],[00], \vec{K}, \omega)\right\} \\
&+2 J_{1} L_{20}\left\{2 \cos \left(\frac{1}{2} \vec{K} \cdot \vec{R}_{20}\right) G([20],[00], \vec{K}, \omega)\right.
\end{aligned}
$$




$$
\begin{aligned}
& \left.\quad+2 \cos \left(\frac{1}{2} \vec{K} \cdot \vec{R}_{11}\right) G([11],[00], \vec{K}, \omega)+2 \cos \left(\frac{1}{2} \vec{K} \cdot \vec{R}_{\overline{1}_{1}}\right) G([\overline{1} 1],[00], \vec{K}, \omega)\right\} \\
& -2 D L_{20} G([00],[00], \vec{K}, \omega)
\end{aligned}
$$

It leads to

$$
\begin{aligned}
2 D & L_{20} \cdot G([00],[00], \vec{K}, \omega) \\
+ & \left\{1+2 I_{1}\left(L_{00}+L_{40}\right)-4 J_{1} L_{20} \cdot A\right\} \cdot G([20],[00], \vec{K}, \omega) \\
+ & \left\{2 I_{1}\left(L_{1 \overline{1}}+L_{31}\right)-4 J_{1} L_{20} \cdot(B-C)\right\} \cdot G([11],[00], \vec{K}, \omega) \\
+ & \left\{2 I_{1}\left(L_{11}+L_{3 \overline{1}}\right)-4 J_{1} L_{20} \cdot(B+C)\right\} \cdot G([\overline{1} 1],[00], \vec{K}, \omega)=4 S^{2}\left(2-\frac{1}{S}\right) L_{20}
\end{aligned}
$$

Replacing $i, j$ in eq. $(2.20)$ by [11], [00],

$$
\begin{aligned}
G([11],[00], \vec{K}, \omega) \\
=\quad 4 S^{2}\left[2-\frac{1}{S}\right] L_{11} \\
-2 I_{1}\left\{\left(L_{1 \overline{1}}+L_{31}\right) G([20],[00], \vec{K}, \omega)+\left(L_{00}+L_{22}\right) G([11],[00], \vec{K}, \omega)\right. \\
\left.+\left(L_{20}+L_{02}\right) G([\overline{1} 1],[00], \vec{K}, \omega)\right\} \\
+2 J_{1} L_{11}\left\{2 \cos \left(\frac{1}{2} \vec{K} \cdot \vec{R}_{20}\right) G([20],[00], \vec{K}, \omega)\right. \\
\left.+2 \cos \left(\frac{1}{2} \vec{K} \cdot \vec{R}_{11}\right) G([11],[00], \vec{K}, \omega)+2 \cos \left(\frac{1}{2} \vec{K} \cdot \vec{R}_{\overline{11}}\right) G([\overline{1} 1],[00], \vec{K}, \omega)\right\} \\
-2 D L_{11} G([00],[00], \vec{K}, \omega)
\end{aligned}
$$

It leads to

$$
2 D L_{11} \cdot G([00],[00], \vec{K}, \omega)+\left\{2 I_{1}\left(L_{1 \overline{1}}+L_{31}\right)-4 J_{1} L_{11} \cdot A\right\} \cdot G([20],[00], \vec{K}, \omega)
$$




$$
\begin{aligned}
& +\left\{1+2 I_{1}\left(L_{00}+L_{22}\right)-4 J_{1} L_{11} \cdot(B-C)\right\} \cdot G([11],[00], \vec{K}, \omega) \\
& +\left\{2 I_{1}\left(L_{20}+L_{02}\right)-4 J_{1} L_{11} \cdot(B+C)\right\} \cdot G([11],[00], \vec{K}, \omega)=4 S^{2}\left(2-\frac{1}{S}\right) L_{11}
\end{aligned}
$$

The last we have

$$
\begin{aligned}
G([1 \overline{1}], & {[00], \vec{K}, \omega) } \\
= & 4 S^{2}\left[2-\frac{1}{S}\right] L_{\overline{1} 1} \\
& -2 I_{1}\left\{\left(L_{11}+L_{3 \overline{1}}\right) G([20],[00], \vec{K}, \omega)+\left(L_{20}+L_{02}\right) G([11],[00], \vec{K}, \omega)\right. \\
& \left.+\left(L_{00}+L_{2 \overline{2}}\right) G([\overline{1} 1],[00], \vec{K}, \omega)\right\} \\
+ & 2 J_{1} L_{1 \overline{1}}\left\{2 \cos \left(\frac{1}{2} \vec{K} \cdot \vec{R}_{20}\right) G([20],[00], \vec{K}, \omega)\right. \\
& \left.+2 \cos \left(\frac{1}{2} \vec{K} \cdot \vec{R}_{11}\right) G([11],[00], \vec{K}, \omega)+2 \cos \left(\frac{1}{2} \vec{K} \cdot \vec{R}_{\overline{1} 1}\right) G([\overline{1} 1],[00], \vec{K}, \omega)\right\} \\
- & 2 D L_{1 \overline{1}} G([00],[00], \vec{K}, \omega)
\end{aligned}
$$

It leads to

$$
\begin{aligned}
2 D & L_{1 \overline{1}} \cdot G([00],[00], \vec{K}, \omega)+\left\{2 I_{1}\left(L_{11}+L_{3 \overline{1}}\right)-4 J_{1} L_{1 \overline{1}} \cdot A\right\} \cdot G([20],[00], \vec{K}, \omega) \\
& +\left\{2 I_{1}\left(L_{20}+L_{02}\right)-4 J_{1} L_{1 \overline{1}} \cdot(B-C)\right\} \cdot G([11],[00], \vec{K}, \omega) \\
& +\left\{1+2 I_{1}\left(L_{00}+L_{2 \overline{2}}\right)-4 J_{1} L_{1 \overline{1}} \cdot(B+C)\right\} \cdot G([\overline{1} 1],[00], \vec{K}, \omega)=4 S^{2}\left(2-\frac{1}{S}\right) L_{\overline{1} 1}
\end{aligned}
$$

Define the factor functions:

$$
\begin{aligned}
F_{0}\left(L_{a}, L_{b}, v\right) & =4 I_{1}\left(L_{a}-\sigma \cdot L_{b} \cdot v\right) \\
F_{1}\left(L_{a}, L_{b}, L_{c}, v\right) & =2 I_{1}\left(L_{a}+L_{b}-2 \sigma \cdot L_{c} \cdot v\right)
\end{aligned}
$$


where $\sigma$ is the Ising parameter, $L_{a}, L_{b}, L_{c}$ specify different lattice Green functions and $v$ is a variable which would be replaced by $A, B-C$ or $B+C$.

Combining from eq. (3.2) to eq. (3.5) we have the following matrix equation:

$$
\underline{(I+Q) \cdot G=R}
$$

where $I$ is the identity matrix and,

$$
G=\left(\begin{array}{c}
G([00],[00], \vec{K}, \omega) \\
G([20],[00], \vec{K}, \omega) \\
G([11],[00], \vec{K}, \omega) \\
G([11],[00], \vec{K}, \omega)
\end{array}\right)
$$

The coefficient matrix $Q$ equals

$$
\left(\begin{array}{rrrr}
2 D \cdot L_{00} & F_{0}\left(L_{20}, L_{00}, A\right) & F_{0}\left(L_{11}, L_{00}, B-C\right) & F_{0}\left(L_{\overline{1} 1}, L_{00}, B+C\right) \\
2 D \cdot L_{20} & F_{1}\left(L_{00}, L_{40}, L_{20}, A\right) & F_{1}\left(L_{1 \overline{1}}, L_{31}, L_{20}, B-C\right) & F_{1}\left(L_{11}, L_{3 \overline{1}}, L_{20}, B+C\right) \\
2 D \cdot L_{11} & F_{1}\left(L_{1 \overline{1}}, L_{31}, L_{11}, A\right) & F_{1}\left(L_{00}, L_{22}, L_{11}, B-C\right) & F_{1}\left(L_{20}, L_{02}, L_{11}, B+C\right) \\
2 D \cdot L_{1 \overline{1}} & F_{1}\left(L_{11}, L_{3 \overline{1}}, L_{1 \overline{1}}, A\right) & F_{1}\left(L_{20}, L_{02}, L_{1 \overline{1}}, B-C\right) & F_{1}\left(L_{00}, L_{\overline{2} 2}, L_{1 \overline{1}}, B+C\right)
\end{array}\right)
$$

and the inhomogeneous term,

$$
R=4 S^{2}\left(2-\frac{1}{S}\right)\left(\begin{array}{l}
L_{00} \\
L_{20} \\
L_{11} \\
L_{1 \overline{1}}
\end{array}\right)
$$

We may notice that, taking any combination of the indices $\mathbf{i}$ and $\mathbf{j}$, equation (2.20) always connects $G(i, j, \vec{K}, \omega)$ with a set of Green's functions $G(l, j, \vec{K}, \omega)(l=$ all NN sites) and $G(0, j, \vec{K}, \omega)$ through the matrix equation. Only the first term of r.h.s. of eq. (2.20) contributes to the inhomogeneous column matrix $R$. The other four terms give us the coefficient matrix $\mathbf{Q}$. Furthermore, only the first index $\mathbf{i}$ affects the matrix $\mathrm{Q}$ by factoring different lattice Green's functions in the front of $G(l, j, \vec{K}, \omega)$ and 
$G(0, j, \vec{K}, \omega)$. while the second index $\mathbf{j}$ only goes into the column matrix $\mathbf{R}$. In other words if we keep the same arrangement of the related Green's functions, such as,

$$
G=\left(\begin{array}{l}
G([00], j, \vec{K}, \omega) \\
G([20], j, \vec{K}, \omega) \\
G([11], j, \vec{K}, \omega) \\
G([\overline{1} 1], j, \vec{K}, \omega)
\end{array}\right)
$$

(it is a typical type of arrangement in the problem of determining the single-ion and NN deviation propagators in the 2-d triangular lattice) we will have exactly the same coefficient matrix $Q$, no matter what $\mathbf{j}$ is involved. Therefore eq. (3.6) is actually a universal equation in determining all four Green functions we desire. We may rewrite it as,

$$
(I+Q) \cdot G=R_{j}
$$

Let $j=[2,0]$,

$$
R_{20}=4 S^{2}\left(\begin{array}{l}
2 L_{20} \\
L_{00}+L_{40} \\
L_{1 \overline{1}}+L_{31} \\
L_{11}+L_{3 \overline{1}}
\end{array}\right) \quad \text { and } \quad G=\left(\begin{array}{l}
G([00],[20], \vec{K}, \omega) \\
G([20],[20], \vec{K}, \omega) \\
G([11],[20], \vec{K}, \omega) \\
G([\overline{1} 1],[20], \vec{K}, \omega)
\end{array}\right)
$$

The second term of the column matrix above is the propagator of NN excitation along the direction $\vec{R}=[2,0]$.

Let $j=[1,1]$,

$$
R_{11}=4 S^{2}\left(\begin{array}{l}
2 L_{11} \\
L_{1 \overline{1}}+L_{31} \\
L_{00}+L_{22} \\
L_{02}+L_{20}
\end{array}\right) \quad \text { and } \quad G=\left(\begin{array}{l}
G([00],[11], \vec{K}, \omega) \\
G([20],[11], \vec{K}, \omega) \\
G([11],[11], \vec{K}, \omega) \\
G([\overline{1} 1],[11], \vec{K}, \omega)
\end{array}\right)
$$

The third term of the column matrix above is the propagator of NN excitation along the direction $\vec{R}=[1,1]$. 
Let $j=[\overline{1}, 1]$,

$$
R_{\overline{1} 1}=4 S^{2}\left(\begin{array}{l}
2 L_{1 \overline{1}} \\
L_{11}+L_{3 \overline{1}} \\
L_{02}+L_{20} \\
L_{00}+L_{2 \overline{2}}
\end{array}\right) \quad \text { and } \quad G=\left(\begin{array}{l}
G([00],[\overline{1} 1], \vec{K}, \omega) \\
G([20],[\overline{1} 1], \vec{K}, \omega) \\
G([11],[\overline{1} 1], \vec{K}, \omega) \\
G([\overline{1} 1],[\overline{1} 1], \vec{K}, \omega)
\end{array}\right)
$$

The fourth term of the column matrix above is the propagator of NN excitation along the direction $\vec{R}=[\overline{1}, 1]$.

\subsection{Critical Points in the Two-magnon Spectra}

The critical points are important in the structure of two-magnon propagators and their evolution as a function of $\vec{K}$. We define the density of states or spectral function of any excitation by

$$
\rho(\omega)=\frac{1}{N} \sum_{\vec{k}} \delta\left(\omega-\omega_{\vec{k}}\right)
$$

Here, $\vec{k}$ is wave vector, and $\omega_{\vec{k}}$ is the dispersion relation.

Replace the summation by the integration over the 1-st BZ,

$$
\rho(\omega)=\frac{V}{(2 \pi)^{3}} \int_{\omega} \frac{d s}{\left|\vec{\nabla} \omega_{\vec{k}}\right|}
$$

The critical point is defined by $\left|\vec{\nabla} \omega_{\vec{k}}\right|=0$, which leads to the singularities in the density of states. They were first studied by van Hove[30] in the context of oneparticle excitations.

Differentiating equation (3.14) with respect to $a x$ and by we obtain the following equations for the critical points:

$$
\frac{\partial \omega}{\partial a x} \propto 2 A \sin a x \cos a x+B \sin a x \cos b y-C \cos a x \sin b y=0
$$




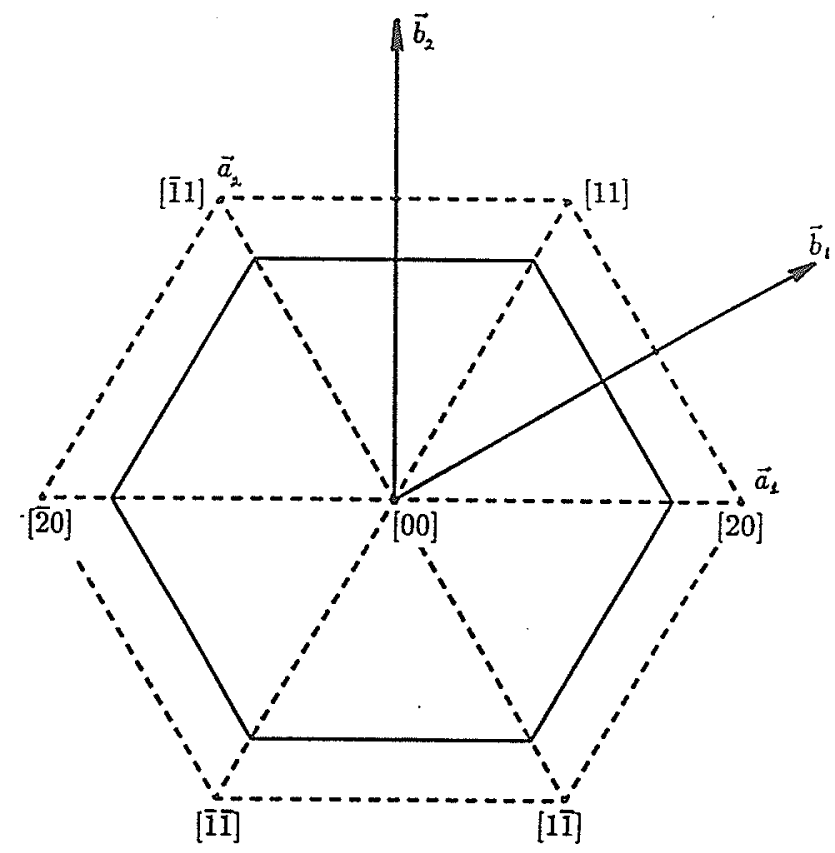

The dashed lines represent the real lattice, the solid lines represent the first Brillouin zone, while $\vec{b}_{1} \vec{b}_{1}$ are the reciprocal vectors of $\vec{a}_{1} \vec{a}_{2}$

Figure 3.2: The basic cell of the real space lattice and 1-st the brillouin Zone

$$
\frac{\partial \omega}{\partial b y} \propto B \cos a x \sin b y-C \sin a x \cos b y=0
$$

They must be satisfied simultaneously.

Combining the two equations to eliminate $\cos a x \sin b y$,

$$
2 A B \sin a x \cos a x-\left(C^{2}-B^{2}\right) \sin a x \cos b y=0
$$

With some simple algebra, we obtain the following solutions of critical points which can be divided into two groups. The one is given by $\sin a x=\sin b y=0$, or $\cos a x=\cos b y=0 \quad$ for all values of $\alpha$ and $\beta$. They correspond to the highsymmetry points $\Gamma$ (the center of 1 -st $\mathrm{BZ}$ ) and $M, M^{\prime}, M^{\prime \prime}$ in $\vec{k}$ space (Figure 3.2), 
These have energies,

$$
\begin{gathered}
\Gamma(a x=0, b y=0): \quad \omega_{\Gamma}=8 S I_{1}\left\{3-\sigma_{1}\left(2 \alpha^{2}-1\right)-2 \sigma_{1} \alpha \beta\right\}+2(2 S-1) D \\
M(a x=0, b y=\pi): \quad \omega_{\mathrm{M}}=8 S I_{1}\left\{3-\sigma_{1}\left(2 \alpha^{2}-1\right)+2 \sigma_{1} \alpha \beta\right\}+2(2 S-1) D \\
M^{\prime}(\pi / 2, \pi / 2): \quad \omega_{\mathrm{M}^{\prime}}=8 S I_{1}\left\{3+\sigma_{1}\left(2 \alpha^{2}-1\right)-2 \sigma_{1} \sqrt{\left(1-\alpha^{2}\right)\left(1-\beta^{2}\right)}\right\}+2(2 S-1) D \\
M^{\prime \prime}(-\pi / 2, \pi / 2): \quad \omega_{\mathrm{M}^{\prime \prime}}=8 S I_{1}\left\{3+\sigma_{1}\left(2 \alpha^{2}-1\right)+2 \sigma_{1} \sqrt{\left(1-\alpha^{2}\right)\left(1-\beta^{2}\right)}\right\}+2(2 S-1) D
\end{gathered}
$$

In contrast to these four solutions the remaining critical points move in $\vec{k}$ space as $\alpha, \beta$ varies. They are determined by the set of equations:

$$
\left\{\begin{array}{l}
2 A B \cos a x=\left(C^{2}-B^{2}\right) \cos b y \\
2 A C \sin a x=\left(C^{2}-B^{2}\right) \sin b y
\end{array}\right.
$$

We need to study some different cases.

(a) $B \neq 0, C \neq 0$, and $B \neq C$,

Substituting the first equation into the second one we have,

$$
\left(C^{2}-B^{2}\right)^{2}\left(1-\frac{4 A^{2} B^{2}}{\left(C^{2}-B^{2}\right)^{2}} \cos ^{2} a x\right)=(2 A C)^{2} \sin ^{2} a x
$$

Then,

$$
\cos ^{2} a x\left[4 A^{2} B^{2}-4 A^{2} C^{2}\right]=\left(C^{2}-B^{2}\right)^{2}-4 A^{2} C^{2}
$$

So,

$$
\begin{aligned}
\cos ^{2} a x & =\frac{B^{2}-C^{2}}{4 A^{2}}-\frac{C^{2}}{B^{2}-C^{2}} \\
& =\frac{\alpha^{2}+\beta^{2}-1}{4\left(2 \alpha^{2}-1\right)^{2}}-\frac{\left(1-\alpha^{2}\right)\left(1-\beta^{2}\right)}{\alpha^{2}+\beta^{2}-1}
\end{aligned}
$$


Let

$$
(a x)^{*}=\arccos \left\{\frac{B^{2}-C^{2}}{4 A^{2}}-\frac{C^{2}}{B^{2}-C^{2}}\right\}^{1 / 2}
$$

We then define $(b y)^{\star}$ with,

$$
(b y)^{*}=\arccos \left(\left|\frac{2 A B}{C^{2}-B^{2}} \cos a x\right|\right)
$$

Here $0<(a x)^{\times}<\pi / 2, \quad 0<(b y)^{\times}<\pi / 2$. Now we need to choose the appropriate quadrants of $a x$, and by to satisfy the original equation set which can be rewriten as:

$$
\left\{\begin{array}{l}
\cos b y=\frac{2 A B}{C^{2}-B^{2}} \cos a x \\
\sin b y=\frac{2 A C}{C^{2}-B^{2}} \sin a x
\end{array}\right.
$$

When $C<B$

- $\cos b y$ has a different sign from $\cos a x$.

- $\sin b y$ has a different sign from $\sin a x$.

So we have four possible sets of solutions. They are

$$
\begin{array}{ll}
\left(a x=a x^{*}\right. & \left., b y=\pi+b y^{*}\right) \\
\left(a x=\pi-a x^{*}\right. & \left., b y=-b y^{*}\right) \\
\left(a x=\pi+a x^{*}\right. & \left., b y=b y^{*}\right) \\
\left(a x=-a x^{*}\right. & \left., b y=\pi-b y^{*}\right)
\end{array}
$$

It can be shown that they are equivalent points (they can be transformed to one point by inversing or moving by some units of reciprocal lattice vectors).

when $C>B$

- $\cos b y$ has same sign with $\cos a x$. 
- $\sin b y$ has same sign with $\sin a x$.

So the four possible solutions are:

$$
\begin{array}{ll}
\left(a x=a x^{\star},\right. & \left.b y=b y^{\star}\right) \\
\left(a x=\pi-a x^{*},\right. & \left.b y=\pi-b y^{\star}\right) \\
\left(a x=\pi+a x^{*},\right. & \left.b y=\pi+b y^{\star}\right) \\
\left(a x=-a x^{*},\right. & \left.b y=-b y^{\star}\right)
\end{array}
$$

Again they are equivalent as well.

(b) $B=0, C \neq 0$

In this case we have:

$$
\left\{\begin{array}{l}
\cos b y=0 \\
C \sin b y=2 A \sin a x
\end{array}\right.
$$

It leads to:

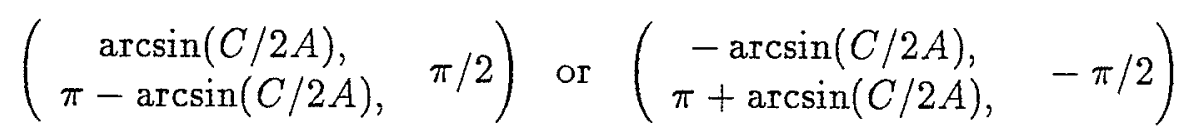

They are equivalent to each other.

(c) $B \neq 0, C=0$

We have

$$
\left\{\begin{array}{l}
\sin b y=0 \\
-B \cos b y=2 A \cos a x
\end{array}\right.
$$

And then, the solutions are:

$$
\left(\begin{array}{ll}
\pi-\arccos (B / 2 A), & 0 \\
\pi+\arccos (B / 2 A), & 0
\end{array}\right) \text { or } \quad\left(\begin{array}{rr}
-\arccos (B / 2 A), & \pi \\
\arccos (B / 2 A), & \pi
\end{array}\right)
$$

They are also equivalent.

(d) $B=C=0(\alpha=1, \beta=0)$ 
In this case the original formulae for $\partial \omega / \partial a x$, and $\partial \omega / \partial b y$ reduce to

$$
\sin 2 a x=0 \Longrightarrow a x=0, \pi / 2
$$

The corresponding energies:

$$
\omega=\left\{\begin{array}{lll}
8 S I_{1}\left(3-\sigma_{1}\right) & \text { for } & a x=0 \\
8 S I_{1}\left(3+\sigma_{1}\right) & \text { for } & a x=\pi / 2
\end{array}\right.
$$

These two critical points are not in isolated type.

(e) $B=C \neq 0$

That means:

$$
\left\{\begin{array}{l}
\cos a x=0 \\
\sin a x=0
\end{array} \Longrightarrow\right. \text { NO SOLUTION }
$$

A SAS program was writen to draw the critical points along the high-symmetry directions $\Gamma \longrightarrow X \longrightarrow M \longrightarrow \Gamma$. And here $\Gamma=(\vec{K} \cdot \vec{a}=0, \vec{K} \cdot \vec{b}=0), \quad X=(\vec{K} \cdot \vec{a}=$ $\pi / 3, \vec{K} \cdot \vec{b}=\pi), \quad M=(\vec{K} \cdot \vec{a}=0, \vec{K} \cdot \vec{b}=\pi)$. Figure 3.3 shows the picture of critical lines in the absence of anisotropy.

The diagrams of critical lines in square lattice [24] and 3-D SC lattice[31] have been studied by other workers before. With the isotropic NN interaction they both consist of only straightlines which correspond to high symmetry points $\Gamma, X, M$ (and $R$ in 3-D SC), and both have zero bandwidth at the Brilliouin Zone corner. For the SC lattice these lines begin to bend and the critical lines which don't correspond to the high-symmetry points appear only after the next nearest neighbour (NNN) coupling $(\eta)$ is taken into account. But in triangular lattice the complicated nature of lattice makes both of two groups of critical lines as well as a non-zero minimum bandwidth 
exist even when only NN isotropic interactions are present. A similiar phenomenon is also found for NN FCC magnet[32].

\subsection{Lattice Green Functions}

The evaluation of the lattice Green functions turns out to be the kernel of the computation of two-magnon propagators.

The 2-magnon dispersion function (eq. 1.9) with NN interaction on the triangular lattice is given by,

$$
\begin{aligned}
\omega(\vec{K}, \vec{k})= & 8 S I_{1}\left\{3-\sigma_{1} \cos \vec{K} \cdot \vec{a} \cos 2 a x-\sigma_{1} \cos \frac{1}{2} \vec{K} \cdot(\vec{a}+\vec{b}) \cos (a x+b y)\right. \\
& \left.-\sigma_{1} \cos \frac{1}{2} \vec{K} \cdot(-\vec{a}+\vec{b}) \cos (-a x+b y)\right\}+2(2 S-1) D \\
= & 8 S I_{1}\left\{3-\sigma_{1} \cos \vec{K} \cdot \vec{a} \cos 2 a x-2 \sigma_{1} \cos \frac{1}{2} \vec{K} \cdot \vec{a} \cos \frac{1}{2} \vec{K} \cdot \vec{b} \cos a x \cos b y\right. \\
& \left.-2 \sigma_{1} \sin \frac{1}{2} \vec{K} \cdot \vec{a} \sin \frac{1}{2} \vec{K} \cdot \vec{b} \sin a x \sin b y\right\}+2(2 S-1) D
\end{aligned}
$$

Using the parameters defined in eq.3.1 the dispersion relation can be written as,

$$
\begin{aligned}
\omega(\vec{K}, \vec{k})= & 8 S I_{1}\left\{3-\sigma_{1}\left(2 \alpha^{2}-1\right) \cos 2 a x-2 \sigma_{1} \alpha \beta \cos a x \cos b y\right. \\
& \left.-2 \sigma_{1} \sqrt{\left(1-\alpha^{2}\right)\left(1-\beta^{2}\right)} \sin a x \sin b y+2(2 S-1) D\right\} \\
= & 8 S I_{1}\left\{3-\sigma_{1} A \cos 2 a x-2 \sigma_{1} B \cos a x \cos b y-2 \sigma_{1} C \sin a x \sin b y\right\}+2(2 S-1) D
\end{aligned}
$$

So the lattice Green function,

$$
\begin{aligned}
& L_{l m}(\omega+i \varepsilon)=\frac{a b}{(2 \pi)^{2}} \int_{-\pi / a}^{\pi / a} d x \int_{-\pi / a}^{\pi / a} d y \\
& \quad \frac{\cos (l a x+m b y)}{\omega-8 S I_{1}\left\{3-\sigma_{1} A \cos 2 a x-2 \sigma_{1} B \cos a x \cos b y-2 \sigma_{1} C \sin a x \sin b y\right\}-2(2 S-1) D}
\end{aligned}
$$



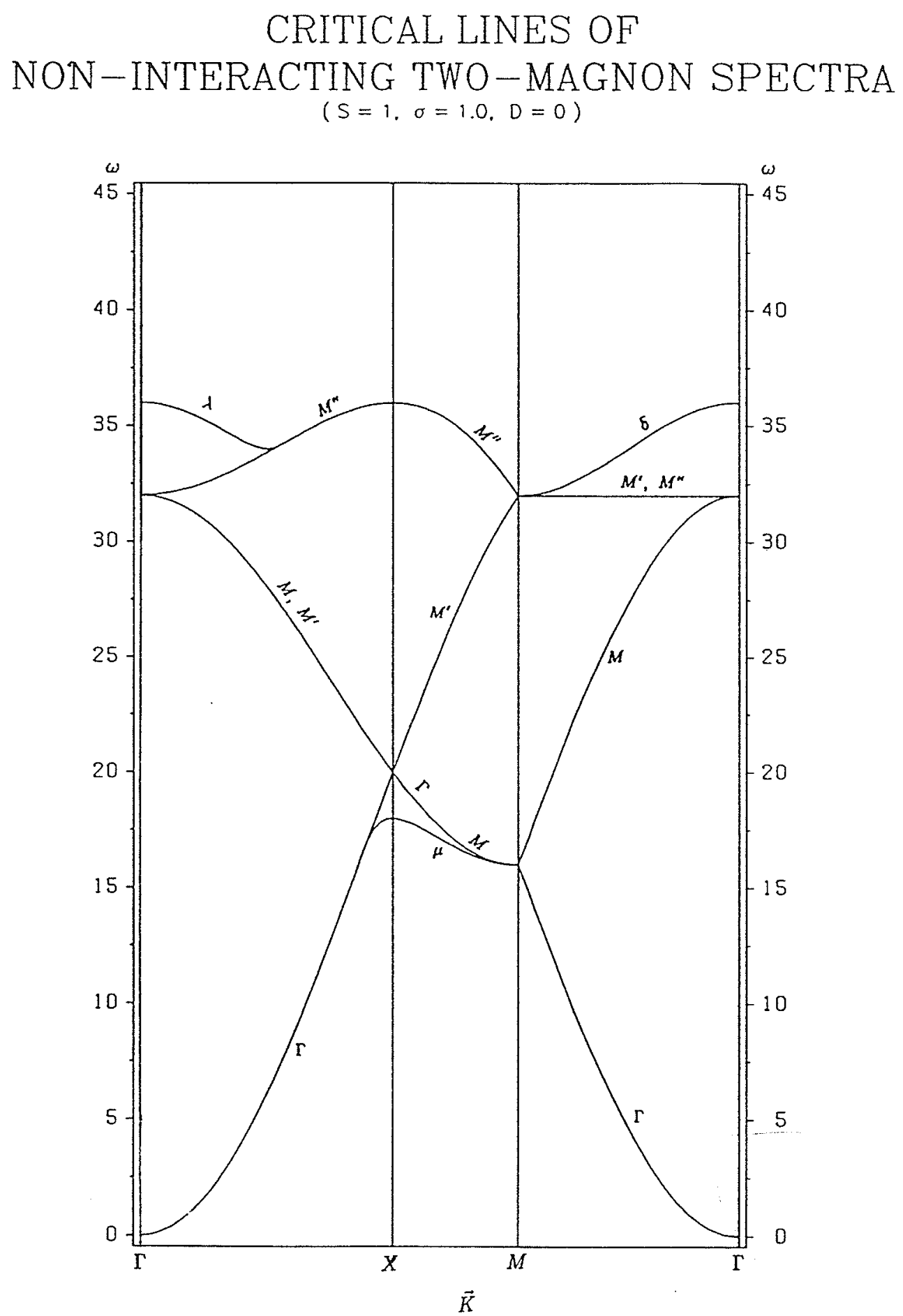

Figure 3.4: Critical Points 
We set up a standard form of lattice Green function by,

$$
L_{l m}(\omega+i \varepsilon)=-\frac{1}{8 S I_{1} \sigma_{1}} H(l, m ; \omega-i \varepsilon)
$$

Where,

$$
\begin{gathered}
H(l, m ; \omega)=\frac{1}{(2 \pi)^{2}} \int_{-\pi}^{\pi} d x \int_{-\pi}^{\pi} d y \cdot \frac{\cos (l x+m y)}{t-\omega_{H}(\vec{K}, \vec{k})} \\
\omega_{H}(\vec{K}, \vec{k})=A \cos 2 x-2 B \cos x \cos y-2 C \sin x \sin y \\
t=\frac{1}{8 S I_{1} \sigma_{1}}\left\{24 S I_{1}+2(2 S-1) D-\omega\right\}
\end{gathered}
$$

Some numerical schemes were developed to evaluate the Brillouin Zone integrals. However, analytical expressions are available in some special cases. Such as when $\vec{K}=0$

$$
H(l, m ; \omega)=\frac{1}{\pi^{2}} \int_{0}^{\pi} d x \int_{0}^{\pi} d y \cdot \frac{\cos l x \cos m y}{t-\cos 2 x-2 \cos x \cos y}
$$

Which can be expressed in terms of the complete elliptic integrals of the first, second, and third kind. The formulae to determine the first three lattice Green functions $H(0,0), H(2,0), H(4,0)$ and a recurrence relation to connect other high-index ones are given by Horiguchi[33]. Readers who are interested in the method for evaluation of the complete elliptic integrals with a complex modulus can refer to Horiguchi[34] and Bulirsch's work[35]. A Fortran program is also attached in the appendix of this thesis.

At $\mathrm{M}$ point(Fig. 3.2), where $K_{x}=0, K_{y}=\pi$,

$$
\alpha=1, \quad \beta=0
$$


So,

$$
\begin{gathered}
\omega(\vec{K}, \vec{k})=8 S I_{1}\left(3-\sigma_{1} \cos 2 x\right) \\
H_{i j}(t)=\frac{1}{(2 \pi)^{2}} \int_{-p i}^{\pi} d x \int_{-p i}^{\pi} d y \frac{\cos (i x+j y)}{t-\cos 2 x}
\end{gathered}
$$

Apparently, when $j \neq 0, \quad H_{i j}=0$. When both $i=0$ and $j=0($ see $[36])$

$$
\begin{aligned}
H_{00} & =\frac{1}{(2 \pi)^{2}} \int_{-p i}^{\pi} d x d y \frac{1}{t-\cos 2 x} \\
& =\frac{1}{\pi} \int_{0}^{\pi} \frac{d x}{t-\cos 2 x} \\
& =\left\{\begin{aligned}
\frac{1}{\sqrt{t^{2}-1}} & (t>1) \\
\frac{i}{\sqrt{1-t^{2}}} & (-1<t<1) \\
-\frac{1}{\sqrt{t^{2}-1}} & (t<-1)
\end{aligned}\right.
\end{aligned}
$$

Other LGF's can be expressed in term of $L_{00}$, such as,

$$
\begin{gathered}
H_{20}=\frac{1}{(2 \pi)^{2}} \int_{-\pi}^{\pi} d x d y \frac{\cos 2 x}{t-\cos 2 x} \\
=\frac{1}{(2 \pi)^{2}} \int_{-\pi}^{\pi} d x d y\left[\frac{t}{t-\cos 2 x}-1\right] \\
=t \cdot L_{00}-1 \\
H_{40}=\frac{1}{(2 \pi)^{2}} \int_{-\pi}^{\pi} d x d y \frac{\cos 4 x}{t-\cos 2 x} \\
=\frac{1}{(2 \pi)^{2}} \int_{-\pi}^{\pi} d x d y \frac{2 \cos 2 x-1}{t-\cos 2 x} \\
=\frac{1}{(2 \pi)^{2}} \int_{-\pi}^{\pi} d x d y \frac{2\left(\cos ^{2} 2 x-t^{2}\right)+2 t^{2}-1}{t-\cos 2 x} \\
=\left(2 t^{2}-1\right) \cdot L_{00}-\frac{1}{(2 \pi)^{2}} \int_{-\pi}^{\pi} d x d y(\cos 2 x+t) \\
=\left(2 t^{2}-1\right) \cdot L_{00}-2 t
\end{gathered}
$$


High usage of CPU time is required to compute lattice Green functions for general $\vec{K}$ 's. Note that the general form of two-dimensional spectral functions is

$$
\begin{aligned}
G(E) & =\frac{1}{N} \sum_{\vec{k}} \frac{F(\vec{k})}{E-\omega(\vec{k})+i \varepsilon} \\
& =\frac{1}{N} \mathbf{P}\left(\sum_{\vec{k}} \frac{F(\vec{k})}{E-\omega(\vec{k})}\right)-i \pi\left(\frac{1}{N} \sum_{\vec{k}} F(\vec{k}) \delta(E-\omega(\vec{k}))\right)
\end{aligned}
$$

Where $\mathbf{P}$ stands for the principal value.

We may introduce the generating function $Z(E)$ as

$$
Z(E)=\frac{1}{N} \sum_{\vec{k}} F(\vec{k}) \theta(E-E(\vec{k}))
$$

where the $\theta$-function is the Heaviside step function (eq. 2.5). The imaginary part of the spectral function $\operatorname{Im} G(E)$ is then immediately obtained from the energy derivative of $Z(E)$,

$$
\operatorname{Im} G(E)=-\pi \frac{d Z(E)}{d E}
$$

So the problem is changed to the computation of a typical surface integral $Z(E)$, to compute the total area of 1-st Brillouin Zone where $E>E(\vec{k})$. In this thesis this is done with the triangular linear analytic method[37] in which the irreducible part of the 1-st Brillouin Zone is divided into small triangular cells and the contributions from each cell are summed. The program can be verified by taking $\vec{K}=0$ (Figure 3.5) and comparing the results with Horiguchi's method(Figure. 3.4). $\sigma=1$, and $D=0$ are chosen in both of two figures.

Some interesting recurrence relations of the lattice Green functions for a general 
REAL \& IMAGINARY PART OF LATTICE GREEN S FUNCTION

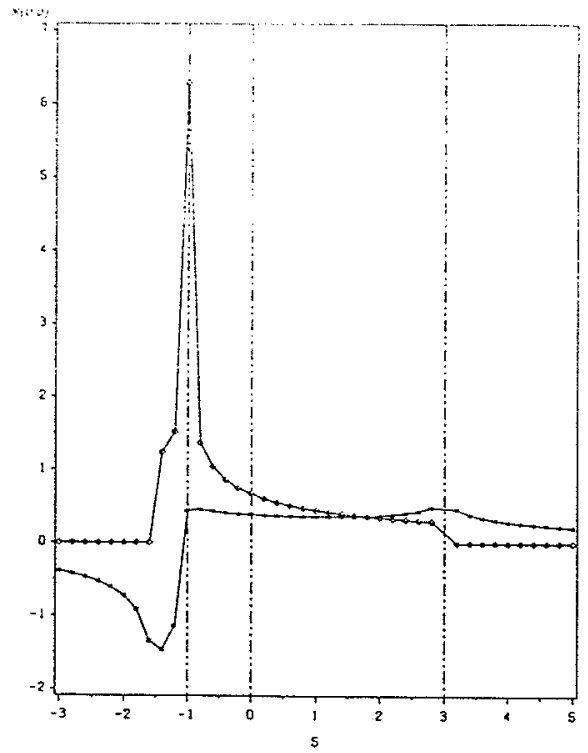

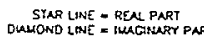

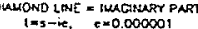

REAL \& MAGINARY PART OF LATTICE GREEN S FUNCTION

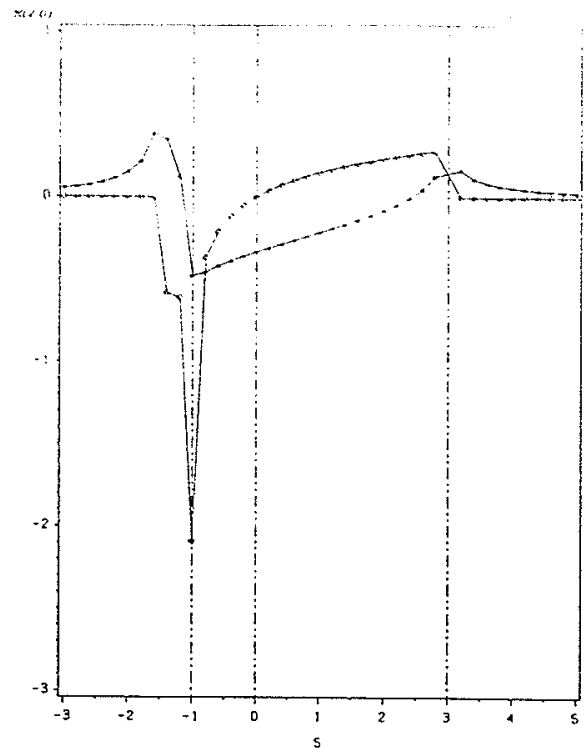

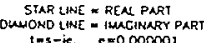

ins -ie. $\quad=0.000001$

REAL \& MAGINARY PART OF LATTICE GREEN S FUNCTION

xis:

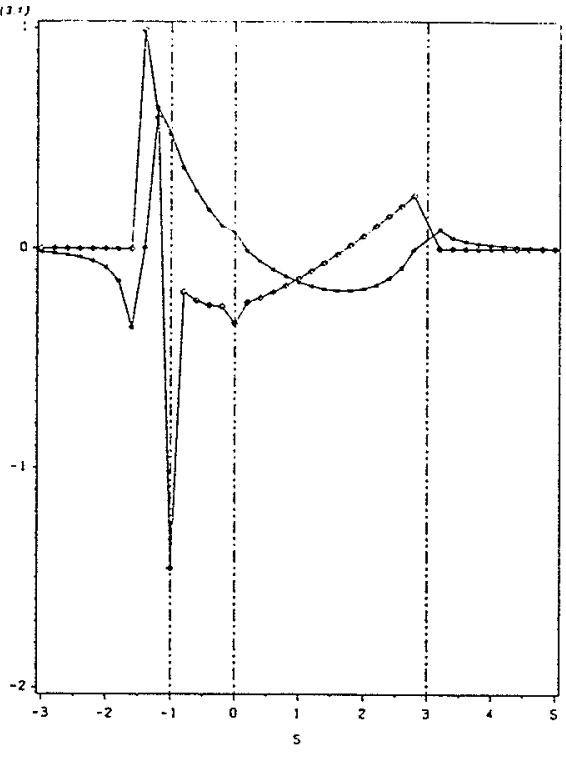

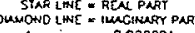

-is. $e=0.000001$

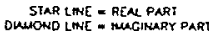

REAL \& IMAGINARY PART OF LATTICE GREEN S FUNCTION

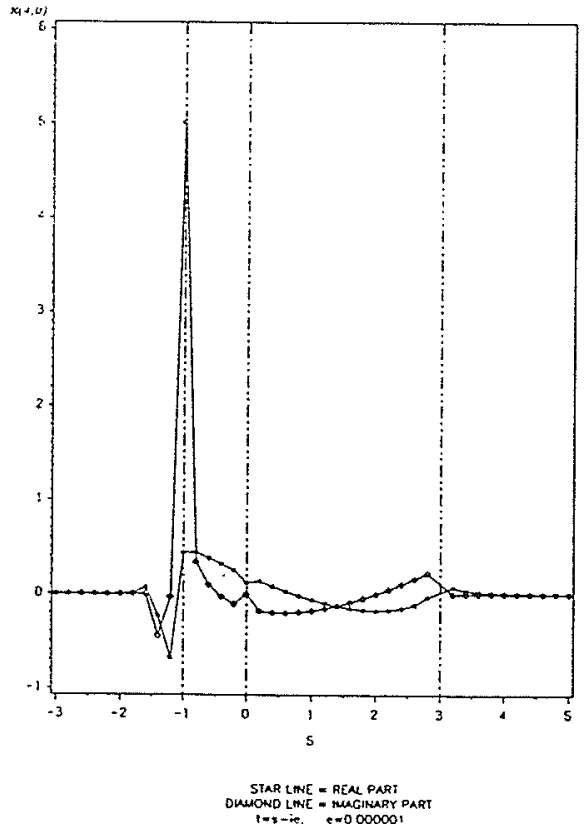

Figure 3.4: Lattice Green's Functions Calculated in Terms of Elliptic Integrals with Horiguchi's Method 
REAL \& IMAGINARY PART OF LATTICE GREEN S FUNCTION

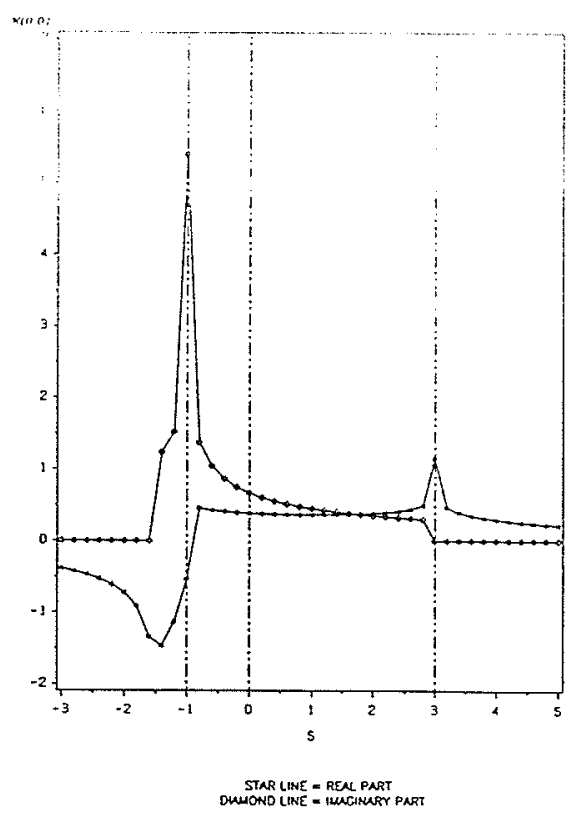

REAL \& IMAGINARY PART OF LATTICE GREEN S FUNCTION

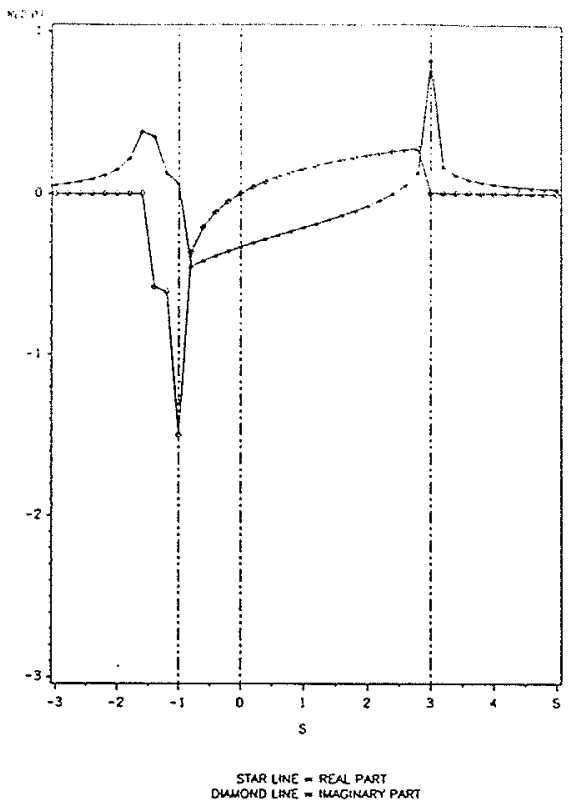

REAL \& MAGINARY PART OF LATTICE GREEN S FUNCTION REAL \& MAGINARY PART OF LATTICE GREEN S FUNCTION
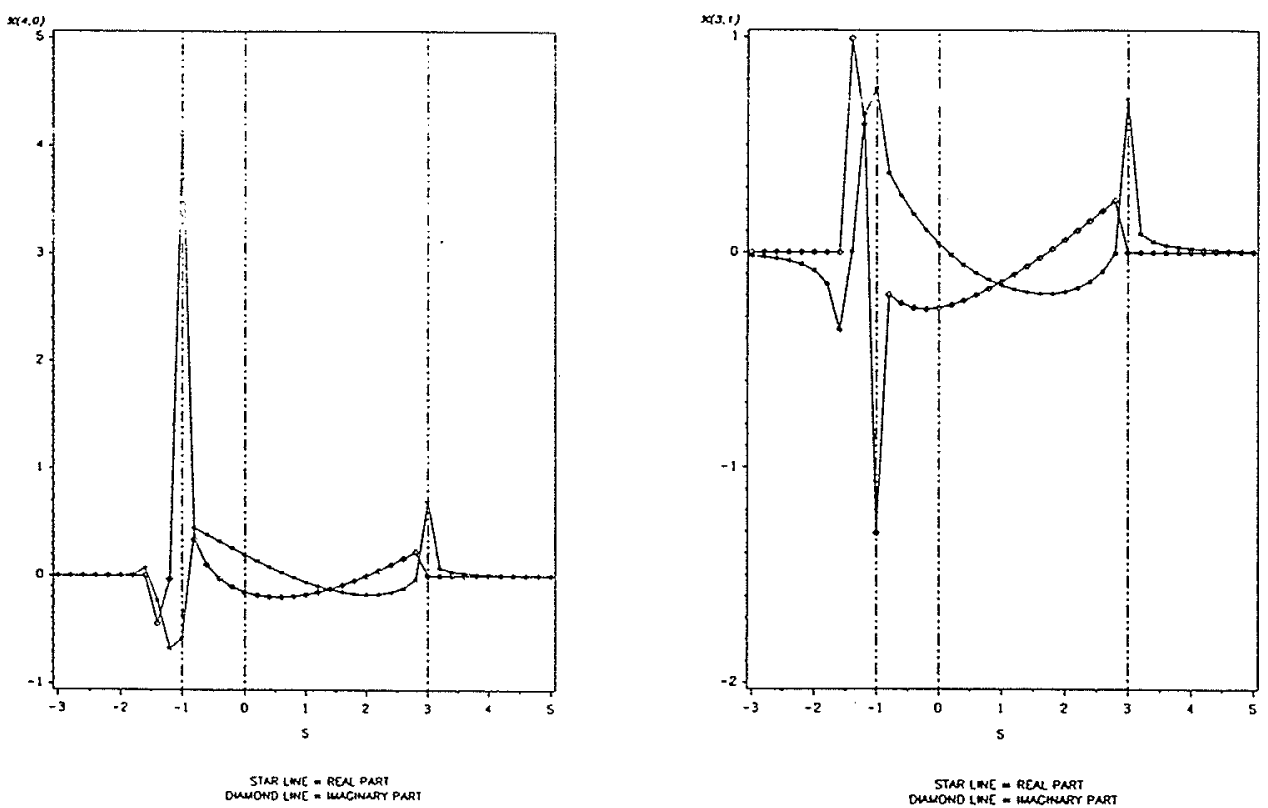

Figure 3.5: Lattice Green's Functions Calculated Numerically using the Triangular Linear Analytic Method 
$\vec{K} \neq 0$ can be developed. Note the identity,

$$
0=\sum_{\vec{k}} \frac{t-\omega_{H}(\vec{K}, \vec{k})}{t-\omega_{H}(\vec{K}, \vec{k})} \cdot \cos (l x+m y)=t H(l, m)-\sum_{\vec{k}} \frac{\omega_{H}(\vec{K}, \vec{k}) \cos (l x+m y)}{t-\omega_{H}(\vec{K}, \vec{k})}
$$

Substituting equation (3.17) for $\omega_{H}$, it is straightforward to show that,

$$
\begin{aligned}
2 t H(l, m)= & A\{L(l+2, m)+L(l-2, m)\} \\
& +(B-C)\{L(l+1, m+1)+L(l-1, m-1)\} \\
& +(B+C)\{L(l-1, m+1)+L(l+1, m-1)\}
\end{aligned}
$$

It provides another way to check the results of numerical calculation. 


\section{Chapter 4}

\section{Results and Discussion}

The main computational effort is associated with the evaluation and storage of the 10 LGF's for different $\vec{K}$ and $\omega$ values. Then equations (3.6) to (3.9) are solved so $G(0,0), G(1,1), G\left(1^{\prime}, 1^{\prime}\right)$ are obtained. The LGF's can be solved analytically for some special points such as: $\vec{K}=0$ and $\vec{K}=(0, \pi)$ (labeled as $M$, the middle point of the side of 1-st BZ. see Figure 3.2). So we can get the data on these points more easily and more precisely.

As $\vec{K}$ changes from $\Gamma \longrightarrow X \longrightarrow M \longrightarrow \Gamma$, the width of the continuum changes, and the resonances evolve. Bound states are found in certain regions.

\subsection{Two-Magnon Spectrum in the Absence of Anisotropy}

Fig. 4.1 shows the continuum spectrum of $G(0,0), G(1,1)$ in the isotropic case where $D=0, \sigma=1$ for $\vec{K}=0$ and $S=1$. With the unit of energy $(\omega)$ chosen as $I_{1}$, the peak of $G(0,0)$ is found at $\omega=27.8$. And the peak of $G(1,1)$ is at $\omega=30.8$. 
The critical point $(\omega=32.0)$ is associated with a sharp drop in both $G(0,0)$ and $G(1,1)$ and creates a shoulder above this point. Fig. 4.2 shows a case close to the Ising limit where we find the energy levels of $G(0,0), G(1,1)$ at $\omega=24$ and $\omega=22$ respectively. Note that from Figure $4.1(\sigma=1)$ to 4.2 (near-Ising) the peaks of $G(0,0)$ and $G(1,1)$ reverse their order, reflecting the fact that the single-ion two-magnon excitation becomes more and more bound than the NN excitation as Ising-anisotropy becomes stronger( $\sigma$ decreases $)$.

Figure 4.3 shows how the resonance peaks evolve as the function of $\vec{K}$ in the absence of anisotropy. When $\vec{K} \neq 0$ the excitations along different directions are certainly non-isotropic so that the degeneracy at $K=0$ is lifted by the appearance of a second NN excitation spectra $G\left(1^{\prime}, 1^{\prime}\right)$. All the of single-ion and nearest-neighbour resonances move down as $|\vec{K}|$ increases. The critical lines have a very strong influence on evolution of the spectrum. In Figure $4.3, G(1,1)$ and $G\left(1^{\prime}, 1^{\prime}\right)$ resonances approach the critical line $M \& M^{\prime}$ (Fig. 3.3) at around 0.40 to $0.60(\Gamma \longrightarrow X)$. While the main peak with most of the weight is restrained below the critical line, an additional hump shows up on the upper side of critical line. The same thing also happens to the $G(0,0)$ resonance when it reachs the critical line between 0.80 to $0.90(\Gamma \longrightarrow X)$.

Apparently the main resonances of neither $G(0,0)$, nor $G(1,1)$, nor $G\left(1^{\prime}, 1^{\prime}\right)$ appears to penetrate the critical line. However $G(1,1)$ shows a stronger repulsion with the critical line such that it passes down across both $G(0,0)$ and $G\left(1^{\prime}, 1^{\prime}\right)$ on the way to the zone corner. After the zone corner $(\mathrm{X})$, some weight builds up following the critical line $M^{\prime}$ (Fig. 3.3) and finally joins with the upper humps which just penetrate 


\section{TWO MAGNON PROPAGATORS IN 2-D TRIANGULAR LATTICE \\ $($ SIGMA $=J 1 / 11=1.0, S=1,0=0.0, K=0.0)$}

\section{$-\operatorname{IM}(G(0,0))$ \\ $-I M(G(1,1))$}

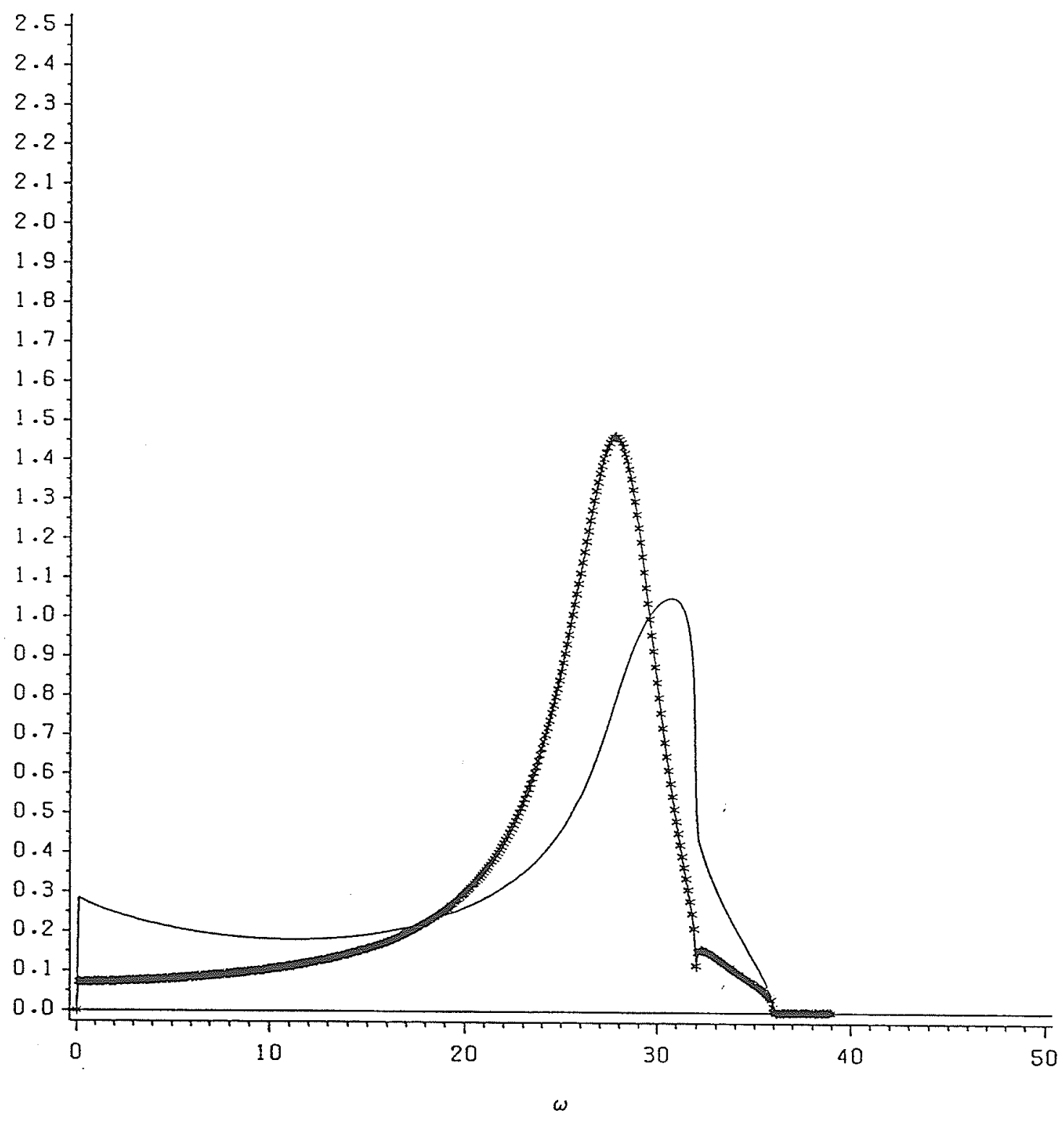

STAR LINE - - - $-\operatorname{lm}(G(0,0))$

SOLID LINE -..- $\operatorname{lm}(G(1,1))$

Figure 4.1: Two-magnon spectra at $K=0$ in the absence of anisotropy 


\section{TWO MAGNON PROPAGATORS IN 2-D TRIANGULAR LATTICE $($ SIGMA $=J 1 / 11=0.1, \quad S=1, \quad D=0.0)$}

$-\operatorname{IM}(G(0,0))$

$-I M(G(1,1))$

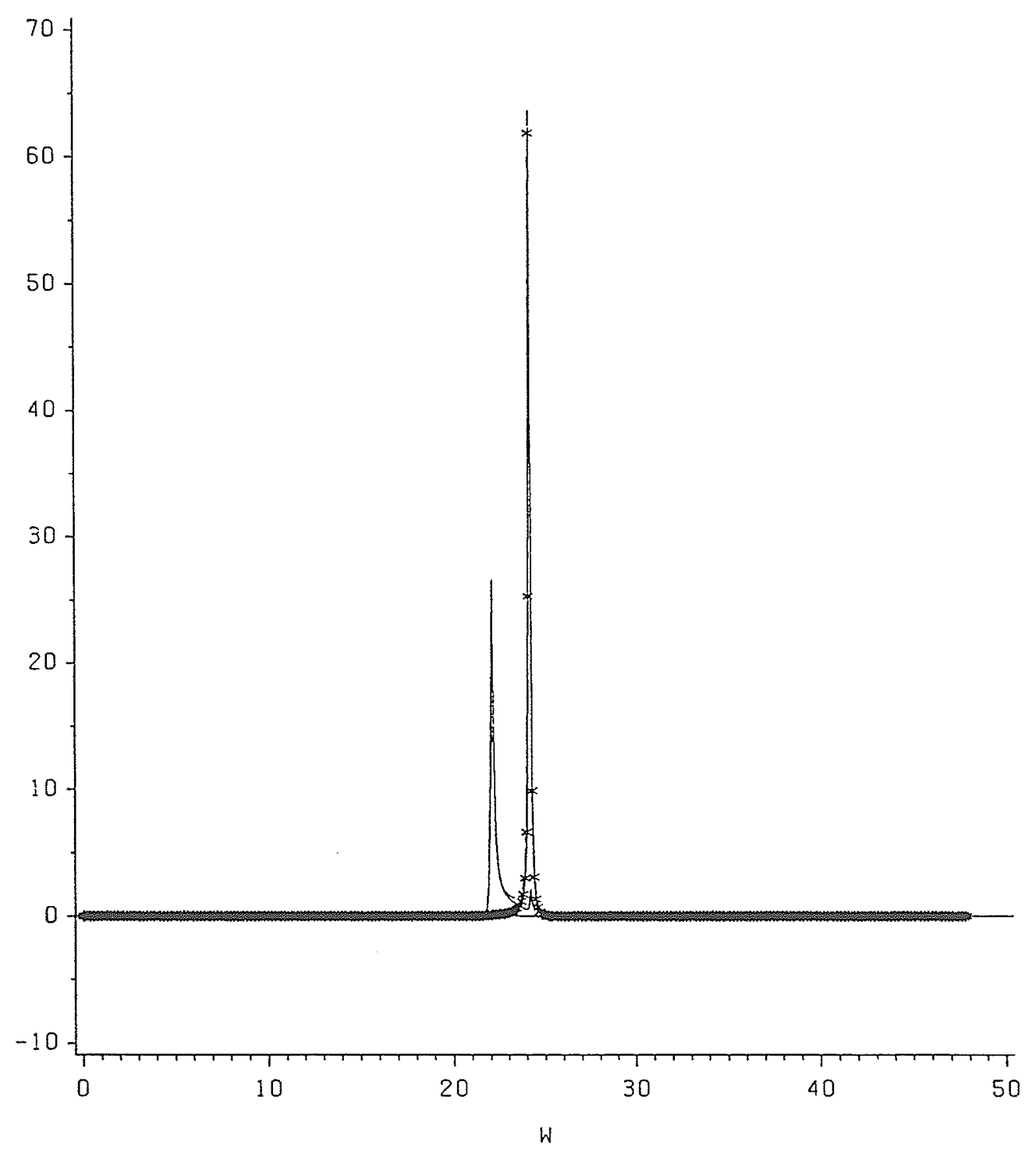

STAR LINE - - - $-\operatorname{lm}(G(0,0))$
SOLID LINE - - - $-\operatorname{lm}(G(1,1))$

Figure 4.2: Ising energy levels in the absence of anisotropy $(\sigma=0.1, D=0$ and $K=0$ ) 


\section{TWO-MAGNON DISPERSION RELATION \\ $(S=1, \sigma=1.0 .0=0)$}

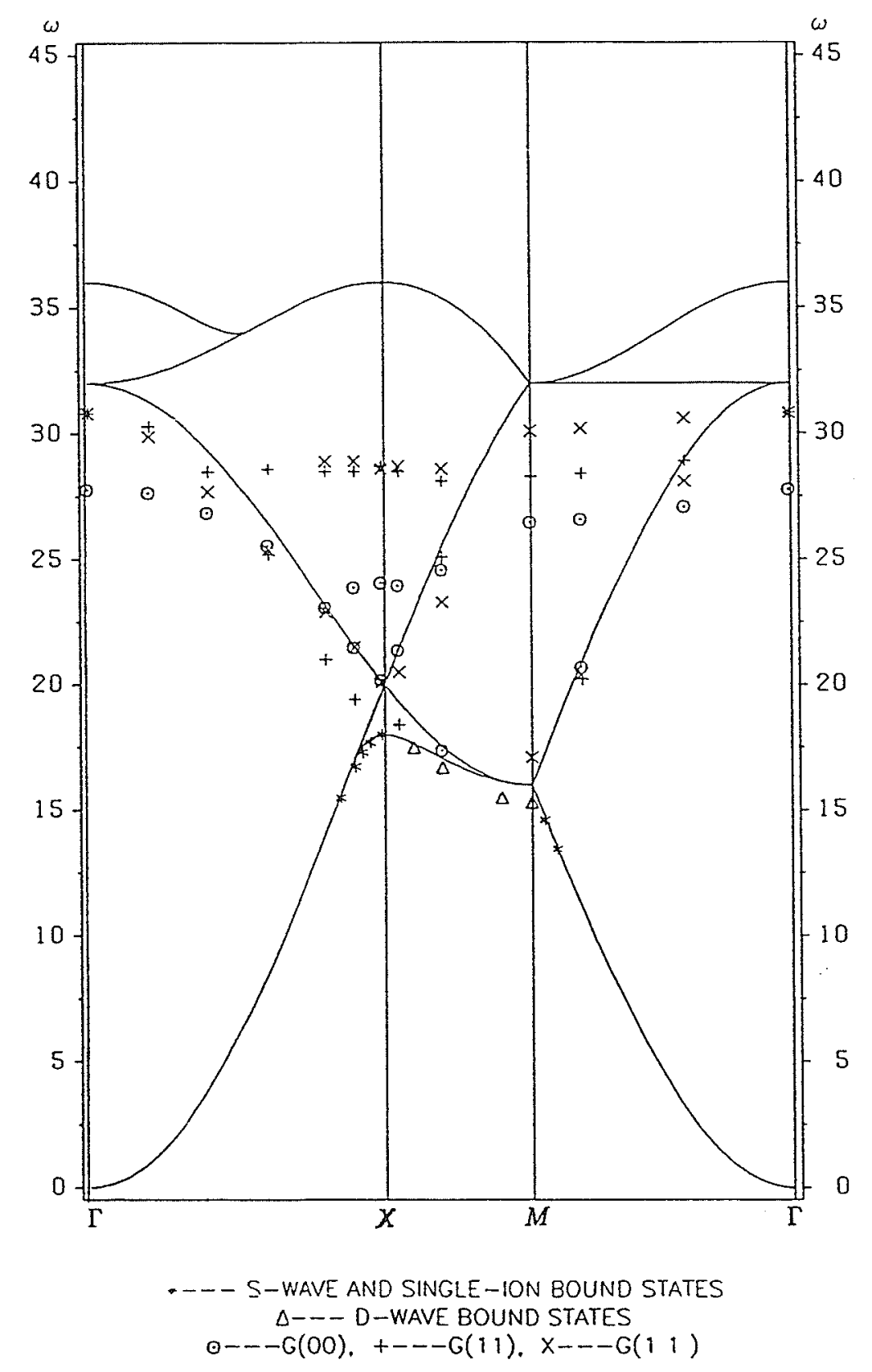

Figure 4.3: Dispersion of resonance peaks and bound states for $S=1$ in the absence of anisotropy 
TWO MAGNON PROPAGATORS IN $2-D$ TRIANGULAR LATTICE $-I H(c(0,0))$
$-I \mu(\llbracket(t, 1))$

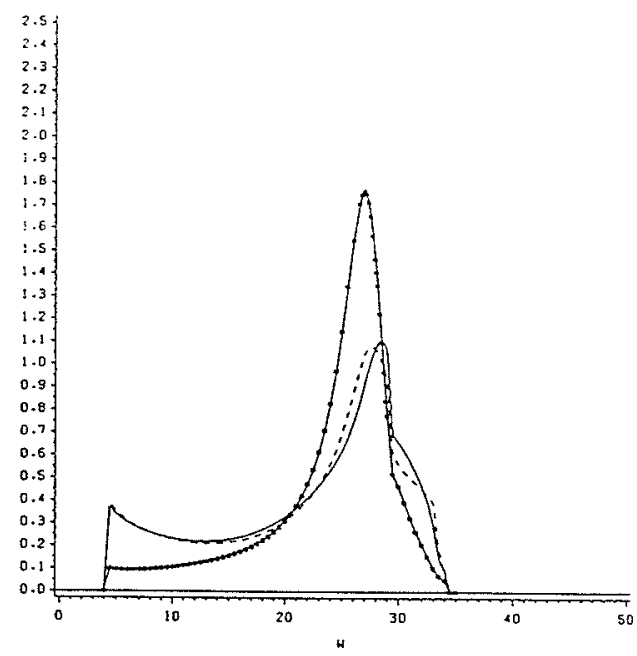

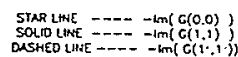

TWO MAGNON PROPAGATORS IN 2-D TRIANGULAR LATTICE

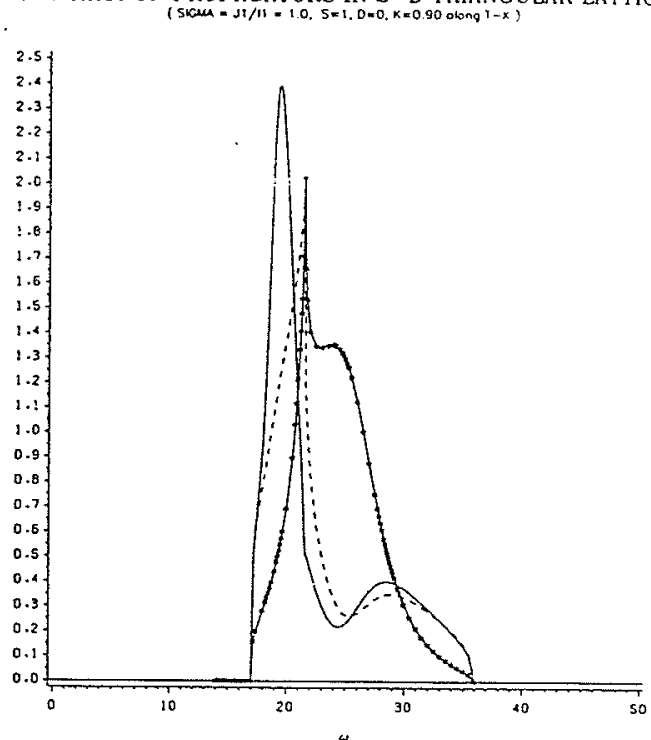

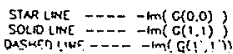

TWO MAGNON PROPAGATORS IN $2-\mathrm{D}$ TRIANGULAR LATTICE $-1+(c(0,0))$
$-I N(c(r, 1))$
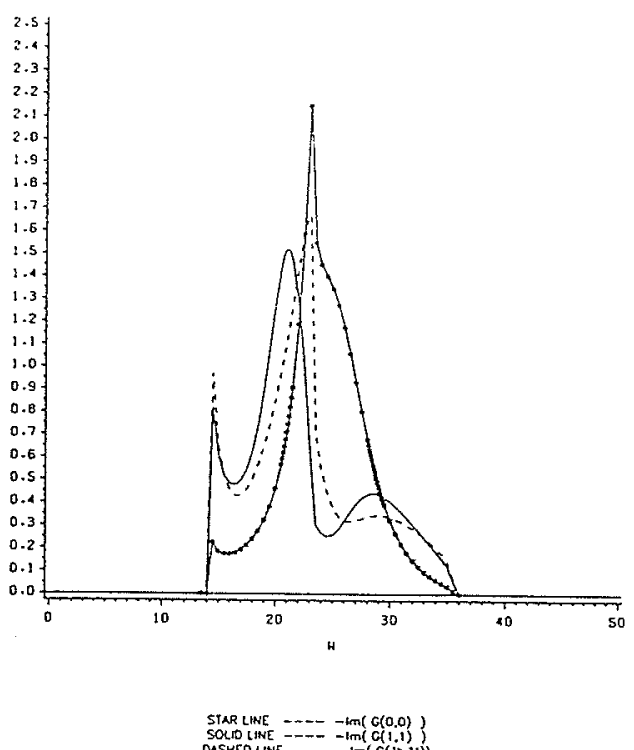

TWO MAGNON PROPAGATORS IN 2-D TRIANGULAR LATTICE

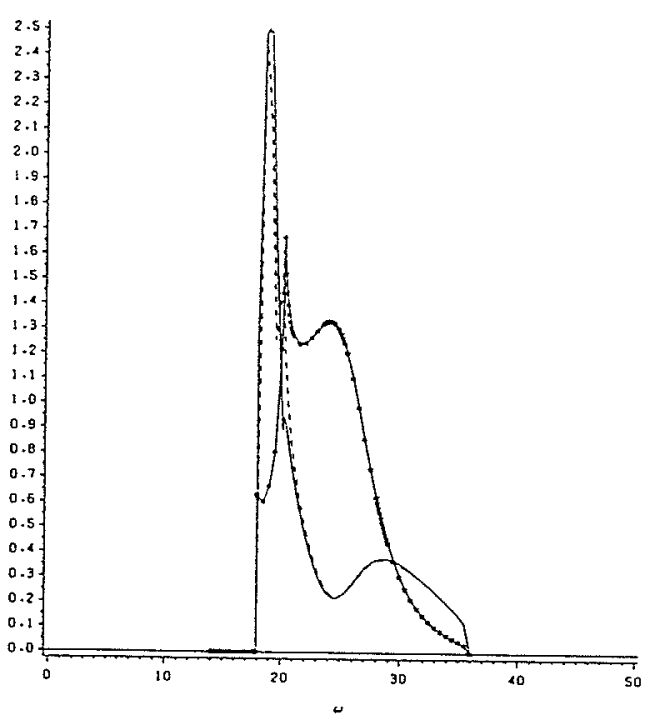

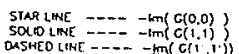

Figure 4.4: The evolution of two-magnon propagators in the absence of anisotropy 
TWO MAGNON PROPAGATORS IN 2-D TRIANGULAR LATTICE

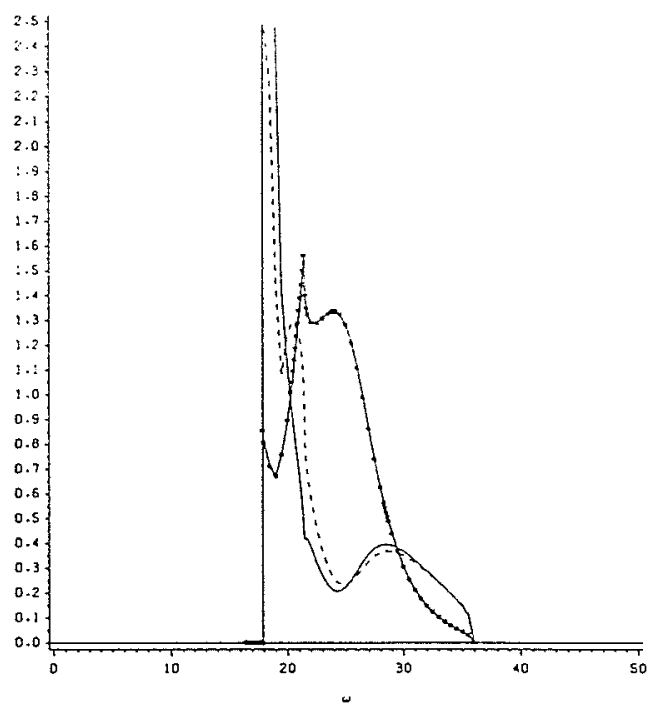

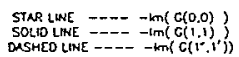

TWO MAGNON PROPAGATORS IN 2-D TRIANGULAR LATTICE
(SGM $-11 / 1=10.5=1,0 \neq 00$, v-POH: $)$

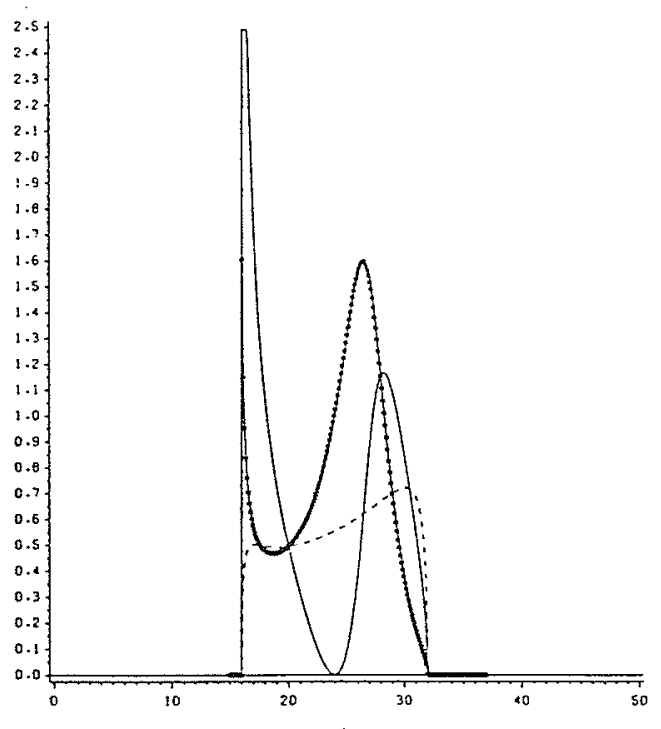

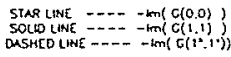

TWO MAGNON PROPAGATORS IN 2-D TRIANGULAR LATTICE

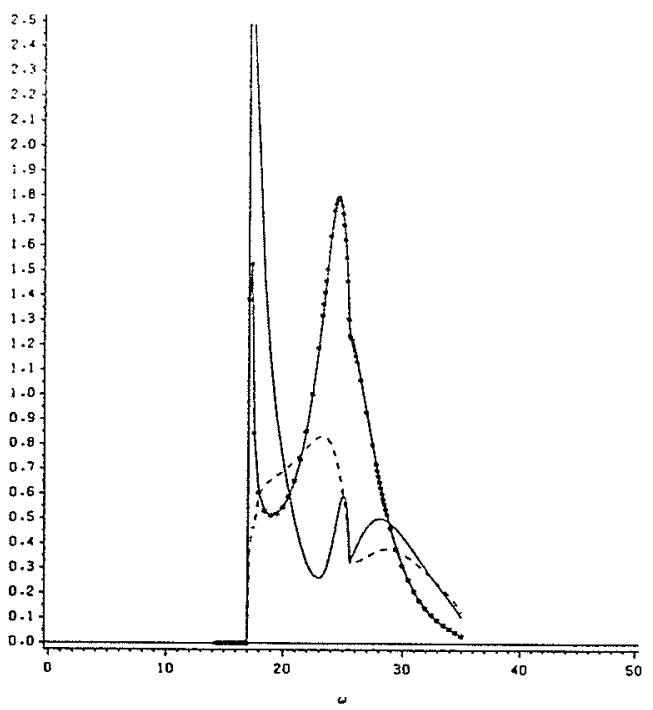

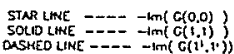

TWO MAGNON PROPAGATORS IN 2-D TRIANGULAR LATTICE

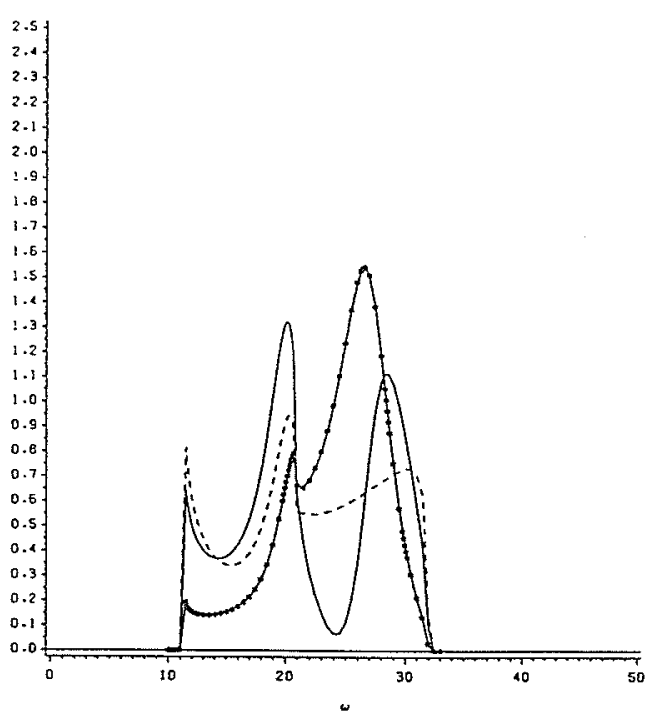

STAR LNE
SOLD LNE $-\cdots . .-\operatorname{tm}(G(O, 0))$ 
$M^{\prime}$-line. Other peaks again pick up from the bottom at $\vec{K}=(0, \pi)$ (M-point) and climb another critical line $(M)$. Eventually all peaks join together as we go back to $\Gamma$-the center of 1 -st BZ, our starting point. Figure 4.4 shows the spectrum of twomagnon propagators in the absence of anisotropy for some selected $\vec{K}$ values which forms the basis for the preceeding Figure 4.3.

Bound states are usually the focus of two-magnon calculation, largely because it is the most dramatic change when taking consideration of magnon-magnon interaction. With our Green's function method the bound state is expressed by the $\delta$-function singularity of the imaginary part of Green's function located out of the boundaries of continuum. As indicated in equation 2.10, the real part of Green's function exhibits the behaviour of $\frac{1}{x}$-type function around this point. Since the $\delta$-function spectra of $\operatorname{Im} G(i, i, \vec{K}, \boldsymbol{\omega})$ is usually too narrow to be detected, so that in practice the bound state is determined by checking the sign change of $\operatorname{Re} G(i, i, \vec{K}, \omega)$.

In our study of the 2-D triangular lattice only one bound state is found for each $\vec{K}$ in the absence of anisotropy $(\sigma=1.0, D=0.0)$. Figure 4.3 shows that the bound states begin to be detected when $\vec{K}$ moves close to $\mathrm{X}$, the corner of 1 -st $\mathrm{BZ}$. It keeps very close to the bottom of continuum, and seems join the continuum at the $\mathrm{X}$ $\left(\vec{K}=\left[\frac{1}{3} \pi, \pi\right]\right)$, then seperate further in the $X \longrightarrow M$ regions. But these bound states should be considered as two branches rather than one branch, since they are different types in different regions, they are poles of all three Green's function propagators $G(0,0), G(1,1), G\left(1^{\prime}, 1^{\prime}\right)$ in $\Gamma \longrightarrow X$ and also $M \longrightarrow \Gamma$ regions, but the poles of only the propagator $G(0,0)$ in $X \longrightarrow M$ region. We adopt the common-used 
Table 4.1: Bound states for $S=1$ in the absence of anisotropy

\begin{tabular}{|l||c|c|c|c|c|c|c|c|c|}
\hline \multicolumn{1}{|c||}{ K values } & \multicolumn{10}{|c|}{$\Gamma \longrightarrow X$} \\
\cline { 2 - 11 } & 0.0 & 0.20 & 0.40 & 0.80 & 0.85 & 0.90 & 0.925 & 0.95 & $0.99(\approx 1)$ \\
\hline Lower Boundary & 1.04 & 4.03 & 8.58 & 14.13 & 15.59 & 17.06 & 17.55 & 17.83 & 18.0 \\
\hline \hline Bound States & & & & & & & & & \\
\cline { 1 - 11 }$(0,0)$ & no & no & no & no & 15.5 & 16.72 & 17.3 & 17.7 & $*$ \\
\hline$G(1,1)$ & no & no & no & no & 15.5 & 16.72 & 17.3 & 17.7 & * \\
\hline$G\left(1^{\prime}, 1^{\prime}\right)$ & no & no & no & no & 15.5 & 16.72 & 17.3 & 17.7 & * \\
\hline
\end{tabular}

\begin{tabular}{|c|c|c|c|c|c|c|c|c|c|}
\hline \multirow[b]{2}{*}{$K$ values } & \multicolumn{5}{|c|}{$X \longrightarrow M$} & \multicolumn{4}{|c|}{$M \longrightarrow \Gamma$} \\
\hline & 0.10 & 0.20 & 0.40 & 0.80 & 1.00 & 0.05 & 0.10 & 0.20 & 0.60 \\
\hline Lower Boundary & 17.90 & 17.66 & 17.06 & 16.13 & 16.03 & 14.74 & 13.50 & 11.06 & 3.06 \\
\hline Bound States & & & & & & & & & \\
\hline$G(0,0)$ & no & no & no & no & no & 14.61 & 13.45 & $*$ & $*$ \\
\hline$G(1,1)$ & no & no & no & no & no & 14.61 & 13.45 & $*$ & * \\
\hline$G\left(1^{\prime}, 1^{\prime}\right)$ & $*$ & 17.5 & 16.7 & 15.5 & 15.33 & 14.61 & 13.45 & $*$ & $*$ \\
\hline
\end{tabular}

terminology [23] to call the bound state where all three Green's functions have poles as type-S exchange bound state, and the bound state where only $G\left(1^{\prime}, 1^{\prime}\right)$ has pole as type-D. So in the absence of anisotropy only type-S bound state exists in $\Gamma \longrightarrow X$ and $M \longrightarrow \Gamma$ regions, while only type-D bound state exists in $X \longrightarrow M$ region.

The enhancement of weight at the bottom of continuum of all $G(0,0), G(1,1), G\left(1^{\prime}, 1^{\prime}\right)$ (Figure 4.4) are found relatively strong at the point $K=0.8$ of the distance from $\Gamma$ towards $X$, but dissappear right after this point. At the same time the type-S bound state emerge in the higher $K$ interval. This indicates that some weight transfers from the continuum as a discrete bound state. The transfer of weight from continuum to bound state is more visibly illustrated in $X \longrightarrow M$ region, where a sharp peak 
of each of $G(1,1)$ and $G\left(1^{\prime}, 1^{\prime}\right)$ is found very close to the bottom of continuum at $K=0.1$ of the distance from $X$ towards $M$. Then the type-D bound state, which is the only bound state of $G\left(1^{\prime}, 1^{\prime}\right)$ leaves the continuum. Afterward at $K=0.4$ only a strong enhancement of $G(1,1)$ is found at the bottom of continuum, while the weight of $G\left(1^{\prime}, 1^{\prime}\right)$ probably goes into this bound state. Both $G(0,0), G(1,1)$ keep strong enhancement at the lower boundary of continuum until $\vec{K}=(0, \pi)$ (M-point), where they leave the bottom and each forms another resonance.

However our picture of bound states seems contrary to what people usually expect. It was widely believed that the number of bound states existing at the zone corner depends on the dimension of lattice $n$. So two distinct branches of bound states were expected for all 2-dimensional lattices [27]. Our study shows that, while it is true in the simple cubic ferromagnet, it is not always necessarily the case in complicated lattices, such as the 2-d triangular lattice. Instead, the parameters as S, $\sigma$ and D all have an effect on the picture.

The effect of the spin magnitude $S$, which have not always been studied by other researchers, is found to be quite significant to the behaviour of bound states. Figure 4.5 shows this interesting discovery. For $S=1$ in the absence of anisotropy the type-D branch is merged by the continuum between $\Gamma \longrightarrow X$ and type-S bound state is merged by the continuum between $X \longrightarrow M$. So only one bound state appears at each $\vec{K}$ value. When the $S$ changes from 1 to $\frac{1}{2}$ while other parameters are kept the same, the bound state are more strong bound so that both of type-S and type-D bound states appear for $S=\frac{1}{2}$, in agreement with Wada etal[27]. 


\section{BOUND STATES DISPERSIONS FOR $S=1$ AND $S=1 / 2$ $(\sigma=1.0 . D=0.0 . T-X-M)$}

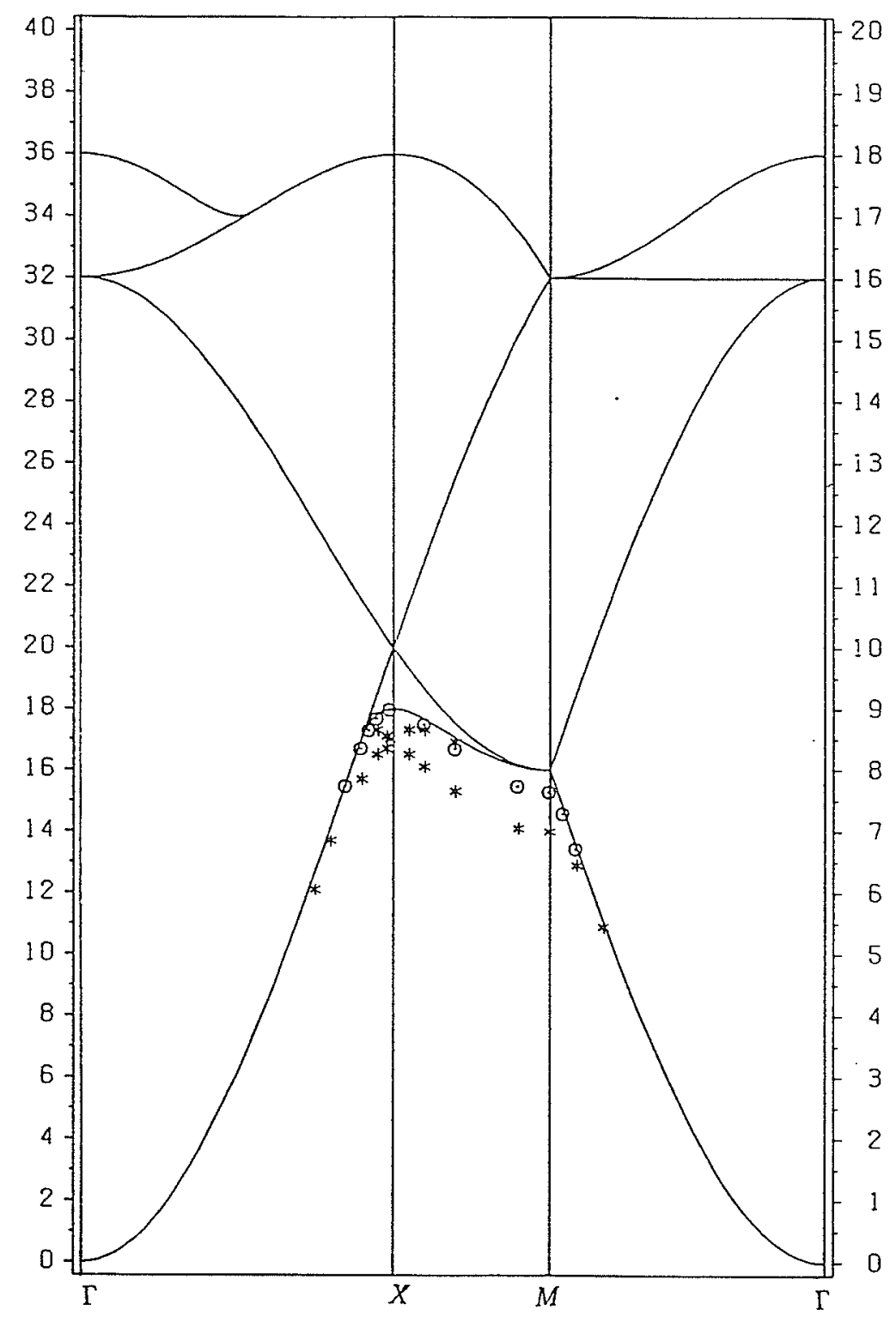

$\odot--$ BOUND STATES FOR $S=1$
--- BOUND STATES FOR $S=1 / 2$

Figure 4.5: Dispersion of Bound States for $S=1$ and $S=\frac{1}{2}$ 
Wada etal have examined the bound states of 2 -d triangular lattice for $S=\frac{1}{2}$ by another method, which is also described in Mattis's book[3]. In order to verify our results, we programmed that formulae and recalculated the bound states at both $S=1$ and $S=\frac{1}{2}$, and carefully compared the data from two methods. All results reached full agreement within the resolution of their published diagrams.

\subsection{Two-Magnon spectrum with Various Anisotropies}

Before we examine the influence of the Ising $(\sigma)$, and uniaxial $D$ anisotropies on the continuum, we shall first briefly discuss the Ising levels. If the Hamiltonian takes the form of equation (1.2), the Ising level energy ( $\operatorname{set} J_{i j}=0$ ) is given by,

$$
H=-\sum_{i j} I_{i j} S_{i}^{z} S_{j}^{z}-\sum_{i} D\left(S_{i}^{z}\right)^{2}
$$

The ground state is,

$$
H_{\text {ground }}=-\sum_{i j} I_{i j} S^{2}-N D S^{2}
$$

Then when two spins are turned over on the same site $(m)$, eq. (4.1) gives,

$$
H=-\sum_{i j} I_{i j}\left(-S+2 \delta_{i m}\right)\left(-S+2 \delta_{i m}\right)-\sum_{i} D\left(-S+2 \delta_{i m}\right)^{2}
$$

Therefore for $S>1 / 2$ and only considering the nearest-neighbour interaction, the single-ion Ising level is given by,

$$
E_{00}=H-H_{\text {ground }}=4 S \sum_{i} J_{i}=24 S J_{1}
$$

For two spins being turned over on two different $\operatorname{sites}(m$ and $l)$,

$$
H=-\sum_{i j} I_{i j}\left(-S+\delta_{i m}+\delta_{i l}\right)\left(-S+\delta_{i m}+\delta_{i l}\right)-\sum_{i} D\left(-S+\delta_{i m}+\delta_{i l}\right)^{2}
$$


When $l$ and $m$ are adjacent, the NN Ising level is,

$$
\begin{aligned}
E_{11}=H-H_{g r o u n d} & =4 S \sum_{i} I_{i}-2 I_{l m} S-2 D(-2 S+1) \\
& =22 S I_{1}+2 D(2 S-1)
\end{aligned}
$$

So an increase of single-ion anisotropy $D$ will cause the NN Ising level to rise or even cross over the single-ion Ising level, which is, instead, unaffected by $D$. In Figure 4.2 we set $\sigma=0.1$, illustratively $G(0,0), G(1,1)$ both become a $\delta$-function-like spectrum located at their corresponding Ising level positions.

Three different situations of anisotropies are studied. D- and $\sigma$-anisotropies are switched on respectively in Figure 4.6 and Figure 4.7. In Figure $4.8 D=2.26$ and $\sigma=0.78$ are chosen to compare with the Raman scattering experiment of one- and two- magnon excitations in $\mathrm{Fe} B r_{2}$, which will be briefly discussed in the last section of this chapter. From Figures 4.1 to 4.7 the NN Ising level increases by $2 D=4.52$, we do observe the corresponding resonance of $G(1,1)$ at $\vec{K}=0$ also increase from 30.8 to 35.5 by 4.7 . Similiarly from Figure 4.6 to 4.8 it increases from 28.40 to 33.10 by 4.7. At the same time the peak of $G(0,0)$ remains almost unchanged.

On closer inspection of the spectrum at $\vec{K}=0$ we find that the resonance of $G(0,0)$ is not strongly affected by $D$-anisotropy even when $D$ produces a gap below the continuum and lifts the whole band of the continuum up (the process shown from Figure 4.1 to 4.7 , and from Figure 4.6 to 4.8 ), but this resonance is shifted slightly when $\sigma$-anisotropy squeezes the band (the process shown from Figure 4.1 to 4.6, and from Figure 4.7 to 4.8 ). On the other hand, the $G(1,1)$ resonance moves more strongly following the shift and deformation of the band when the band is lifted up 
by $D$ and squeezed by $\sigma$, but the position of the resonance with respect to the band remains relatively unchanged.

Figures 4.9, 4.10 and 4.11 respectively show the dispersions of bound states and resonances for the anisotropic situations mentioned above. Then Figures 4.12, 4.13, 4.14 respectively show the spectrum of two-magnon propagators for selected $\vec{K}$ values. Comparing these figures with the isotropic Figures 4.3, 4.4 we find the critical lines still play an important role in the evolutions of two-magnon spectrum with various anisotropies. Critical lines still divide the weight of spectrum into "main peaks" and "secondary humps", and still to some extent direct the evolutions of resonances. Most of our discussion of isotropic figures are still useful, but there are some significant differences due to the effect of anisotropies which one can easily see from Figures 4.6 to 4.14. The trajectory of the positions of resonances are apparently shifted, mainly due to the shift of their starting points as we discussed in previous paragraph. Another phenomena is that the humps formed on the upper side of critical line become much smaller as D becomes larger. From our studies of the pictures of four different anisotropic cases, it appears that the evolution of resonances probably can be roughly determined by knowing their starting positions and the structures of the pictures of critical lines.

Figures 4.9, 4.10 and 4.11 also illustrate the effects of various anisotropies on the behaviour of bound states. It shows that the type-D exchange bound states are relatively unaffected by the presence of uniaxial-anisotropy though they seem to be 'pushed' closer to the bottom of continuum in Figure $4.10 \&$ 4.11. The type-S branch 


\section{TWO MAGNON PROPAGATORS IN 2-D TRIANGULAR LATTICE} $($ SIGMA $=J i / 11=0.78, S=1, D=0.0, K=0.0$ )

$$
\begin{aligned}
& -I M(G(0,0)) \\
& -I M(G(1,1))
\end{aligned}
$$

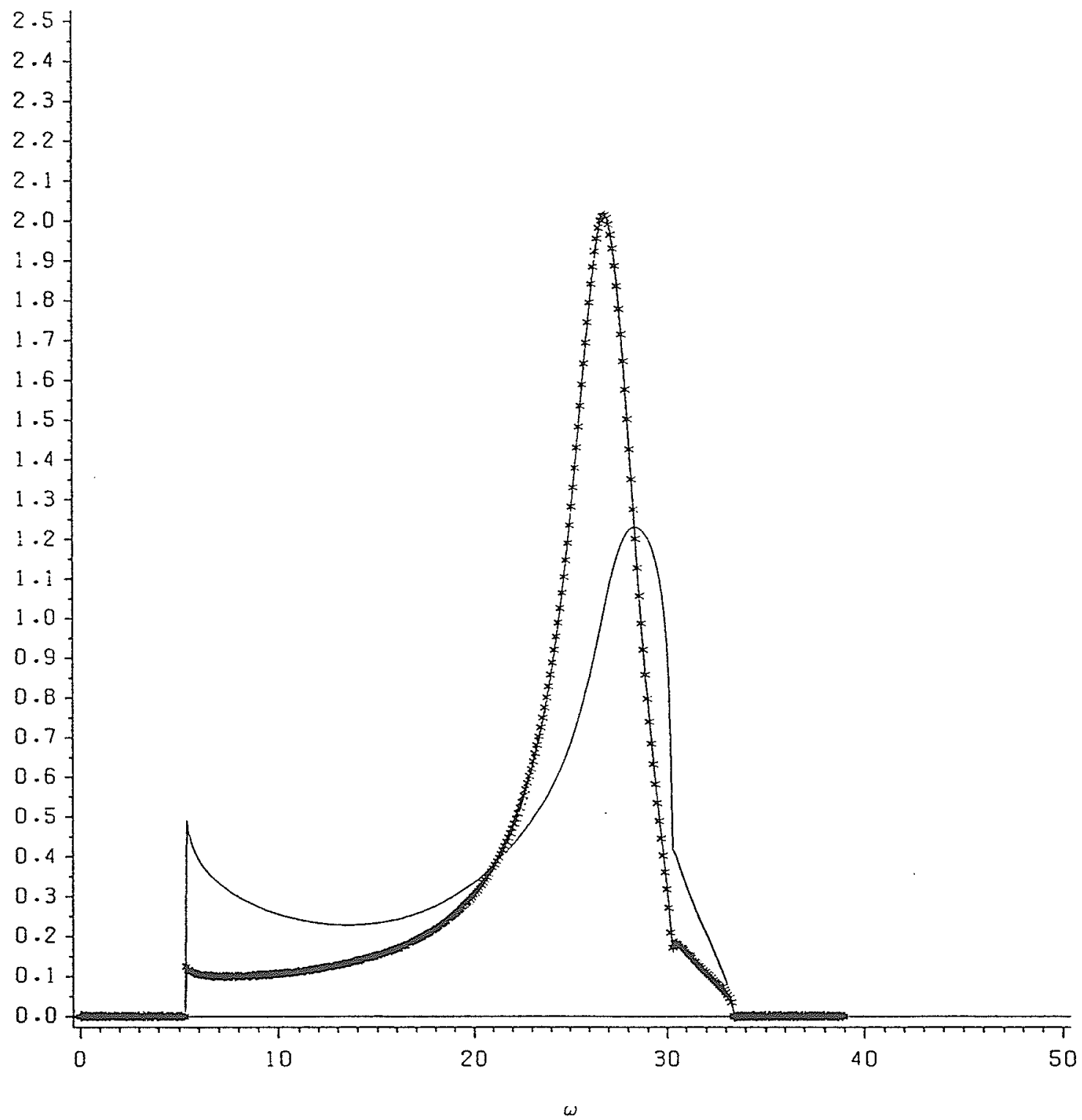

$$
\begin{aligned}
& \text { STAR LINE }-\cdots--\operatorname{lm}(G(0.0)) \\
& \text { SOLID LINE }-\cdots-1 \mathrm{~m}(G(1.1))
\end{aligned}
$$

Figure 4.6: Two-magnon spectra at $K=0$ for $D=0, \sigma=0.78$ 


\section{TWO MAGNON PROPAGATORS IN 2-D TRIANGULAR LATTICE $($ SIGMA $=J 1 / 11=1.0, S=1, D=2.26, K=0.0)$}

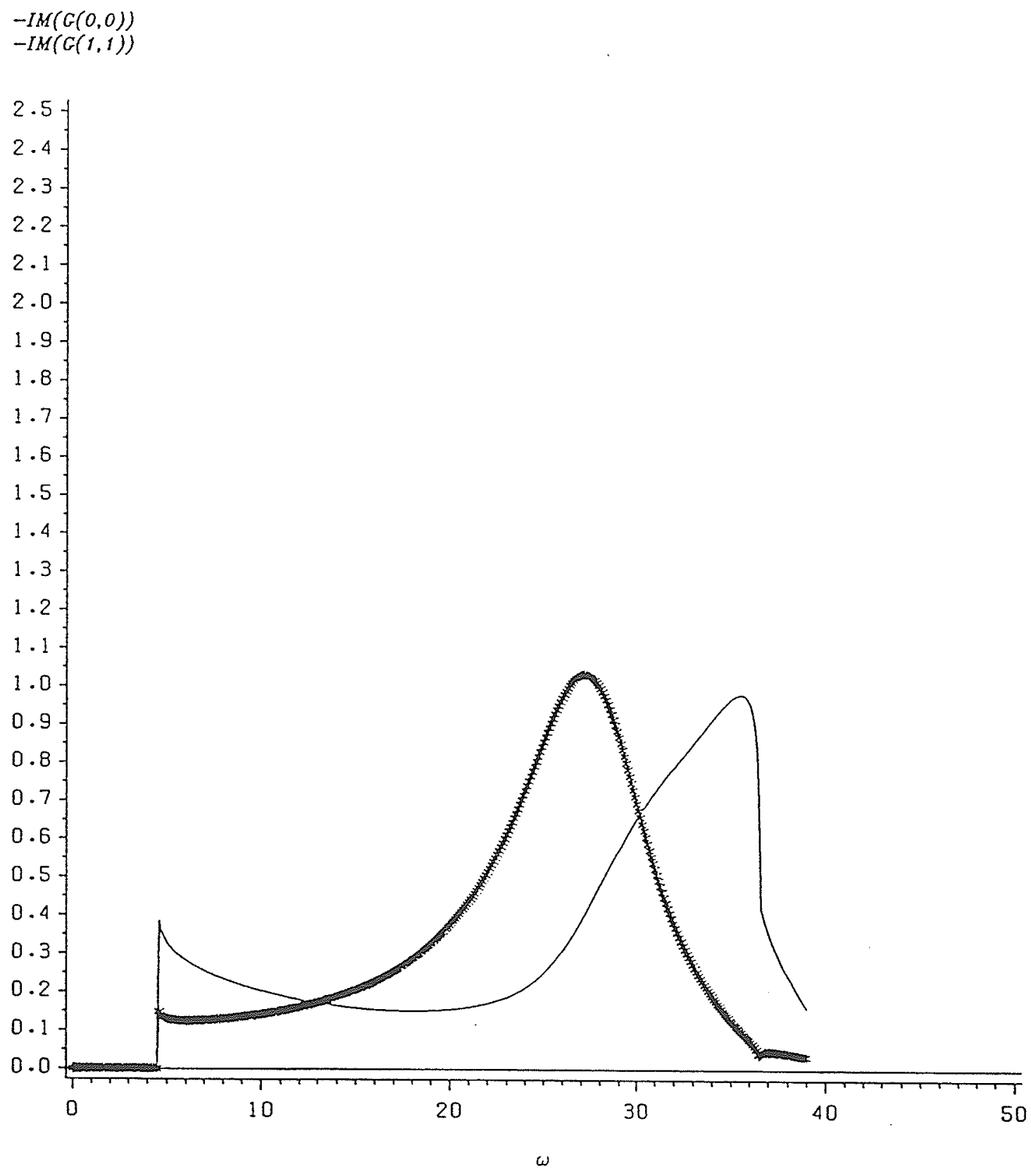

STAR LINE $-\cdots--\operatorname{lm}(G(0.0))$
SOLID LINE $----\operatorname{lm}(G(1.1))$

Figure 4.7: Two-magnon spectra at $K=0$ for $D=2.26, \sigma=0.0$ 
TWO MAGNON PROPAGATORS IN 2-D TRIANGULAR LATTICE $(\operatorname{SIGMA}=31 / 11=0.78 . S=1, D=2.26 . K=0.0)$

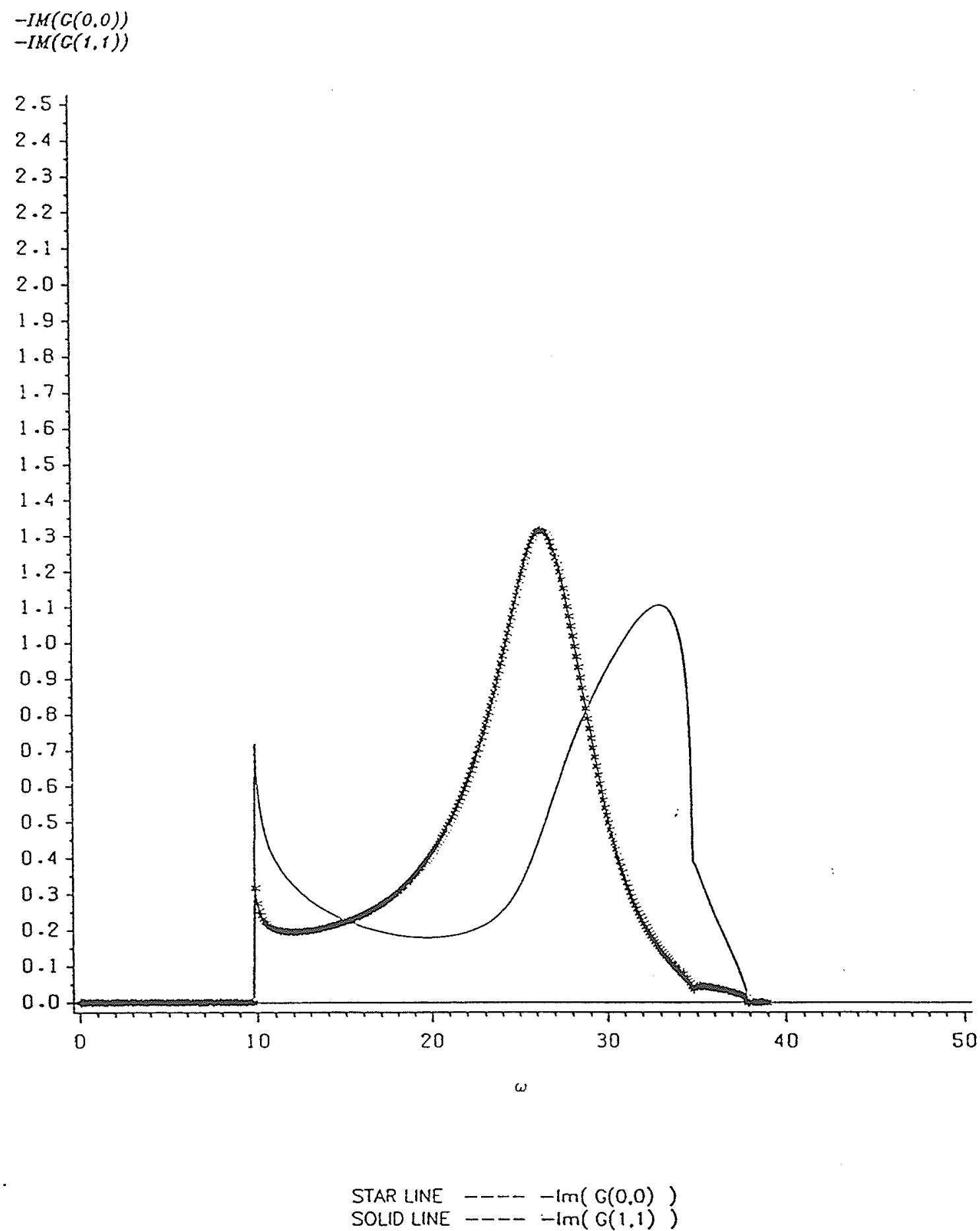

Figure 4.8: Two-magnon spectra at $K=0$ for $D=2.26, \sigma=0.78$ 


\section{TWO-MAGNON DISPERSION RELATION $(S=1, \sigma=0.78 . \quad D=0.0)$}

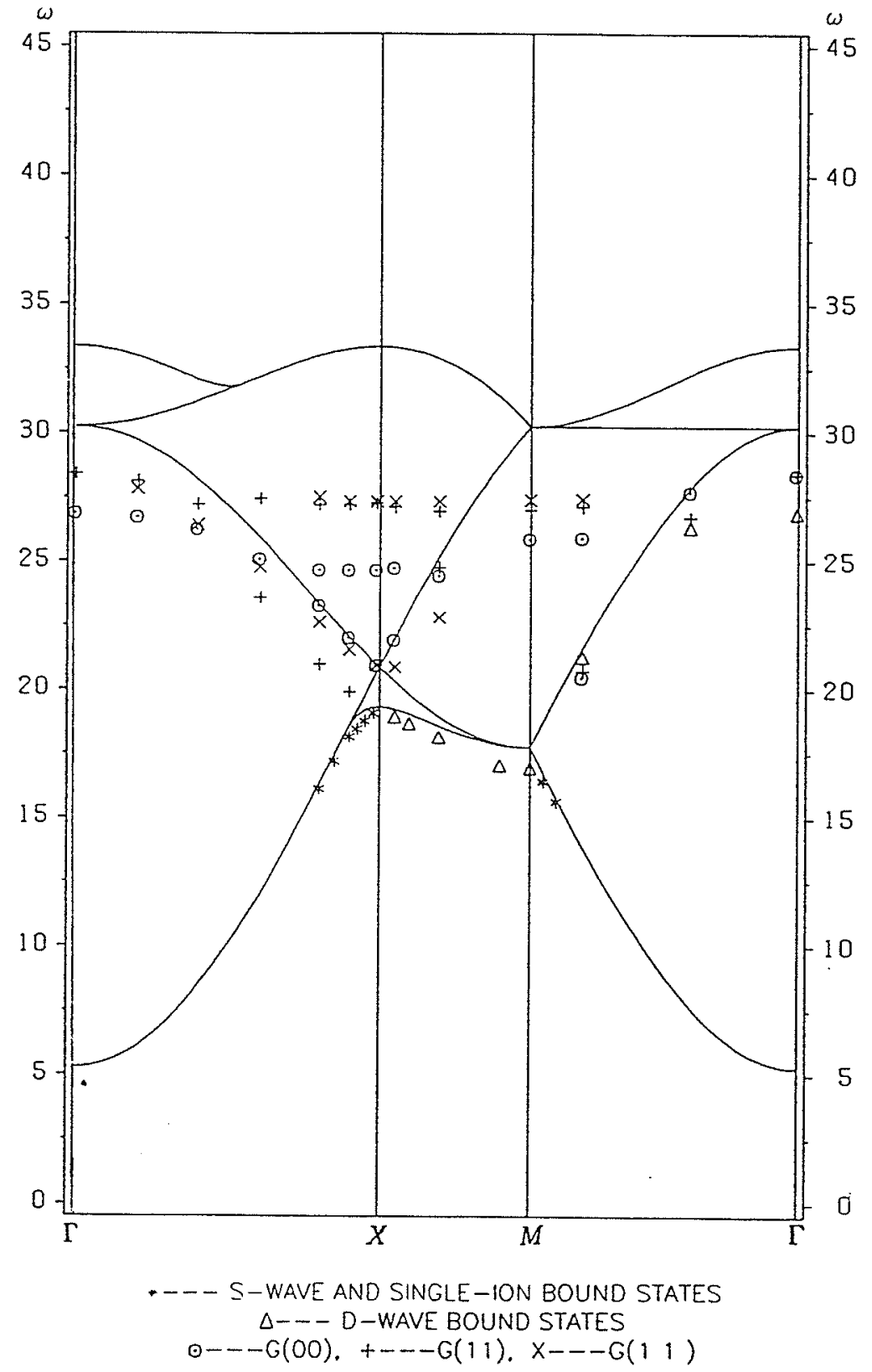

Figure 4.9: Dispersion of resonance peaks and bound states for $S=1$ and $D=0, \sigma=0.78$ 


\section{TWO-MAGNON DISPERSION RELATION $(S=1 . \sigma=1.0, D=2.26)$}

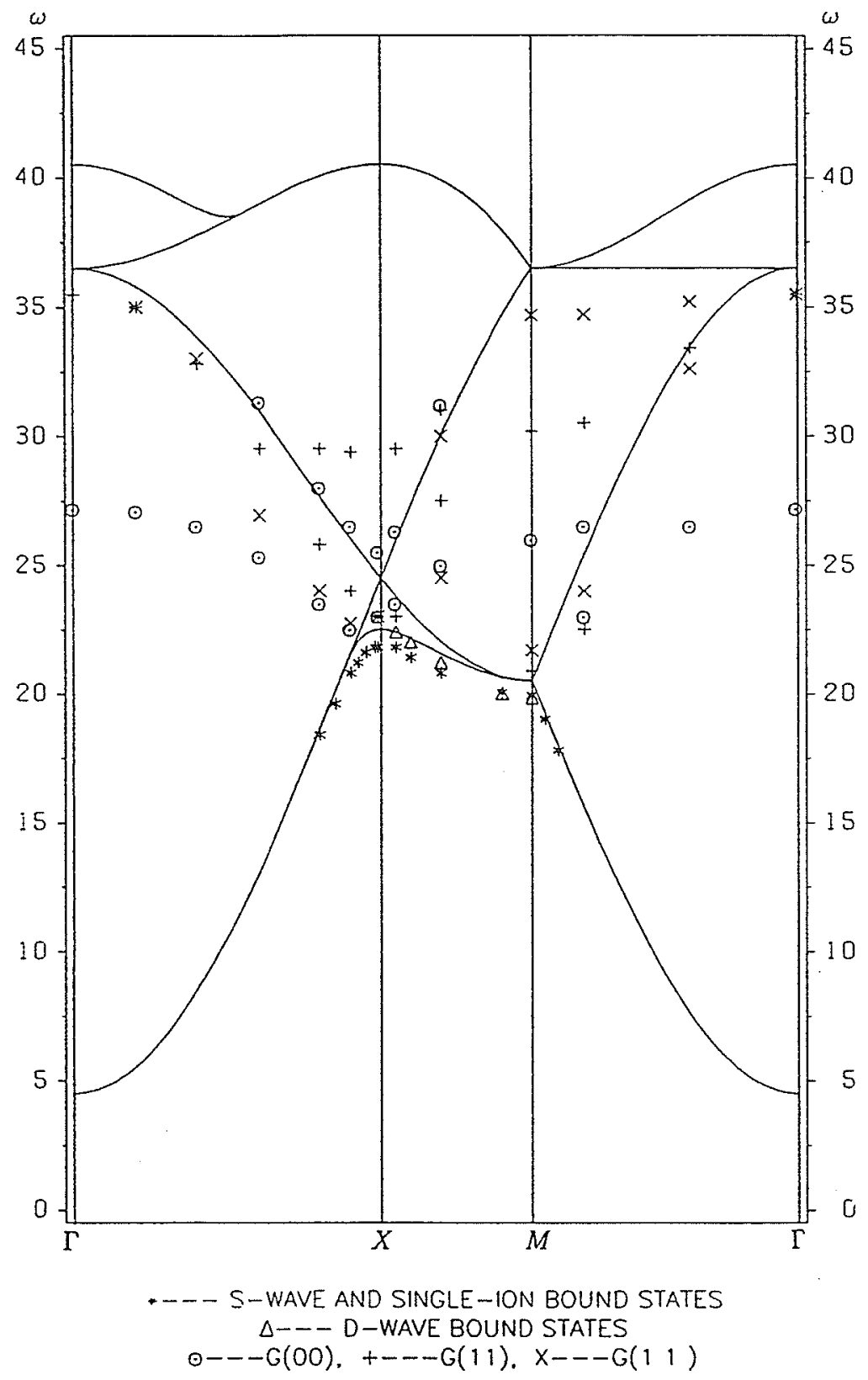

Figure 4.10: Dispersion of resonance peaks and bound states for $S=1$ and $D=2.26, \sigma=1.0$ 


\section{TWO-MAGNON DISPERSION RELATION $(S=1, \sigma=0.78 . \quad D=2.26)$}

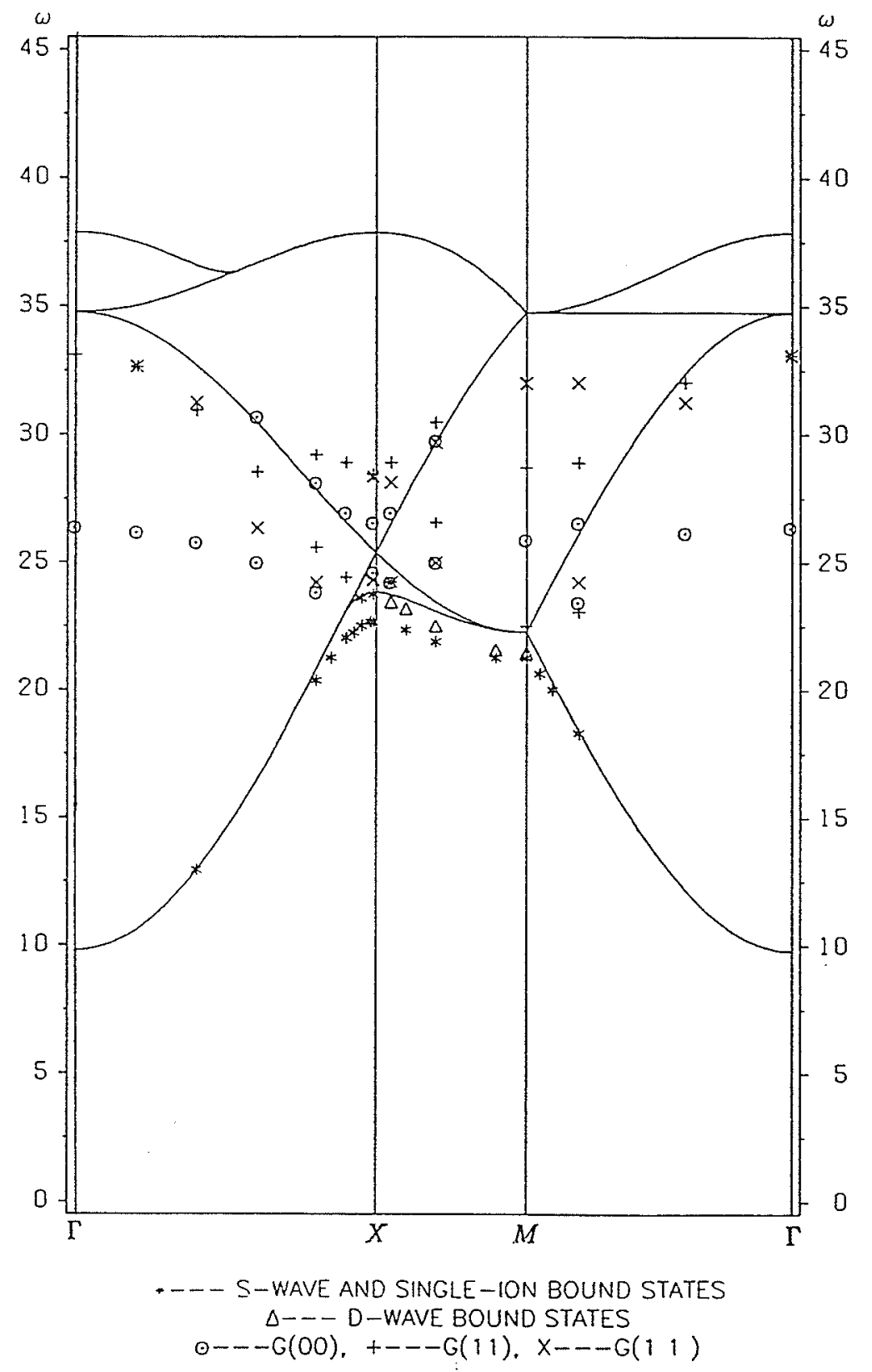

Figure 4.11: Dispersion of resonance peaks and bound states for $S=1$ and $D=2.26, \sigma=0.78$ 


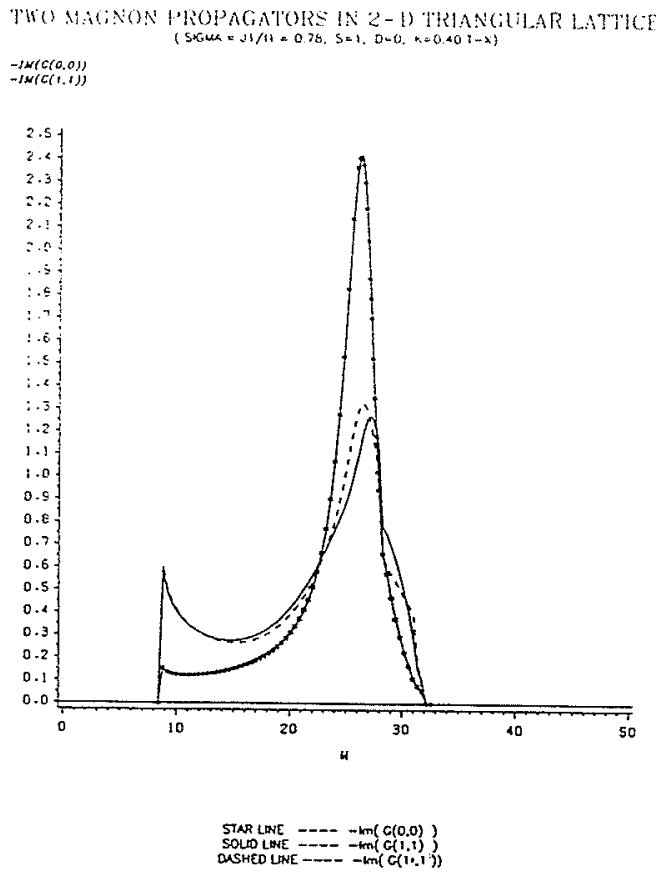

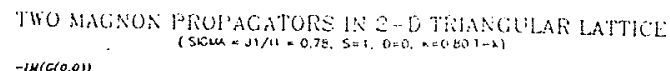
$-i n(c(0.0))$
$-1 \mu(c(t, 1))$
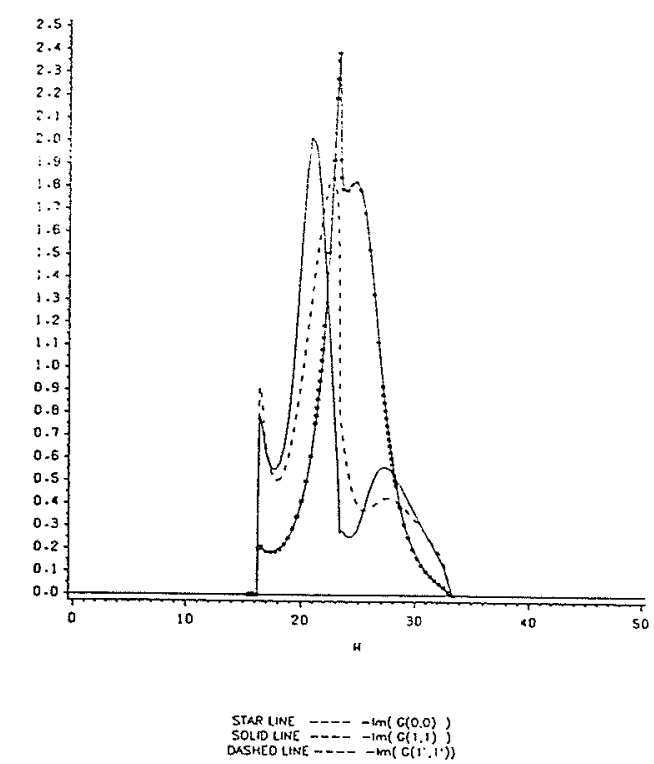

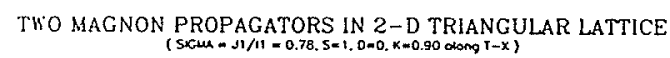

TWO MAGNON PROPAGATORS IN 2-D TRIANGULAR LATTICE

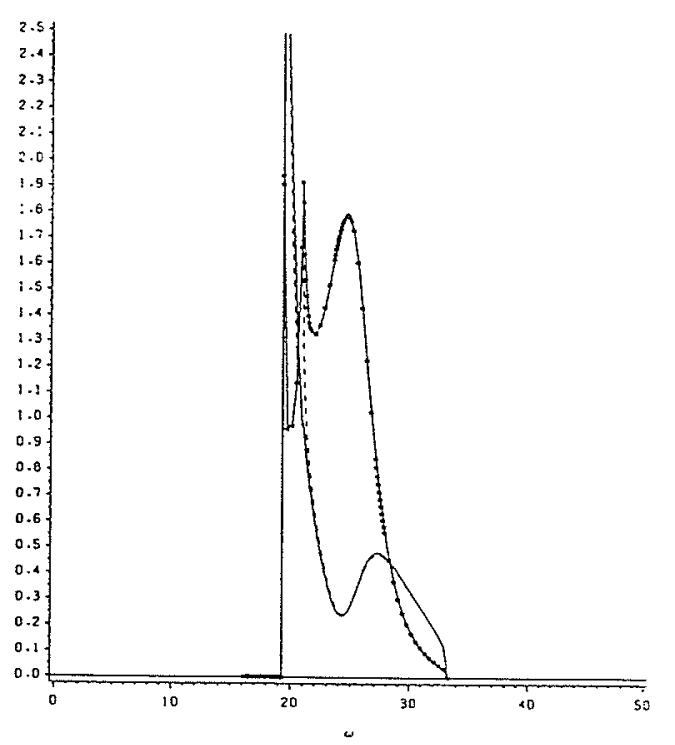

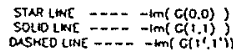

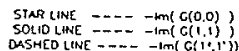

Figure 4.12: The evolution of two-magnon propagators for $D=0.0, \sigma=0.78$ 


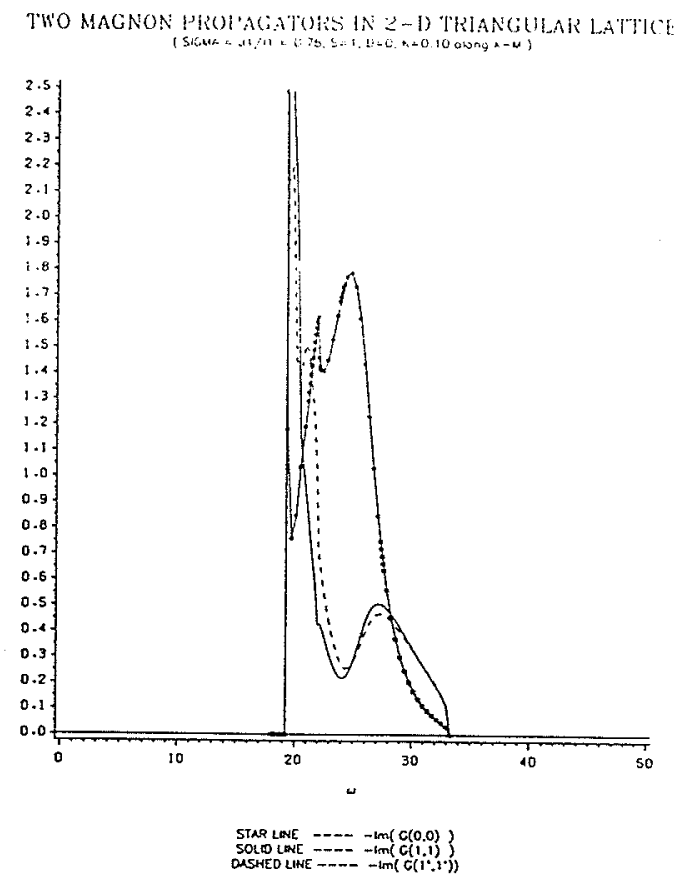

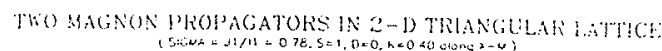

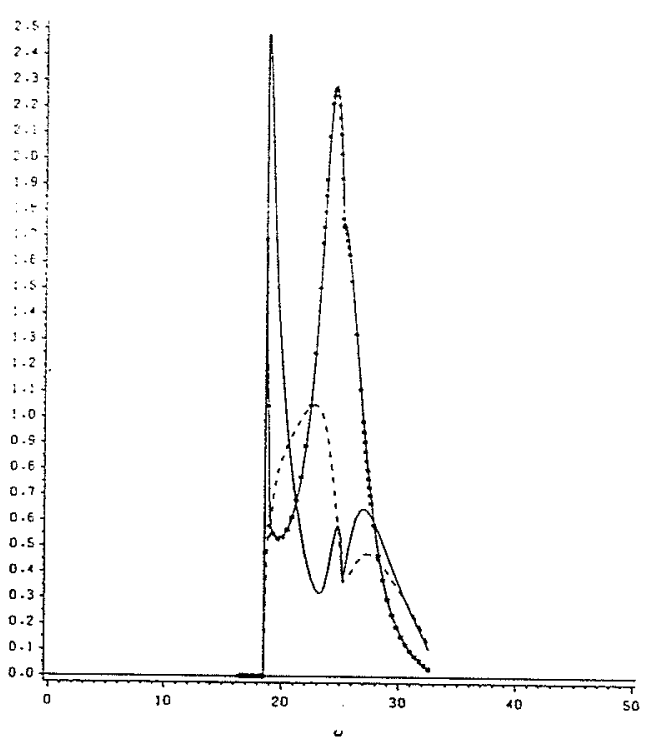

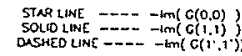

TWO MAGNON PROPAGATORS IN 2-D TRIANGULAR LATTICE
$(5 \times 4,=51 / 11=0.78,5=1,0=0.4-$ PONI $)$

TWO MACNON PRUPAGATORS IN $2-$ D TRIANGULAR LATTICE

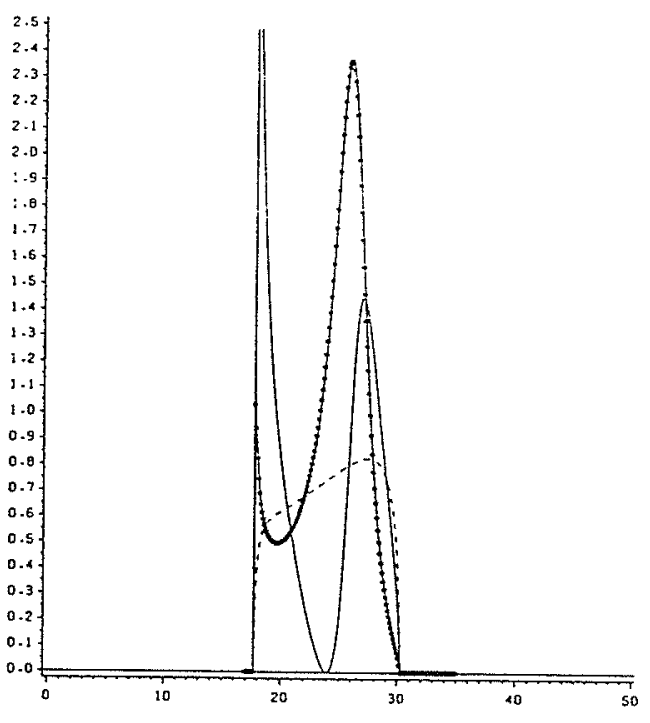

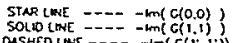

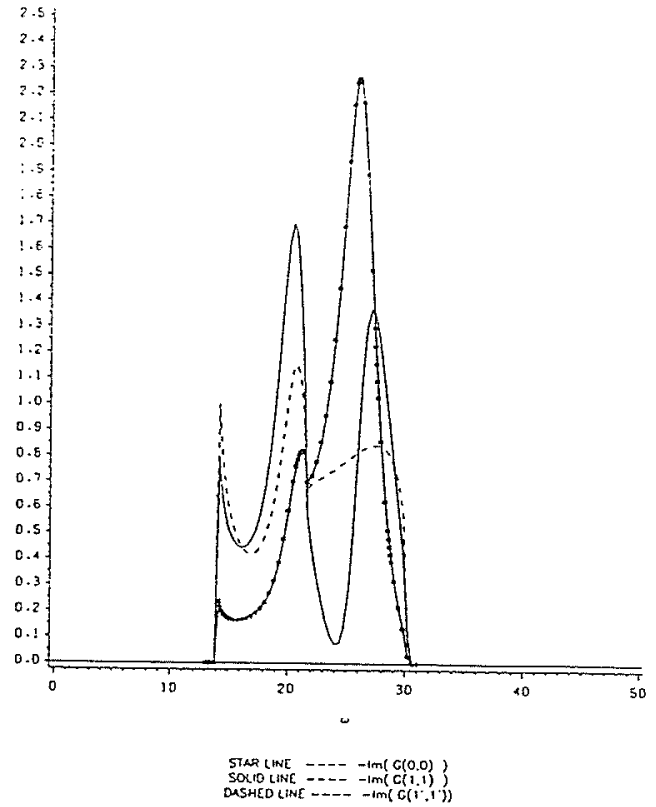



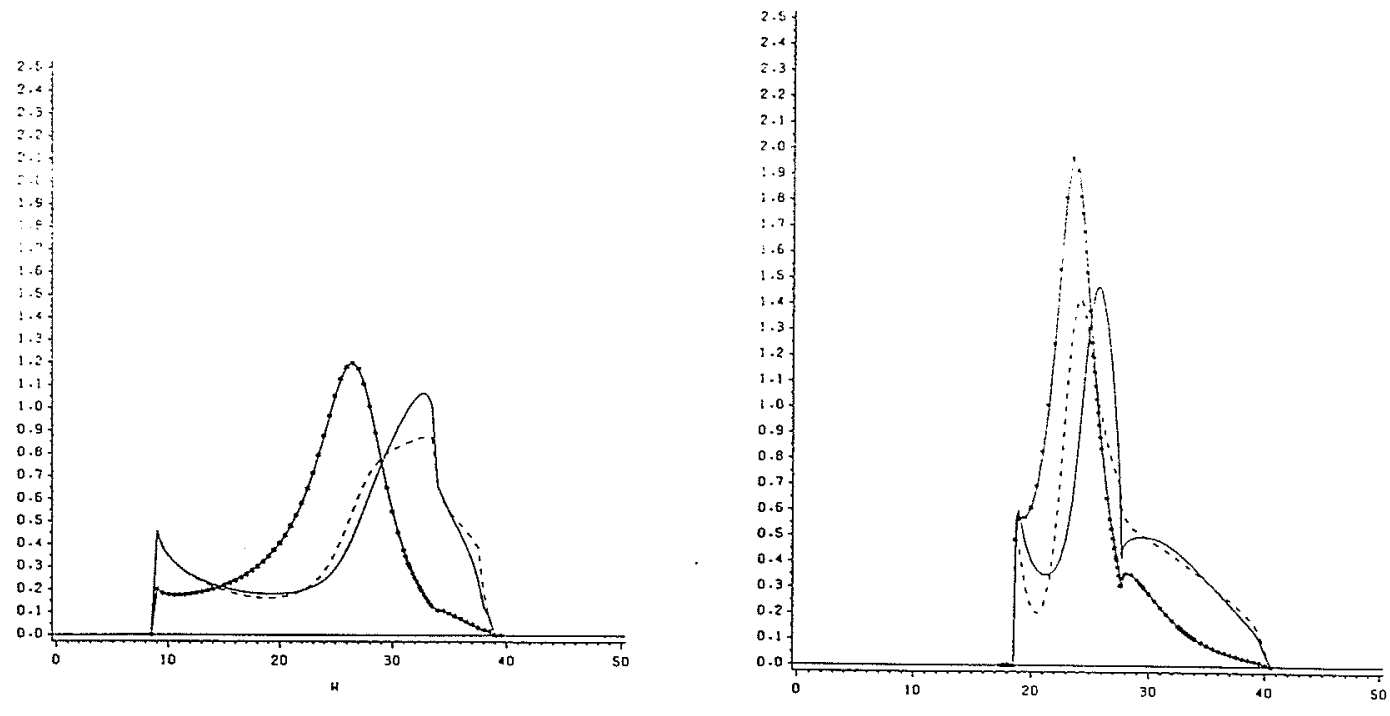

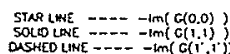

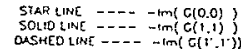

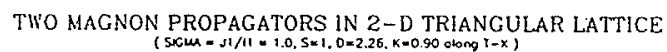

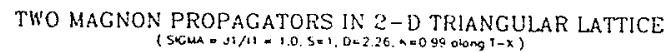

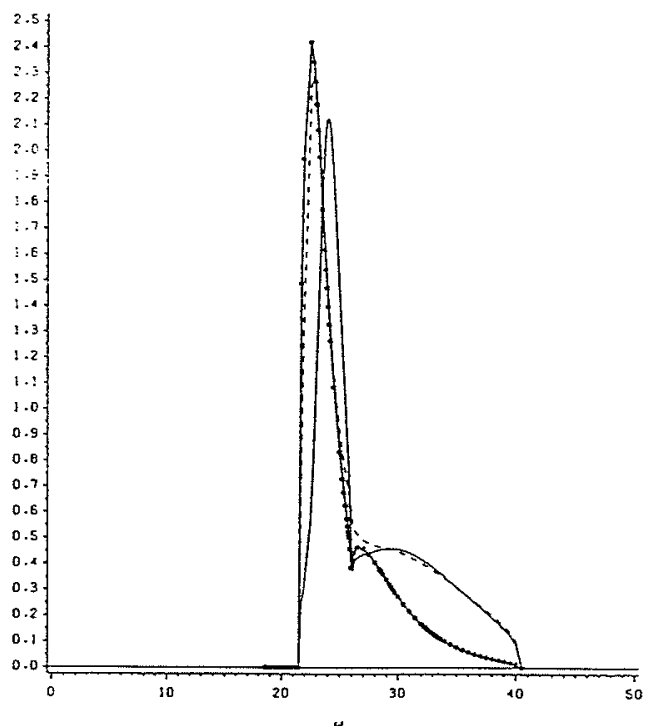

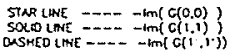

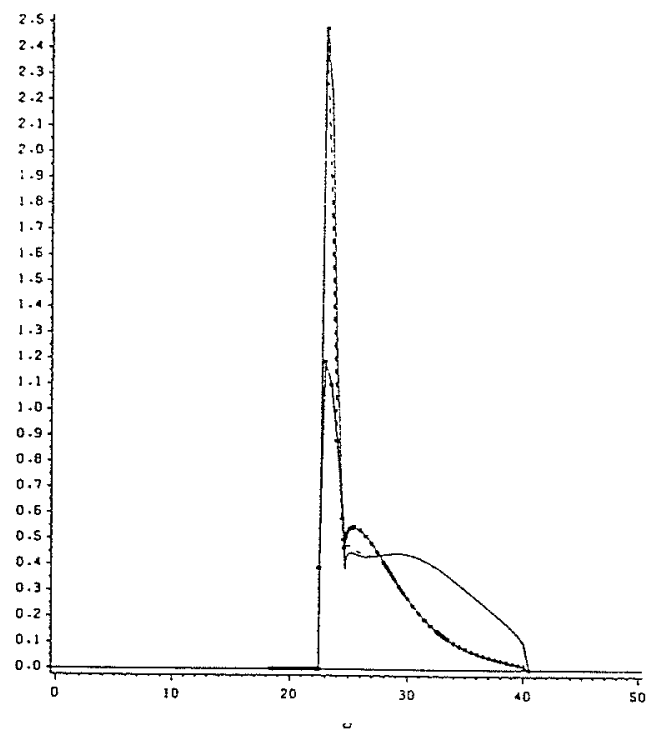

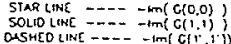

Figure 4.13: The evolution of two-magnon propagators for $D=2.26, \sigma=1.0$ 


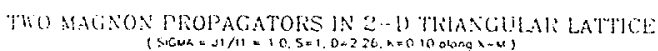

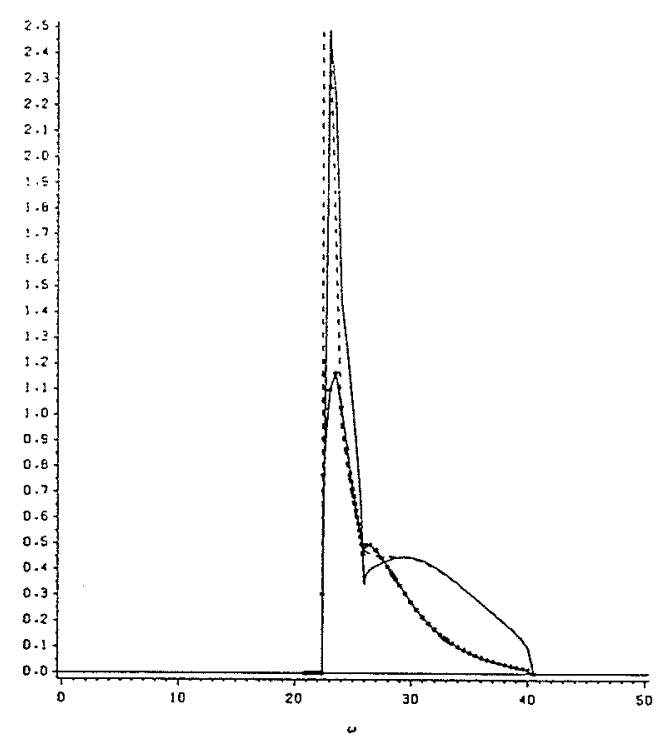

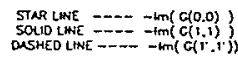

TWO MAGNON PROPAGATORS IN 2-D TRIANGULAR LATTICE

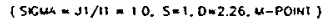

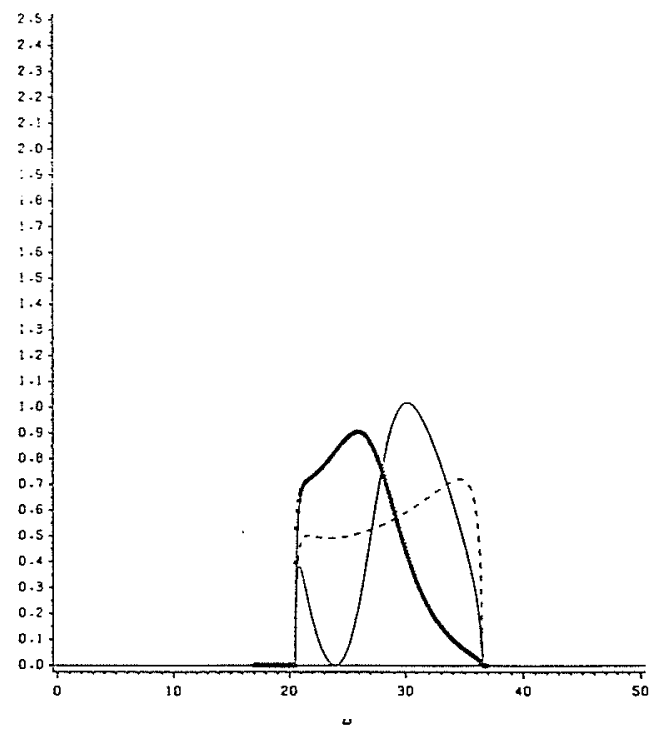

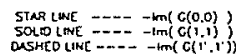

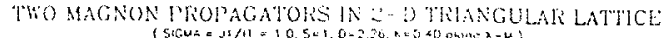

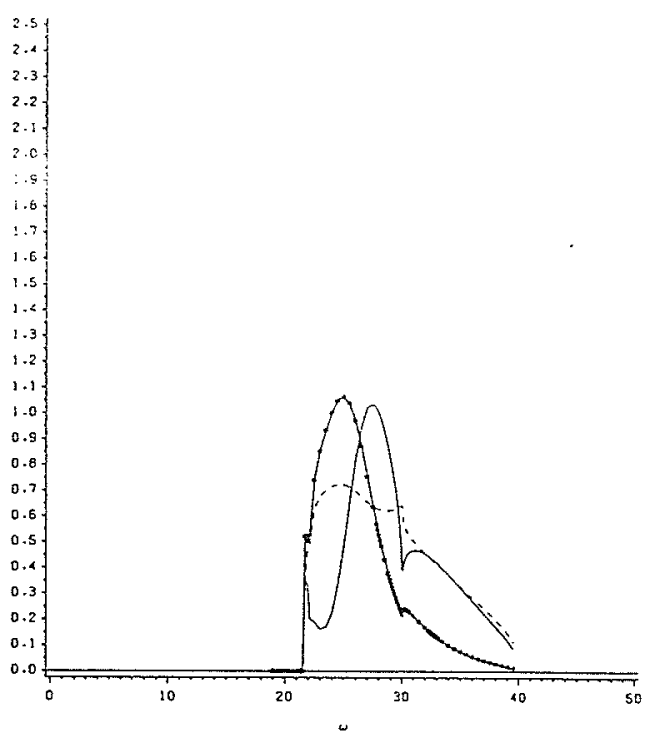

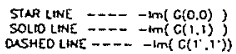

TWO MAGNON PROPAGATORS IN $2-1)$ TRIANGULAR LATTICE

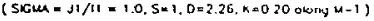

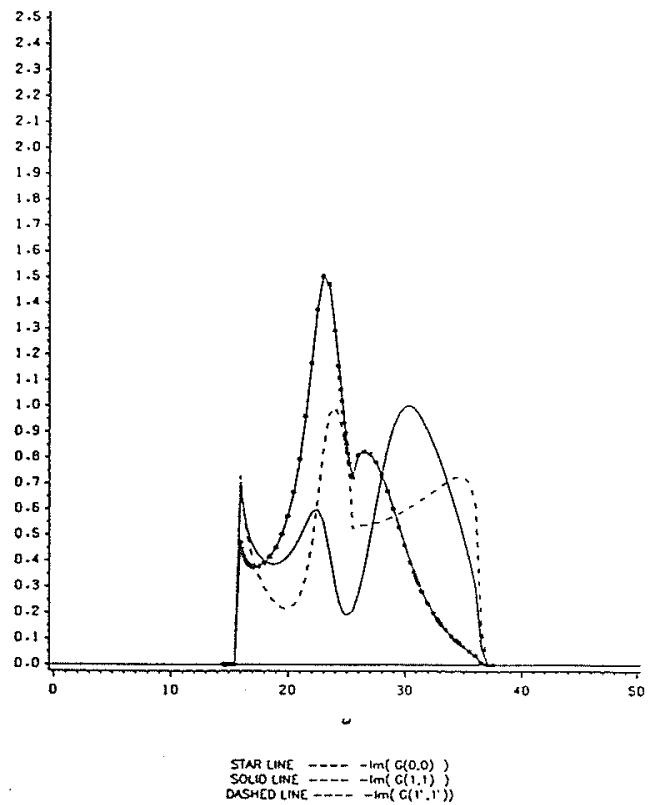



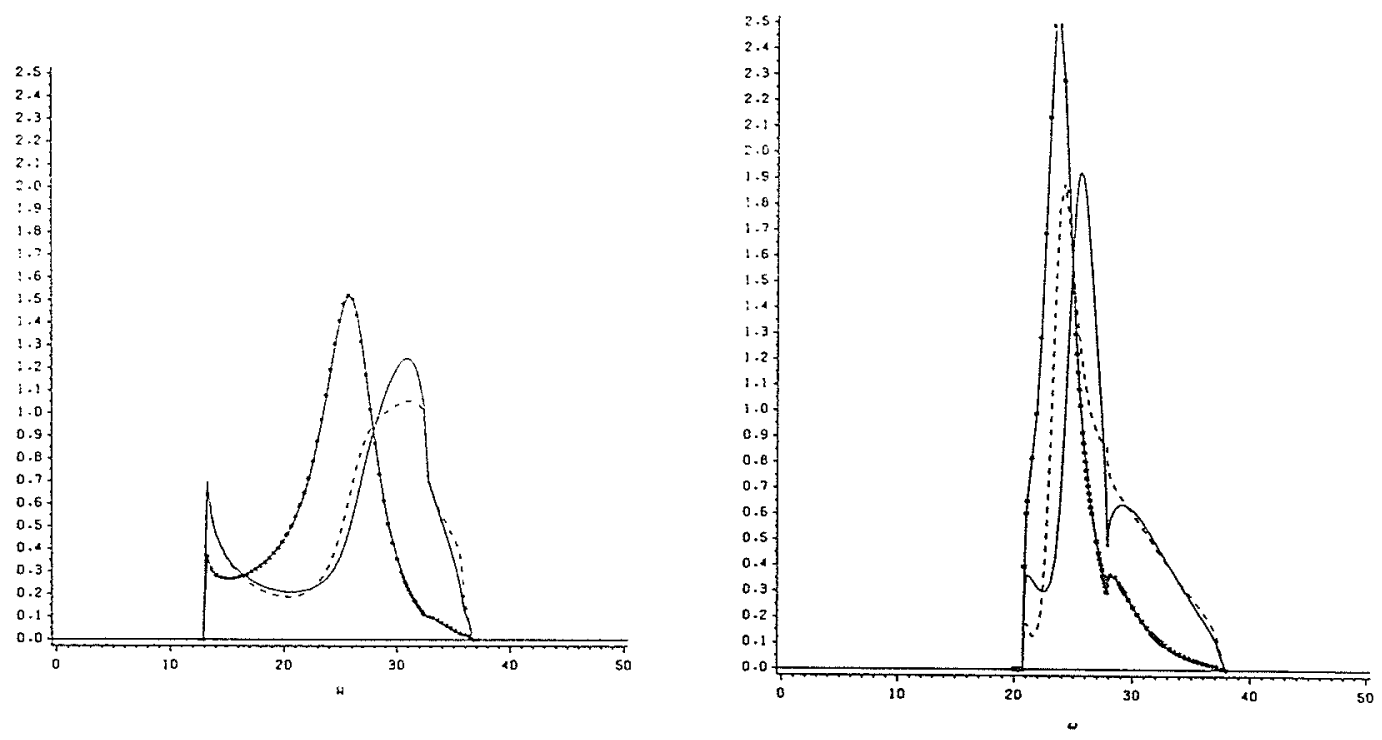

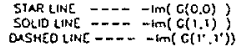

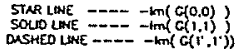

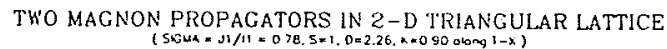

TWO MAGNON PROPAGATORS IN 2-D TRIANGULAR LATTICE

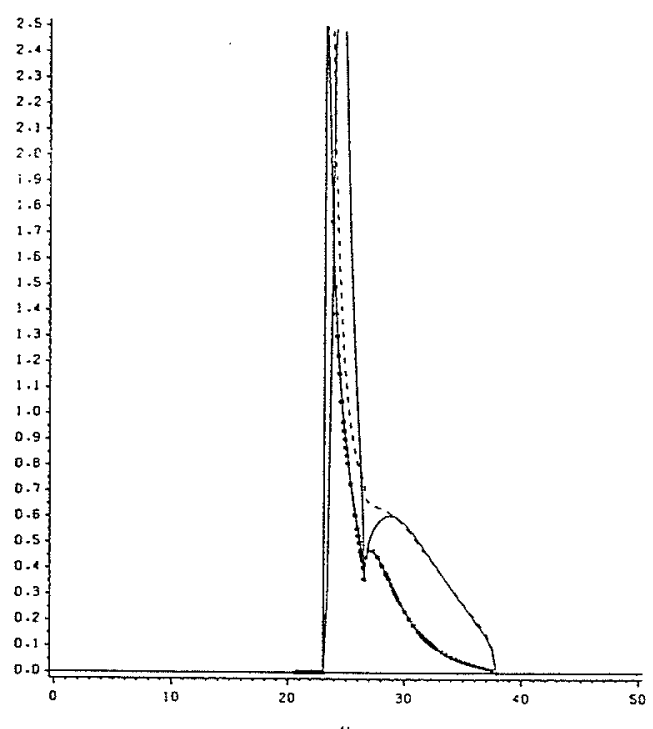

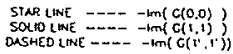

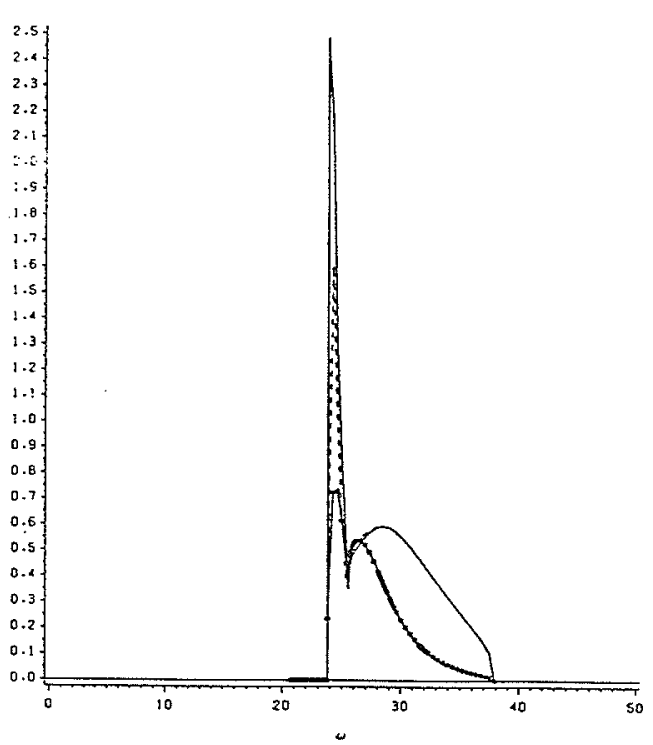

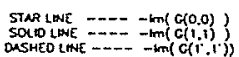

Figure 4.14: The evolution of two-magnon propagators for $D=2.26, \sigma=0.78$ 
THO MACONON PROPAGATOKS IN 2-D TRIANGULAR LATTIC:

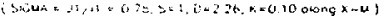

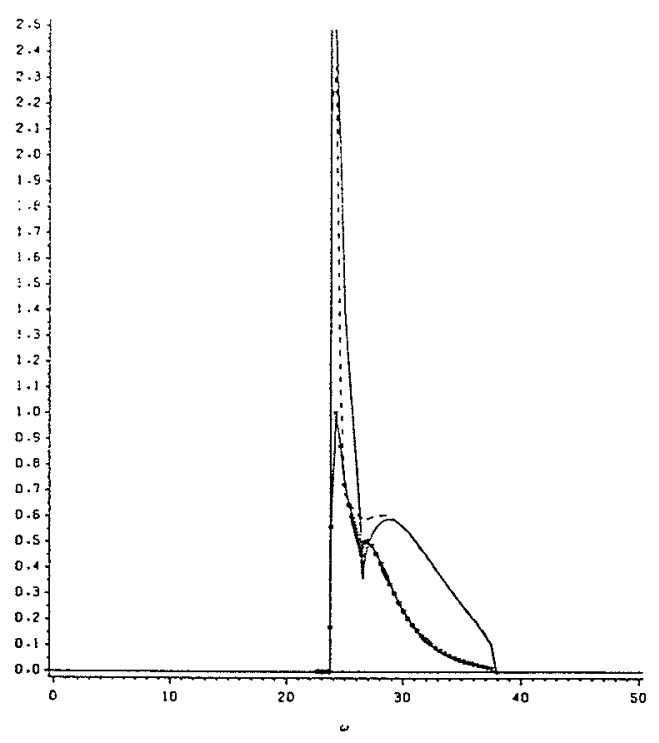

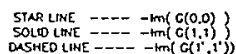

TWO MAGNON PRUPAGATORS IN 2-D TRIANGULAR LATTICE

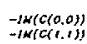

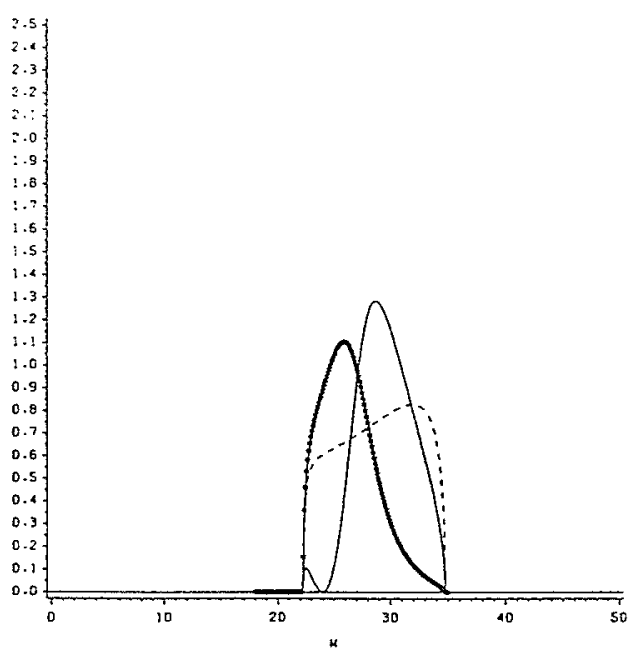

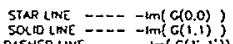

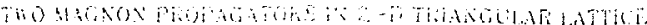

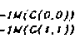

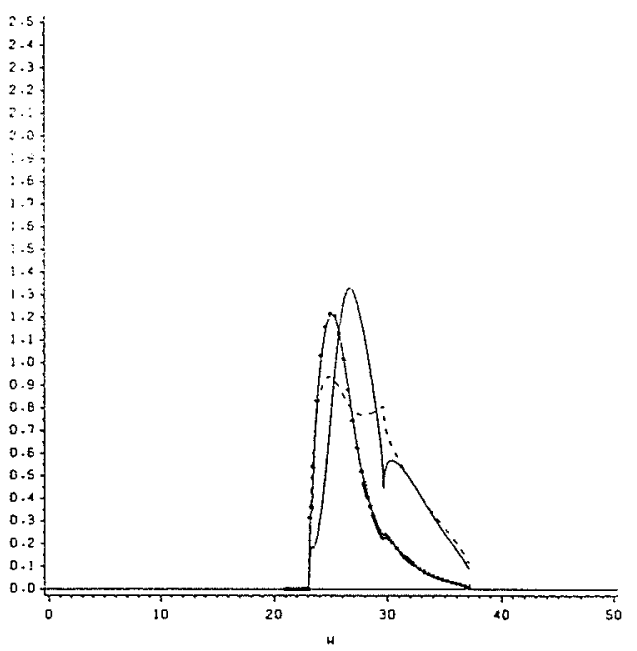

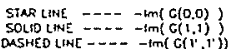
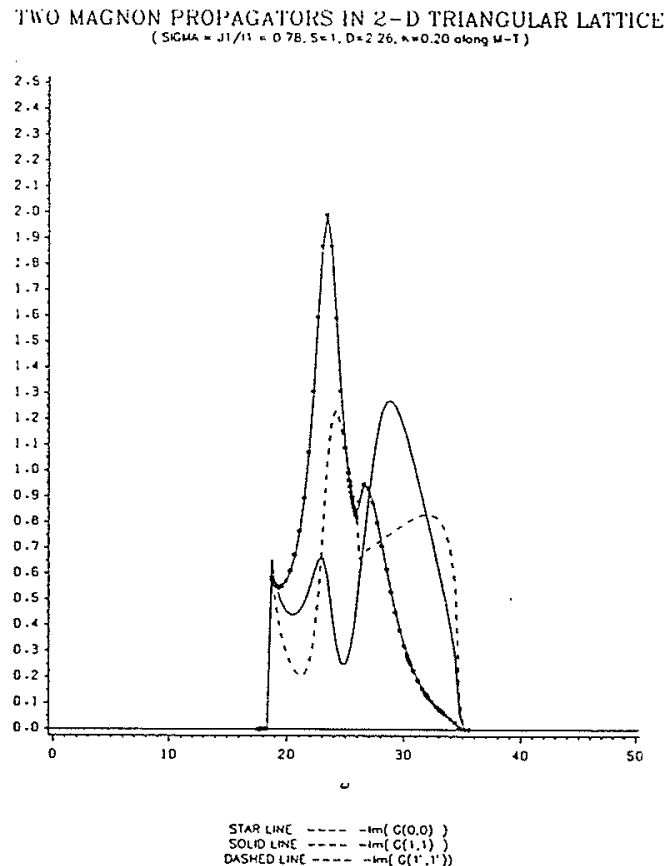
is affected in a similiar way. Since it is originally pretty close to the continuum, it is even "pulled in" in Figure 4.10 and only reappears in Figure $4.11(\sigma=1.0, D=2.26)$ when $\sigma$ changes from 1.0 to 0.78 .

However the most significant change is the appearance of an additional 'single-ion' bound states(Figure $4.10 \& 4.11$ ) obviously due to the effect of $D$-anisotropy. For the values of $D$ used it exists below both of type-S and type-D bound states in all regions $\Gamma \longrightarrow X \longrightarrow M \longrightarrow \Gamma$. We notice that the single-ion bound states are even

found for some small $|\vec{K}|$ values. We also suspected its existence at $\vec{K}=0$, though we didn't find it in our computations, probably due to precision limit of our numerical calculation.

\subsection{Raman Scattering}

The study of magnons in transition-metal ion metamagnets by light scattering has been attracting considerable experimental and theoretical interest. The well-known Raman effect is an inelastic light scattering process in which incident light of energy $\hbar \omega_{0}$ interacts with the system so that the latter is raised to some virtual intermediate states. Then relaxation occurs with simultaneous emission of light. The final stationary state can be greater, equal, or less than the initial state. The spectra of scattered light reflects the nature of internal energy system, including the magnon system of the material. The most efficient mechanism is thought to be provided by the electric dipole interaction[38]. Magnons in magnetic materials cause a spatially periodic modulation of permittivity of the medium and the light is scattered by permittivity 


\section{fluctuations.}

The small wavevector of optical radiation means that one-magnon scattering occurs in the center region of Brillouin Zone $(K=0)$, and two-magnon process arises from a pair of magnons with equal and opposite wavevectors so that again the total wavevector $(\vec{K})$ is constrained to the center of 1-st Brilliouin Zone.

The Raman Scattering in $F e B r_{2}$ has been studied by some experimental research groups, such as Psaltakis et al[25] and Johnstone et al[39]. The structure of $F e B r_{2}$ comprises layers of cations sandwiched between two layers of anions, with such composite sheets stacked long the trigonal $c$ axis. The $F e^{2+}$ layer is a two-dimensional triangular lattice. Below the Néel temperature and in zero magnetic field, the iron spins within one layer align ferromagnetically with a strong uniaxial anisotropy confining them to the trigonal axis. But adjacent layers of $\mathrm{Fe}^{2+}$ align oppositely so that the overall struture is antiferromagnetic. A general model Hamiltonian can be written as:

$$
\begin{aligned}
H= & -\sum_{i j} I_{i j}\left(S_{i}^{z} S_{j}^{z}+\sigma S_{i}^{+} S_{j}^{-}\right)-\sum_{i^{\prime} j^{\prime}} I_{i^{\prime} j^{\prime}}\left(S_{i^{\prime}}^{z} S_{j^{\prime}}^{z}+\sigma S_{i^{\prime}}^{+} S_{j^{\prime}}^{-}\right) \\
& +2 \sum_{i i^{\prime}} I_{i i^{\prime}}\left(S_{i}^{z} S_{i^{\prime}}^{z}+\gamma S_{i}^{+} S_{i^{\prime}}^{-}\right) \\
& -D\left(\sum_{i}\left(S_{i}^{z}\right)^{2}+\sum_{i^{\prime}}\left(S_{i^{\prime}}^{z}\right)^{2}\right)
\end{aligned}
$$

Here $I_{i j}$ and $I_{i^{\prime} j^{\prime}}$ are intrasublattice ferromagnetic interactions and $I_{i i^{\prime}}$ is an intersublattice coupling parameter. Since the $I_{i i^{\prime}}$ factor is usually smaller than intrasublattice one, it is interesting to compare our calculation of a pure two-dimensional triangular system with the experimental results. The basic parameters of $F e B r_{2}$ system is given 
in [25]. With the notations defined in this thesis, these parameters should correspond to

$$
\begin{gathered}
I_{1}=3.24\left(\mathrm{~cm}^{-1}\right) \\
\sigma=0.78 \\
D=2.26 I_{1}
\end{gathered}
$$

Psaltakis et al[25] identified the peak at $90.6\left(\mathrm{~cm}^{-1}\right)=27.96 I_{1}$ as a two-magnon line, and interpretated the two peaks at $29.6\left(\mathrm{~cm}^{-1}\right)=9.14 I_{1}$ and $127.5\left(\mathrm{~cm}^{-1}\right)=39.35 I_{1}$ as possibly the evidence of two-magnon bound states below and above continuum. In our calculation (Fig. 4.8 ), we found the resonance of $G(0,0)$ at $26.4 I_{1}$ and $G(1,1)$ at $33.10 I_{1}$, while lower and upper boundaries of continuum are at $9.77 I_{1}$ and $37.8 I_{1}$ respectively.

Studying the spectra shown in Fig. 4.8, and considering our discussion of the possibility of single-ion bound state at $K=0$ (section 4.1) we find that our results show good agreement with the experimental data, even though our model only consists of single layer of the triangular lattice and only nearest neighbour interactions are taken into account. Antiferromagnetic effects may have to be considered to provide interpretation of the peak above the continuum. Further study incorporating three dimensionality is certainly needed to give a more precise explanation of the experiment.

In $\mathrm{FeBr} r_{2}$ the ratio of the NN interlayer coupling parameter to the NN intralayer parameter is 0.29 . The same ratio in $\mathrm{FeCl}_{2}$, which has similiar magnetic structure, 
is only 0.05 [40]. In this sense $\mathrm{FeCl}_{2}$ seems even closer to our 2-D triangular model.

However the two-magnon scattering peak in $\mathrm{FeCl}_{2}$ was not discovered in [40]. 


\section{Chapter 5}

\section{Conclusion}

The behaviour of two-magnon bound states and resonances of the 2D NN triangular Heisenberg ferromagnet was examined for all total pair wave vectors $\vec{K}$ in three high symmetry directions. The general results are found consistent with the previous work in the 3-D and the plane square lattices. However the more complicated nature of triangular lattice does give rise of some interesting phenomena which were not present in simpler lattice structures. The effect of spin magnitude $S$, which is rarely considered by other researchers, is found to be quite significant to the formation of bound states. While the number of bound states only depends on the dimension of lattice in simple lattices [27], this is not the case in triangular lattice. Instead its formation and evolution is strongly influenced by all parameters, $S, D$, and $\sigma$. The triangular case shares with the NN FCC case a non-zero minimum width of the continuum(as plotted as a function of $\vec{K}$ ). It also has a much complicated internal structure of the non-interacting spectrum as shown by our study of the diagrams of its critical lines. For large enough $S$ the bound states may not form outside the 
continuum, which is unlike the hypercubic cases. A strong enough $D$ always produces a single-ion bound state, while a small enough $\sigma$ helps eject bound states.

At the zone center $(K=0)$, the resonance of $G(0,0)$ is relatively not affected by $D$-anisotropy even when $D$ produces a gap below the continuum and lifts the whole band up, but it is shifted correspondingly when $\sigma$-anisotropy squeezes the band. On the other hand, the $G(1,1)$ resonance is moving closely following the shift and deformation of the band, but the position of the resonance with respect to the band remains relatively unchanged. The evolution of the continuum as a function of $\vec{K}$ also becomes more complicated. The critical lines still play important roles in determination of resonance frequencies. The general trends elucidated give a reasonable degree of confidence that the simpler analysis of unperturbed continuum structure would enable one to predict the behaviour of the resonances once a starting point has been established.

The Green's function method can be extended to a more complete analysis of the processes in Raman scattering experiments. The present work can also be extended to some other directions, such as the case of next nearest neighbour interaction (NNN) and the case of complex lattices where the inversion symmetry is broken, say, the diatomic chain, etc. 


\section{Appendix $\mathbf{A}$}

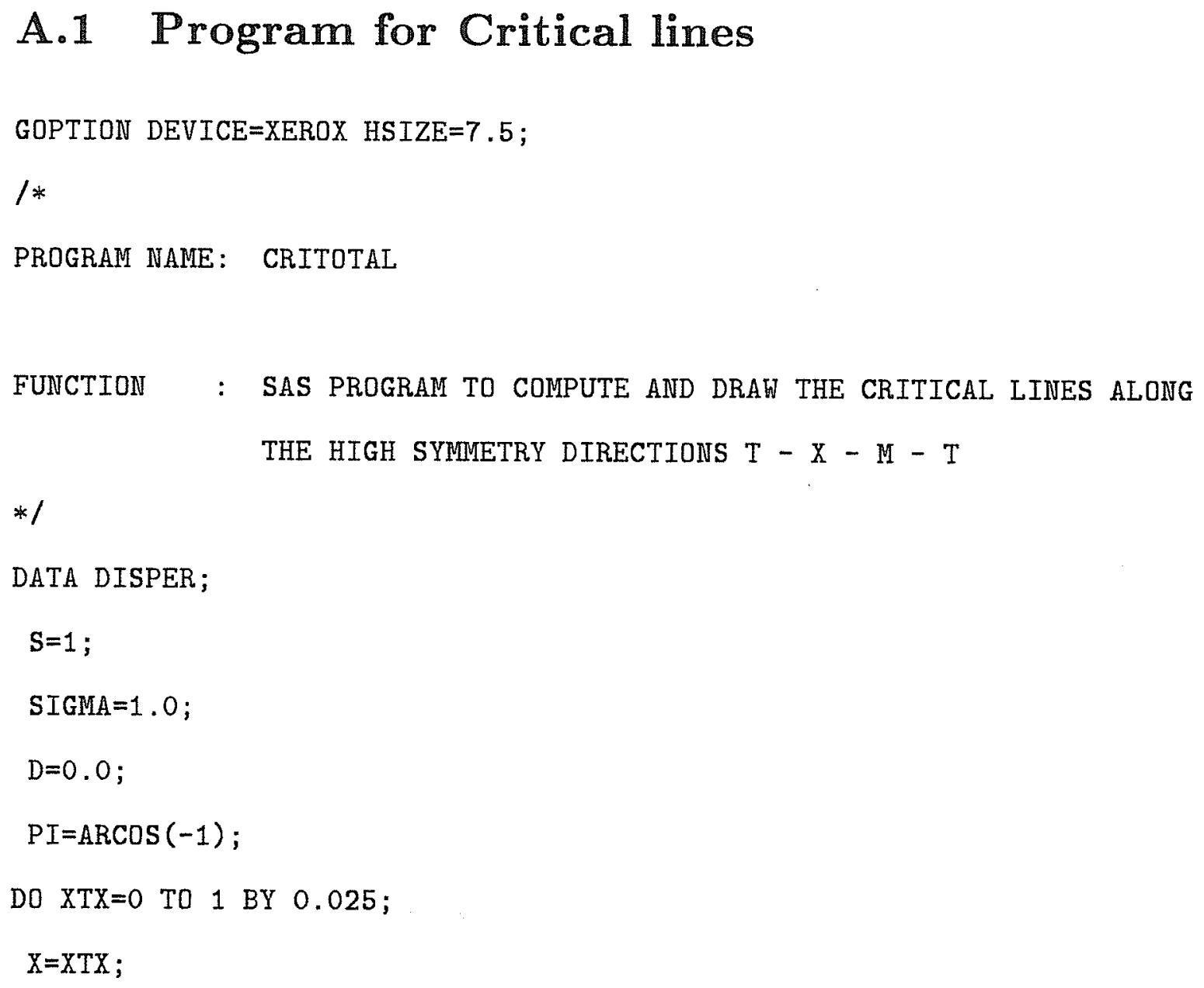




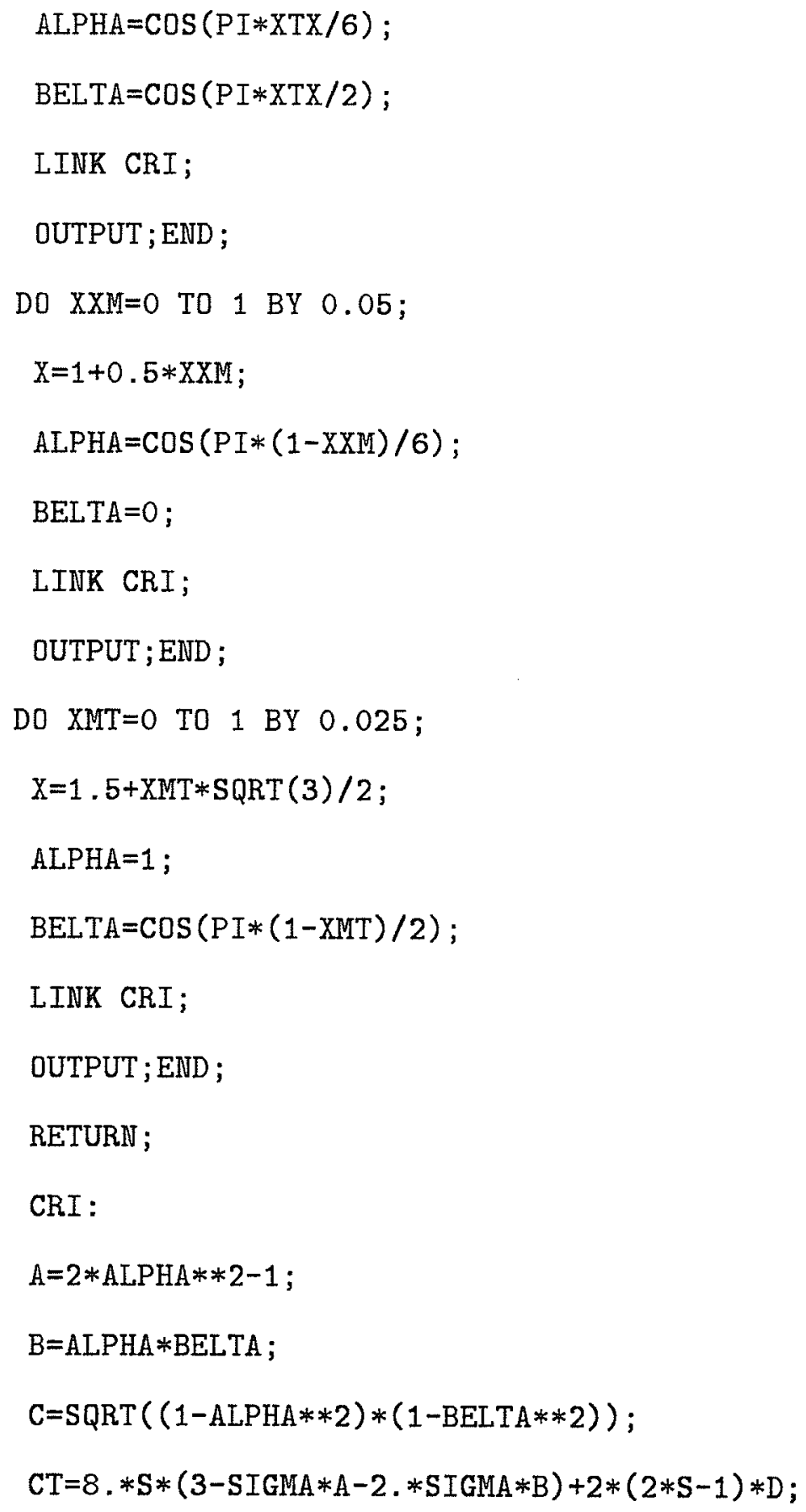




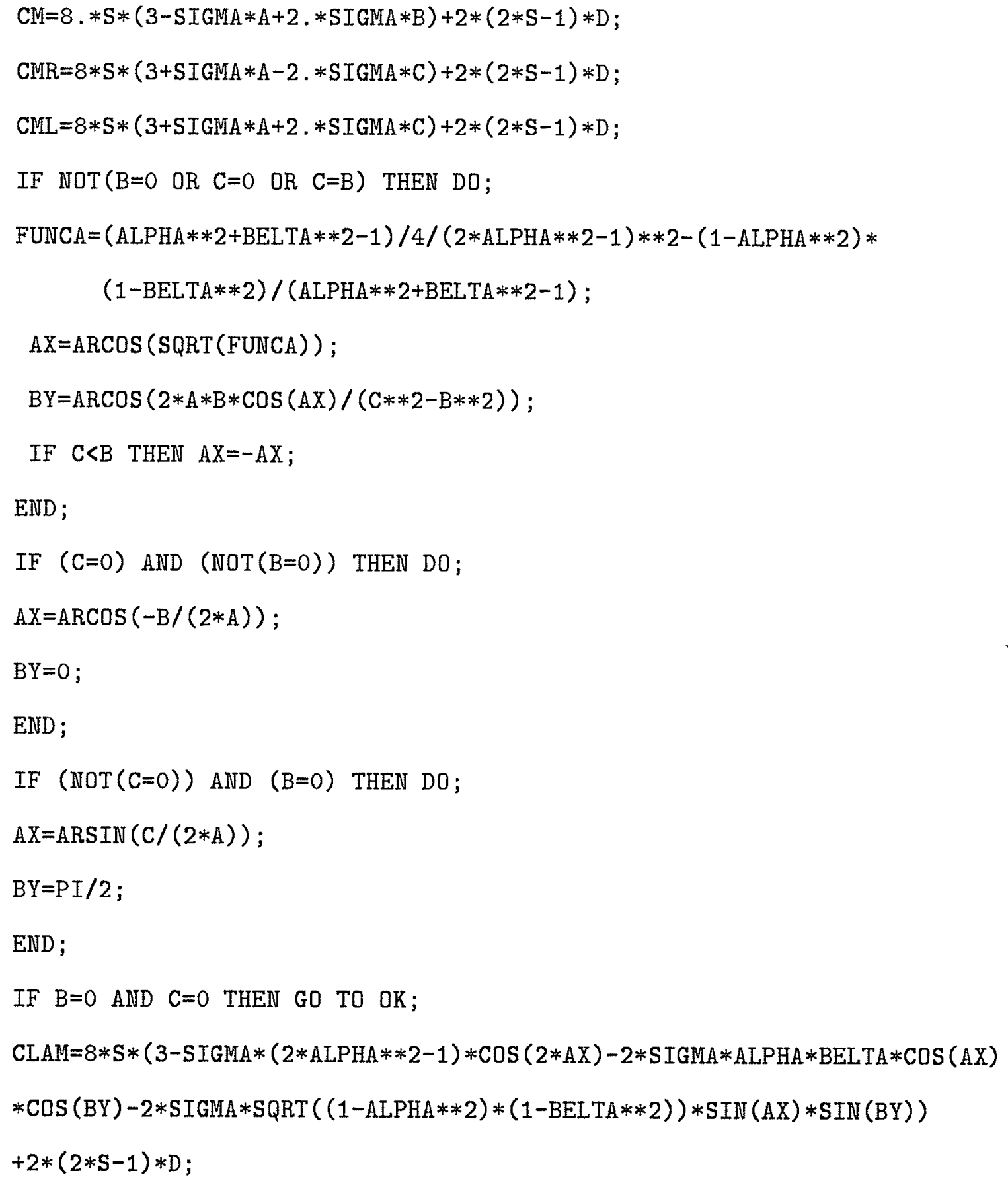


OK : RETURN;

PROC GPLOT;

PLOT (CT CM CMR CML CLAM) $* \mathrm{X}=1$

\section{/OVERLAY}

FRAME

SKIPMISS

$\mathrm{HREF}=1.0 \quad 1.5 \quad 2.37$

VAXIS $=A X I S 2$

HAXIS=AXIS1;

PLOT2 (CT CM CMR CML CLAM) $* \mathrm{X}=1$

/OVERLAY
FRAME
SKIPMISS
HREF=1.0 $1.5 \quad 2.37$
VAXIS=AXIS 2
HAXIS=AXIS1;

AXIS1 ORDER $=0.0$ TO 2.37 BY 0.01

LENGTH $=11.25 \mathrm{CM}$

LABEL $=$ NONE

VALUE $=$ NONE

MAJOR $=$ NONE

MINOR= NONE; 


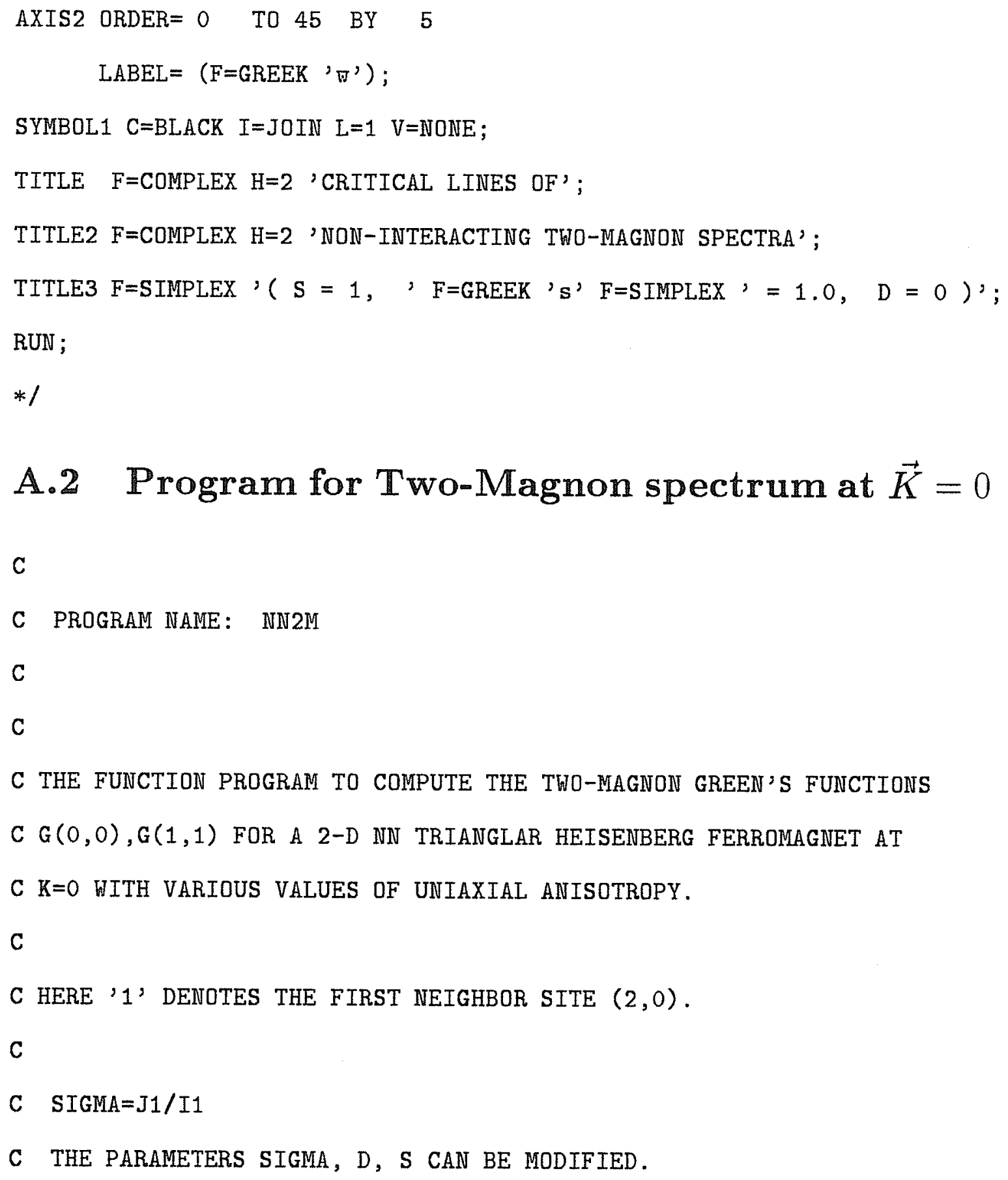




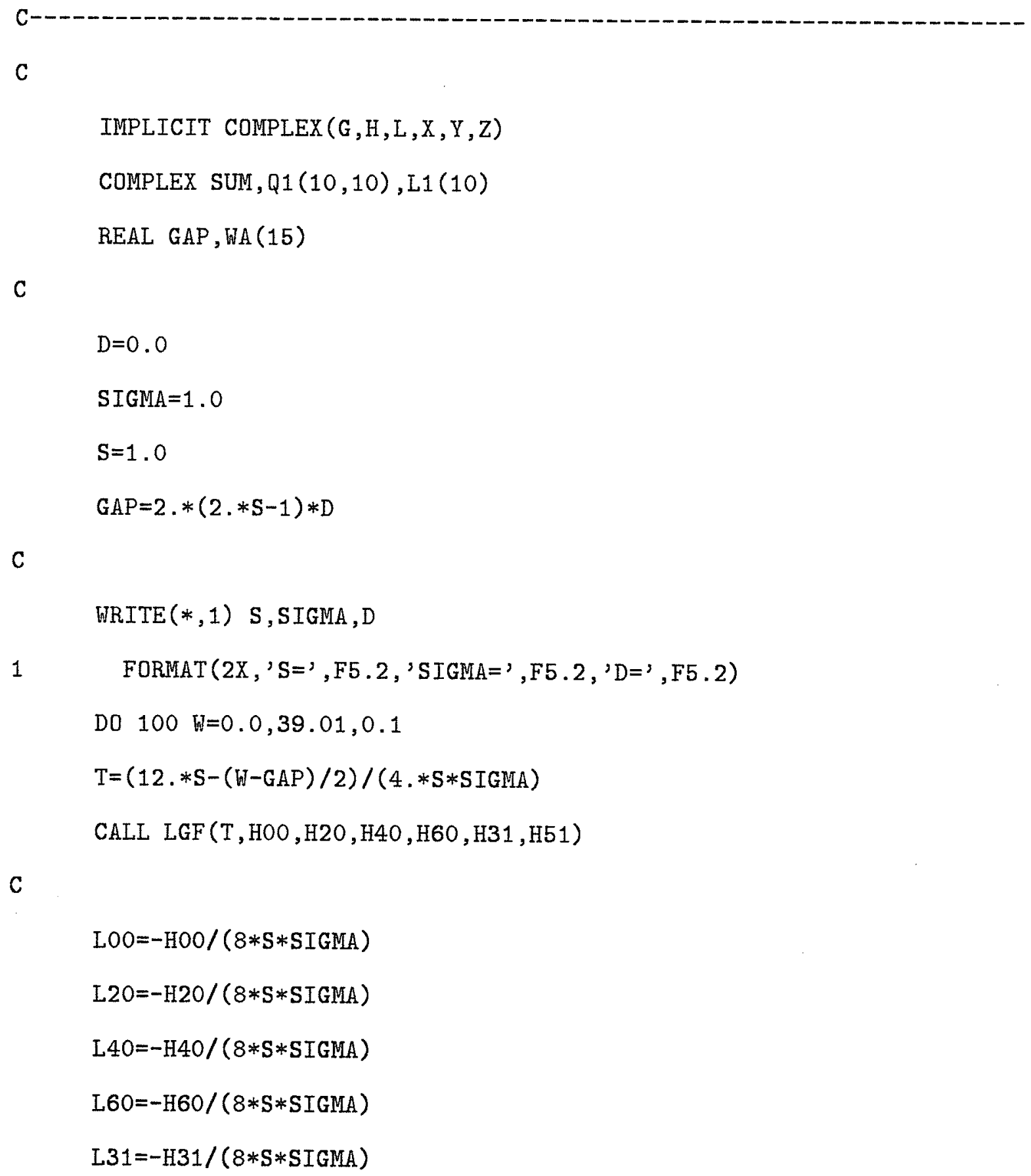




$$
\mathrm{L} 51=-\mathrm{H} 51 /(8 * \mathrm{~S} * \mathrm{SIGMA})
$$

C

C Calculate $\mathrm{G}(0,0)$

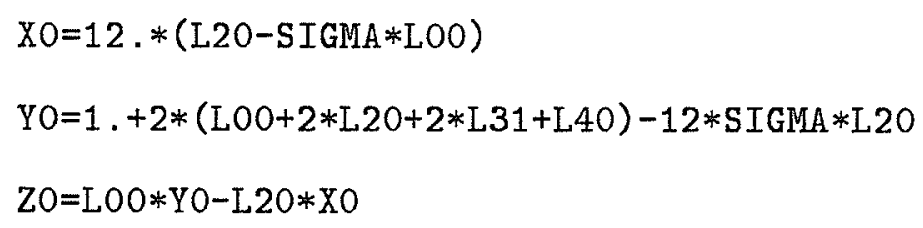

C

$$
\mathrm{GOO}=4 * \mathrm{~S} * * 2 *(2 .-1.0 / \mathrm{S}) * \mathrm{ZO} /(\mathrm{YO}+2 * \mathrm{D} * \mathrm{ZO})
$$

C

C CALCULATE G(1,1)

C

$$
\begin{aligned}
& \mathrm{X} 1=2 *(\mathrm{~L} 20+\mathrm{L} 31)-4 * \mathrm{SIGMA} * \mathrm{~L} 20 \\
& \mathrm{Y} 1=1 .+2 *(\mathrm{~L} 00+\mathrm{L} 40)-4 * \mathrm{SIGMA} * \mathrm{~L} 20 \\
& \mathrm{Z} 1=4 * \mathrm{~L} 20-4 * \mathrm{SIGMA} * \mathrm{~L} 00
\end{aligned}
$$

C

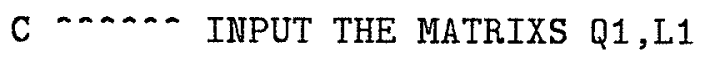

DO $10 I=1,3$

DO $5 \quad I 1=1,3$

5

$$
\begin{aligned}
& \mathrm{Q} 1(I, I 1)=\mathrm{X} 1 \\
& \mathrm{Q} 1(I, I)=\mathrm{Y} 1 \\
& \mathrm{Q} 1(I, 4)=2 . * \mathrm{D} * \mathrm{I} 20
\end{aligned}
$$




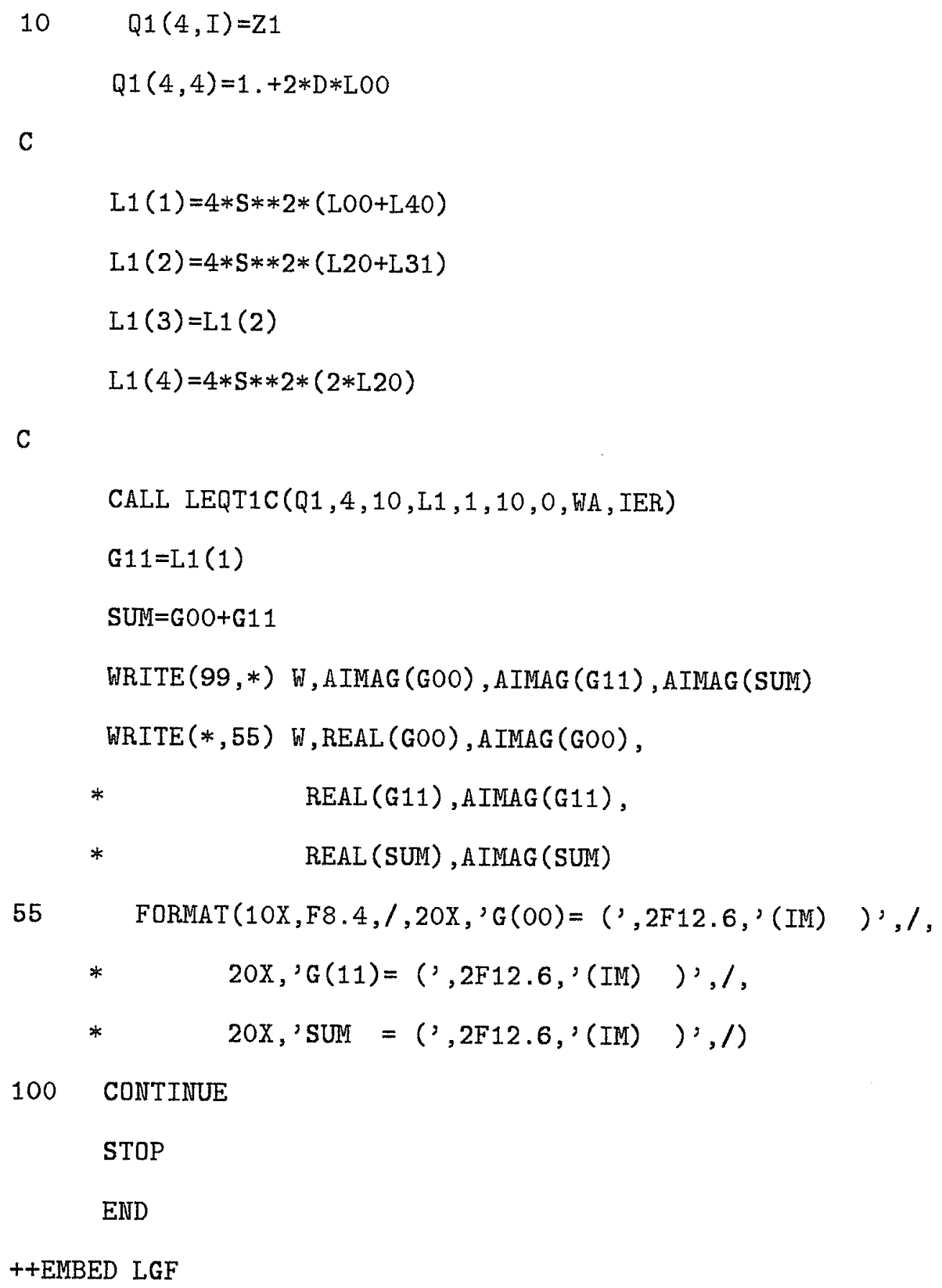


++EMBED ELLIP

$/ /$ GD.FT99F001 DD DSN=XQU. GREENOOO.DATA, DISP $=S H R$

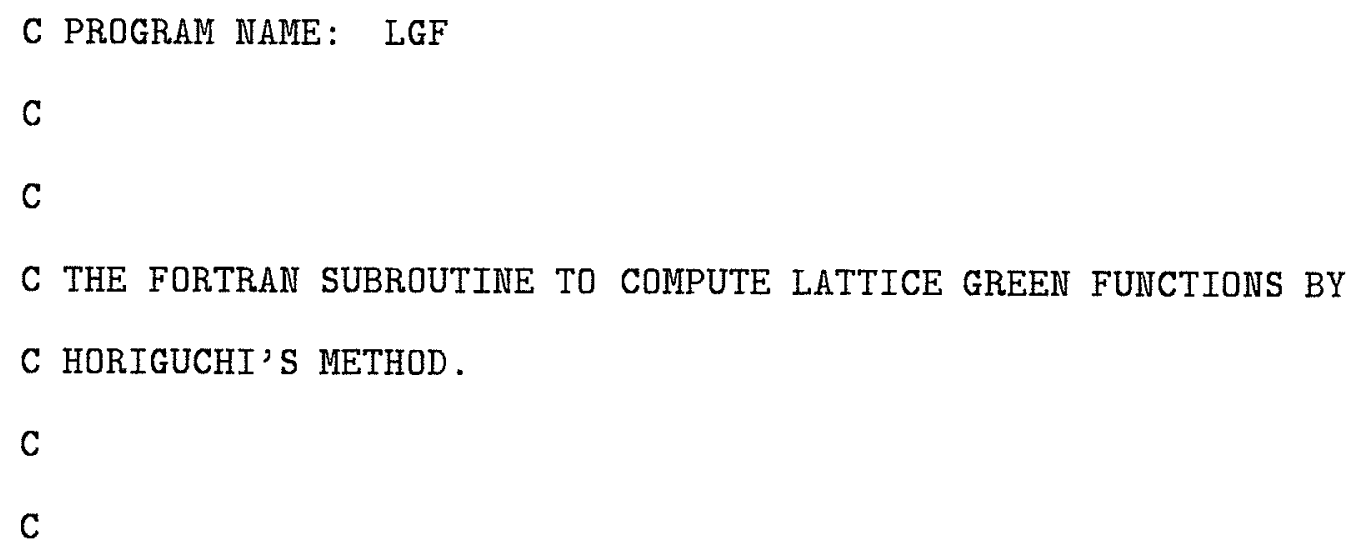

CALL ELLIP $(\mathrm{KV}, \mathrm{KF}, \mathrm{EF}, \mathbb{N})$ 
C

IF (N.EQ.O) THEN

$\operatorname{WRITE}(*, 20) \mathrm{S}, \mathrm{KV}$

20 FORMAT (2X, 'S=',F5.2,' $\mathrm{KV}=\left({ }^{\prime}, 2 \mathrm{~F} 10.6,{ }^{\prime}\right)$--.-SINGULARITY

C POINT OF ELLIPTIC INTEGRAL----')

GOTO 100

END IF

C

IF (AIMAG (KV).GT.0.0) THEN

$\mathrm{KBAR}=\mathrm{KF}$

$\mathrm{EBAR}=\mathrm{EF}$

ELSE

CALL ELLIP (SQRT (1-KV**2), KFC,EFC,N)

$\mathrm{KBAR}=\mathrm{KF}-(0.0,2.0) * \mathrm{KFC}$

$\mathrm{EBAR}=\mathrm{EF}-(0.0,2.0) *(\mathrm{KFC}-\mathrm{EFC})$

END IF

C

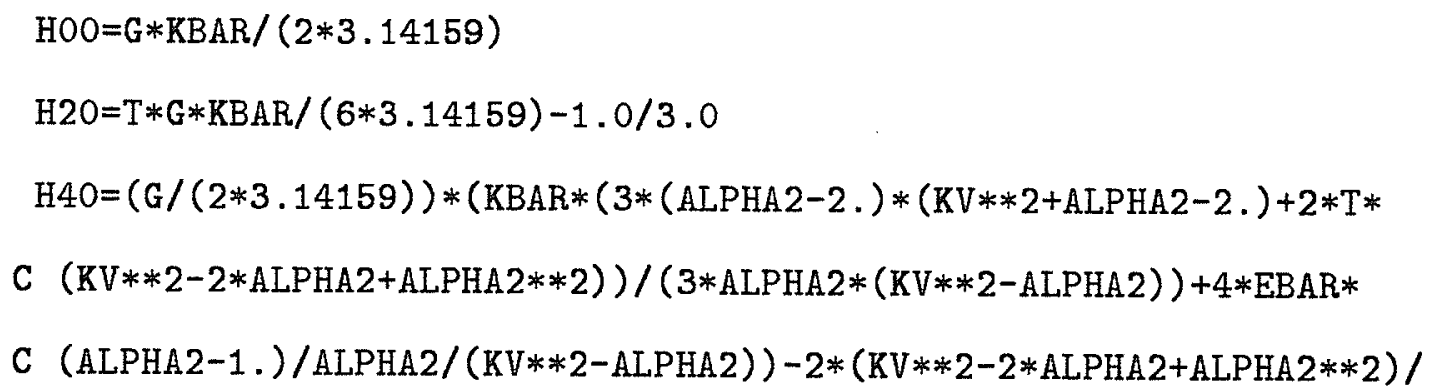


C $(3 *$ ALPHA $2 *(K V * * 2-A L P H A 2))$

C

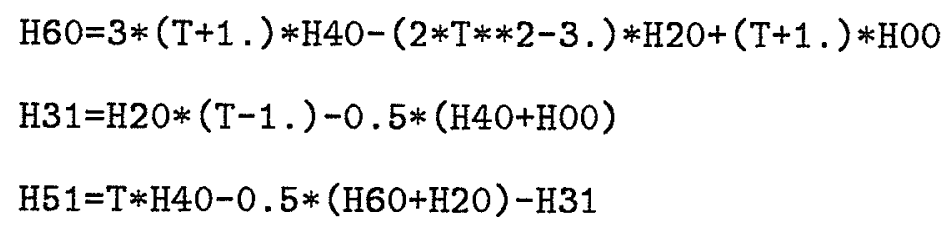

C

100 RETURN

END

C PROGRAM NNAME: ELLIP

C

C THE FORTRAN SUBROUTINE TO EVALUATE THE COMPLETE ELLIPTIC INTEGRALS

C WITH A COMPLEX MODULUS.

C

C

C

SUBROUTINE ELLIP $(K V, K F, E F, N)$

IMPLICIT COMPLEX (K)

COMPLEX A, C, S, EF

C

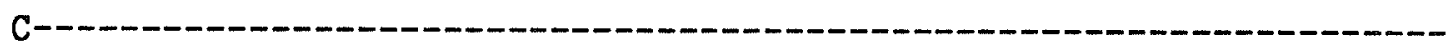

C 


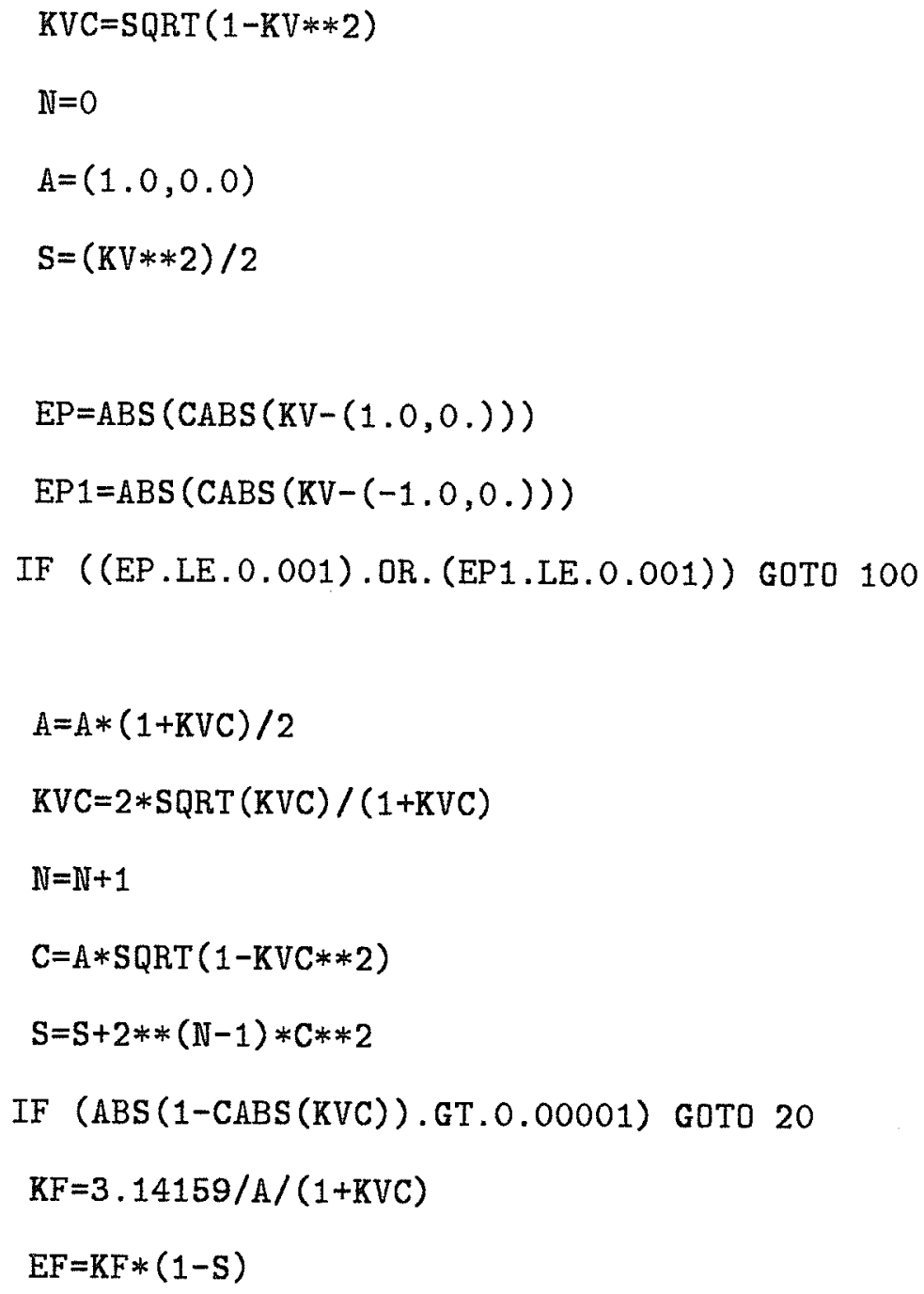

END 
GOPTION DEVICE=XEROX HSIZE=7.5;

/*

PROGRAM NAME: DRAWNN

FUNCTION : THE SAS PROGRAM TO DRAW THE SPECTRA OF TWO-MAGNON GREEN FUNCTION, TAKING DATA FROM THE RESULTS OF RUNNING 'NN2M' OR 'GREEN' .

*/

DATA NN2M;

INFILE MAGNON;

* INPUT $X$ V REG00 IMG00 REG11 IMG11 REG112 IMG112 REG113 IMG113;

INPUT 6 IMGOO IMG11 IMG112;

IMGOO=-IMGOO;

IMG11=-IMG11;

IMG112=-IMG112;

IMG113=-IMG113;

IF IMGOO >2.49 THEN IMGOO $=2.49$;

IF IMG11 >2.49 THEN IMG11 $=2.49$;

IF IMG112>2.49 THEN IMG112=2.49;

PROC SORT;

BY W;

SYMBOL1 C=BLACK V=STAR I=JOIN; 
SYMBOL2 C=BLACK I=JOIN;

SYMBOL3 $C=B L A C K \quad L=20$ I=JOIN;

PROC GPLOT;

PLOT IMG00*W=1 IMG11*W=2 IMG112*W=3/OVERLAY

$\mathrm{VREF}=0$

HAXIS=AXIS2

VAXIS=AXIS1;

AXIS1 ORDER $=0.0$ TO 2.5 BY 0.1

LABEL = NONE;

AXIS2 ORDER $=0.0$ TO 50.0 BY 10.

LABEL = (F=GREEK ' $\nabla$ ' $)$;

TITLE F=COMPLEX 'TWO MAGNON PROPAGATORS IN 2-D TRIANGULAR LATTICE';

TITLE2 $\mathrm{F}=\mathrm{SIMPLEX}$ ' $(\mathrm{SIGMA}=\mathrm{J} 1 / \mathrm{I} 1=1.0, \mathrm{~S}=1, \mathrm{D}=0.0, \mathrm{~K}=0.0$ along' $F=S I M P L E X$ ' $T-X)$ ';

FOOTNOTE F=SIMPLEX 'STAR LINE --- $-\operatorname{Im}(\mathrm{G}(0,0) \quad)$;

FOOTNOTE2 F=SIMPLEX 'SOLID LINE --- $-\operatorname{Im}(\mathrm{G}(1,1)$ )';

FOOTNOTE3 F=SIMPLEX 'DASHED LINE --- $-\operatorname{Im}\left(\mathrm{G}\left(1^{6}, 1^{6}\right)\right)^{\prime}$;

RUN;

\section{A.3 Program for Two-Magnon Spectrum at ger- enal $\vec{K}^{\prime}$ s}

C PROGRAM NAME: GREEN 
C THE FORTRAN PROGRAM TO COMPUTE THE SPECTRA OF TWO-MAGNON GREEN'S

C FUNCTIONS $\mathrm{G}(0,0), \mathrm{G}(1,1)$ AND $\mathrm{G}\left(1^{\prime}, 1^{\prime}\right)$, BOTH CONTINUUM AND BOUND

C STATES, OF 2-D NN TRIANGLAR HEISENBERG FERROMAGNET WITH VARIOUS

C VALUES OF ANISOTROPY WHILE THE TOTAL WAVEVECTOR $K$ MOVES ALONG

C THE HIGH SYMMETRY LINES IN $1 \mathrm{ST}$ BZ.

C

C HERE "1" AND " 1 ' " DENOTE THE FIRST NEIGHBor SITE $(2,0)$ AND

C $(1,1)$ RESPECTIVELY.

C

C $\operatorname{SIGMA}=\mathrm{J} 1 / \mathrm{I} 1$

C IN THE PROGRAM SIGMA, D, S, CAN BE MODIFIED

C

IMPLICIT COMPLEX ( $L, R, F, G)$

REAL GAP, IMLGF (10), RELGF (10)

$\mathrm{REAL} * 8 \mathrm{~T}, \mathrm{~A}, \mathrm{~B}, \mathrm{C}$

COMPLEX $Q(10,10), Q H O L D(10,10), R(10)$, WA (15)

INTEGER L

COMMON /T/T , /ABC/A,B, C,/L/IMLGF , RELGF

C 


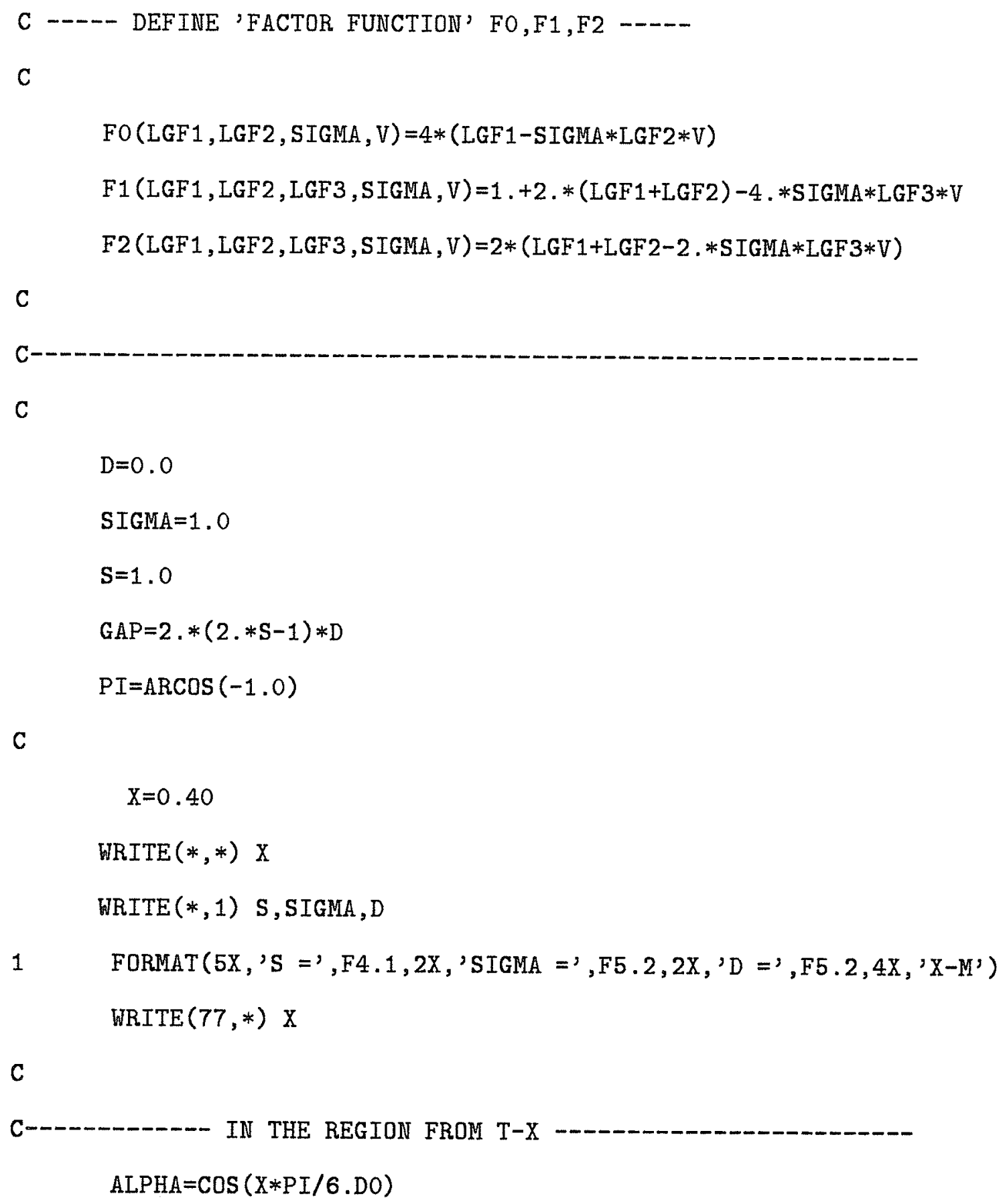


$\mathrm{BETA}=\operatorname{COS}(\mathrm{X} * \mathrm{PI} / 2 . \mathrm{DO})$

C

C-1N THE REGION FROM X-M -

C $\quad$ ALPHA $=\operatorname{COS}((1 .-\mathrm{X}) * \mathrm{PI} / 6 . \mathrm{DO})$

C BETA $=0$.

C

C-D IN THE REGION FROM X-T -

C ALPHA $=1.0$

C $\quad B E T A=\operatorname{COS}((1,-X) * P I / 2$.

C

C-1

C

$A=2 . D O * A L P H A * * 2-1 . D O$

$B=A L P H A * B E T A$

$C=\operatorname{SQRT}((1-\operatorname{ALPHA} * * 2) *(1-\operatorname{BETA} * * 2))$

DO $220 \quad W=12.941,12.9441,0.001$

$\mathrm{T}=(12 . * \mathrm{~S}-(\mathrm{W}-\mathrm{GAP}) / 2 . \mathrm{DO}) /(4 . * \mathrm{~S} * \mathrm{SIGMA})$

CALL LGF

C

$$
\begin{aligned}
& \text { LO0 }=-\operatorname{CMPLX}(\text { RELGF }(1), \operatorname{IMLGF}(1)) /(8 * \mathrm{~S} * \mathrm{SIGMA}) \\
& \text { L20 }=-\operatorname{CMPLX}(\operatorname{RELGF}(2), \operatorname{IMLGF}(2)) /(8 * \mathrm{~S} * \mathrm{SIGMA}) \\
& \operatorname{L} 40=-\operatorname{CMPLX}(\operatorname{RELGF}(3), \operatorname{IMLGF}(3)) /(8 * \mathrm{~S} * \mathrm{SIGMA})
\end{aligned}
$$




$$
\begin{aligned}
& Q(2,4)=F 2(L 11, \text { LN31, L20, SIGMA , B+C }) \\
& Q(4,2)=F 2(L 11, \text { LN31, LN11, SIGMA , A ) } \\
& Q(3,4)=F 2(L 20, \text { L02, L11, SIGMA , B+C) } \\
& Q(4,3)=F 2(L 20, \text { L02, LN11, SIGMA , B-C) }
\end{aligned}
$$

C

DO $20 \quad I 1=1,4$

DO $20 \quad I 2=1,4$

$20 \quad \mathrm{QHOLD}(I 1, I 2)=\mathrm{Q}(\mathrm{I} 1, \mathrm{I} 2)$

C

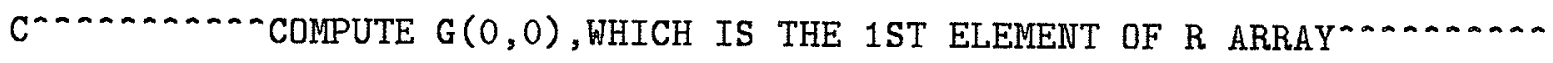
C

$$
\begin{aligned}
& R(1)=(4 * S * * 2) *(2-1 / S) * \mathrm{~L} 00 \\
& R(2)=(4 * S * * 2) *(2-1 / S) * \mathrm{~L} 20 \\
& R(3)=(4 * S * * 2) *(2-1 / S) * \mathrm{~L} 11 \\
& R(4)=(4 * S * * 2) *(2-1 / S) * \operatorname{LN} 11
\end{aligned}
$$

C

CALL LEQT1C $(Q, 4,10, R, 1,10,0, W A$, IER $)$

C

$$
\mathrm{GOO}=\mathrm{R}(1)
$$

C

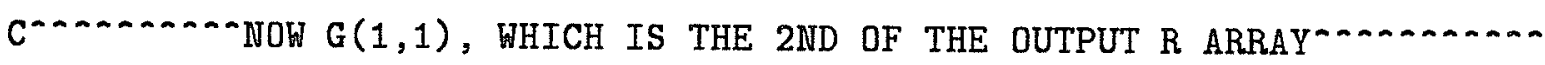
$R(1)=4 * S * 2 *(2 * L 20)$ 


$$
\begin{aligned}
& R(2)=4 * S * 2 *(L 00+L 40) \\
& R(3)=4 * S * 2 *(L N 11+L 31) \\
& R(4)=4 * S * 2 *(L 11+L N 31)
\end{aligned}
$$

C

DO $30 \quad I 1=1,4$

DO $30 \quad I 2=1,4$

$30 \quad \mathrm{Q}(\mathrm{I} 1, \mathrm{I} 2)=\mathrm{QHOLD}(\mathrm{I} 1, \mathrm{I} 2)$

C

CALL LEQT1C $(Q, 4,10, R, 1,10,0$, WA, IER $)$

C

$\mathrm{G} 11=\mathrm{R}(2)$

C

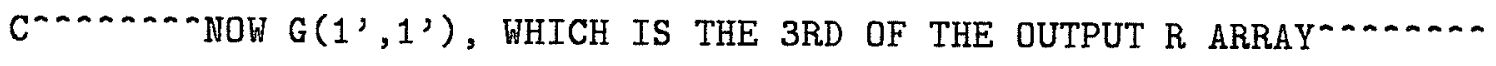

$R(1)=4 * S * * 2 *(2 * I 11)$

$R(2)=4 * S * 2 *(\operatorname{LN} 11+\mathrm{L} 31)$

$R(3)=4 * S * 2 *(L 00+L 22)$

$R(4)=4 * S * * 2 *(\mathrm{~L} .02+\mathrm{L} 20)$

C

DO $40 \quad I 1=1,4$

DO $40 \quad I 2=1,4$

40

$Q(I 1, I 2)=Q H O L D(I 1, I 2)$

C 
CALI LEQT1C $(Q, 4,10, R, 1,10,0, \mathrm{HA}$, IER $)$

C

$\mathrm{G} 112=\mathrm{R}(3)$

C

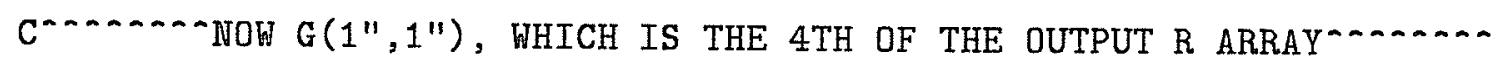

$R(1)=4 * S * 2 *(2 * \operatorname{LN} 11)$

$R(2)=4 * S * * 2 *(L 11+\operatorname{LN} 31)$

$R(3)=4 * S * * 2 *(\mathrm{~L}, 02+\mathrm{L} 20)$

$R(4)=4 * S * * 2 *(L 00+\operatorname{LN} 22)$

C

DO $50 \quad I 1=1,4$

DO $50 \quad I 2=1,4$

$50 \quad \mathrm{Q}(\mathrm{I} 1, \mathrm{I} 2)=\mathrm{QHOLD}(\mathrm{I} 1, \mathrm{I} 2)$

C

CALI LEQT1C $(Q, 4,10, R, 1,10,0$, WA, IER $)$

C

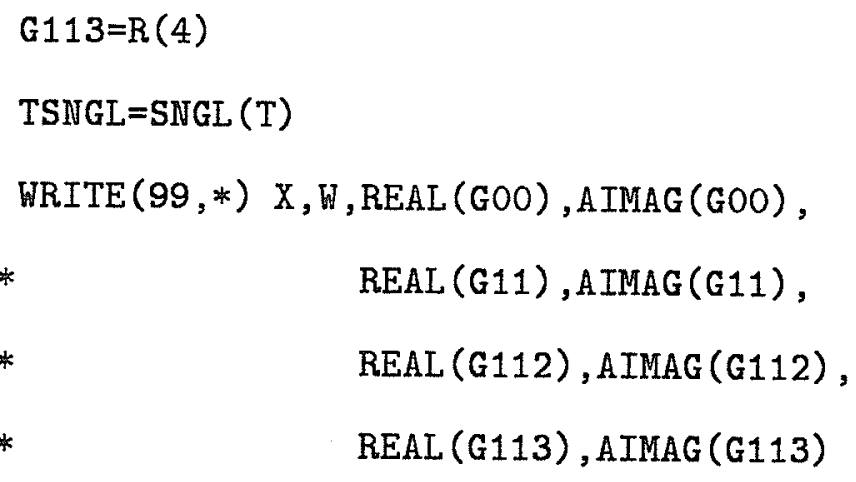




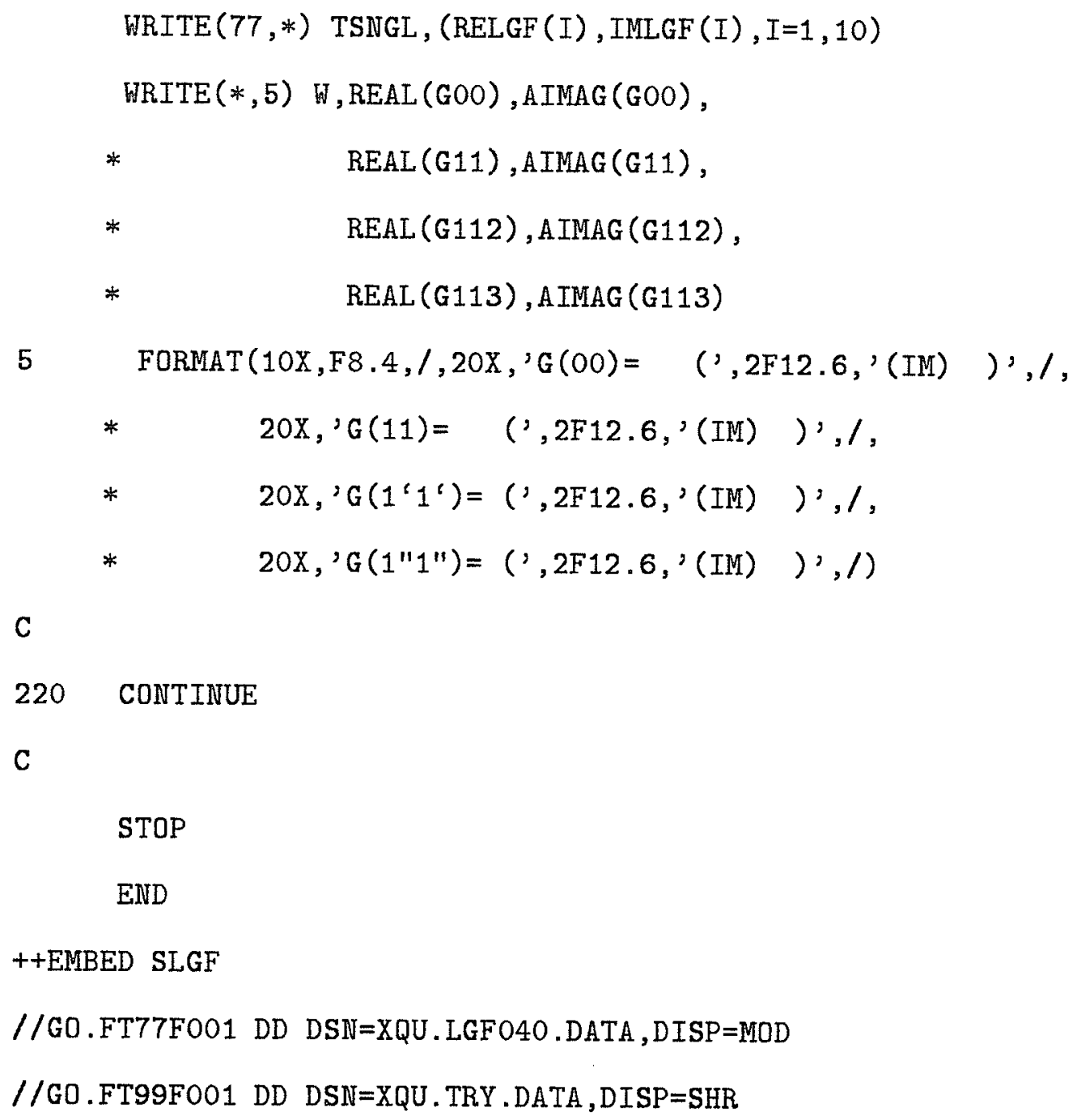

C PROGRAM NAME: LGF

C 
C

C THE FORTRAN PROGRAM CALCULATE THE REAL AND IMAGINARY PARTS

C OF THE LATTICE GREEN FUNCTION $G(I, J ; E)$ IN 2-D TRIANGULAR

C LATTICE WITH TWO-CORNER DEGENERACY

C

C VARIABLE LIST

C

C APREG - THE APPROXIMATE VALUE OF THE REAL PART OF G(T)

C APIMG - THE APPROXIMATE VALUE OF THE IMAGINARY PART OF G(T)

C EK - AN N BY N ARRAY HOLDING THE EIGENVALUES

C FK - AN N BY N ARRAY HOLDING THE MATRIX ELEMENTS

C

C THE REAL AND IMAGINARY PARTS OF THE LATTICE GREEN FUNCTIONS

C CALCULATED ARE RESTORED IN 2 ARRAYS 'RELGF(10)' \& 'IMLGF(10)' IN

C THE ORDER SPECIFIED BY 'XINDEX' AND 'YINDEX'. THE CURRENT ORDER IS

C $\quad(00) ;(20) ;(40) ;(-31) ;(-11) ;(11) ;(31) ;(-22) ;(02) ;(22)$

C

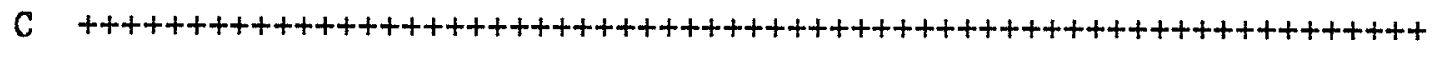

C

SUBROUTINE LGF

IMPLICIT REAL*8 $(\mathrm{A}-\mathrm{H}, \mathrm{O}-\mathrm{Z})$

DIMENSION EK $(-200: 200,0: 200), F K(-200: 200,0: 200), \operatorname{EN}(3), F(3)$ 
REAL*4 IMLGF (10), RELGF (10)

C

COMMON EK, FK

COMMON /T/T , /PI/PI , /ENF/EN , F, /AP/APIMG , APREG

COMMON /L/IMLGF, RELGF

$\mathrm{PI}=\mathrm{DARCOS}(-1 . \mathrm{DO})$

C

$N=199$

$\mathrm{R} 8 \mathrm{~N}=199$. DO

C

DO $20 \mathrm{~L}=1,10$

$\operatorname{RELGF}(\mathrm{L})=0$.

$\operatorname{IMLGF}(L)=0$.

CALL GRID $(\mathbb{N}, R 8 N, L)$

DO $20 \mathrm{M} 2=0, \mathrm{~N}-1,1$

DO $20 \mathrm{M} 1=-\mathrm{N}, \mathrm{N}-1,1$

C

$$
\begin{aligned}
& \mathrm{M} 3=\mathrm{M} 1+1 \\
& \mathrm{M} 4=\mathrm{M} 2+1
\end{aligned}
$$

c

$$
\begin{aligned}
& \operatorname{EN}(1)=\operatorname{EK}(M 1, M 2) \\
& \operatorname{EN}(2)=\operatorname{EK}(M 1, M 4)
\end{aligned}
$$




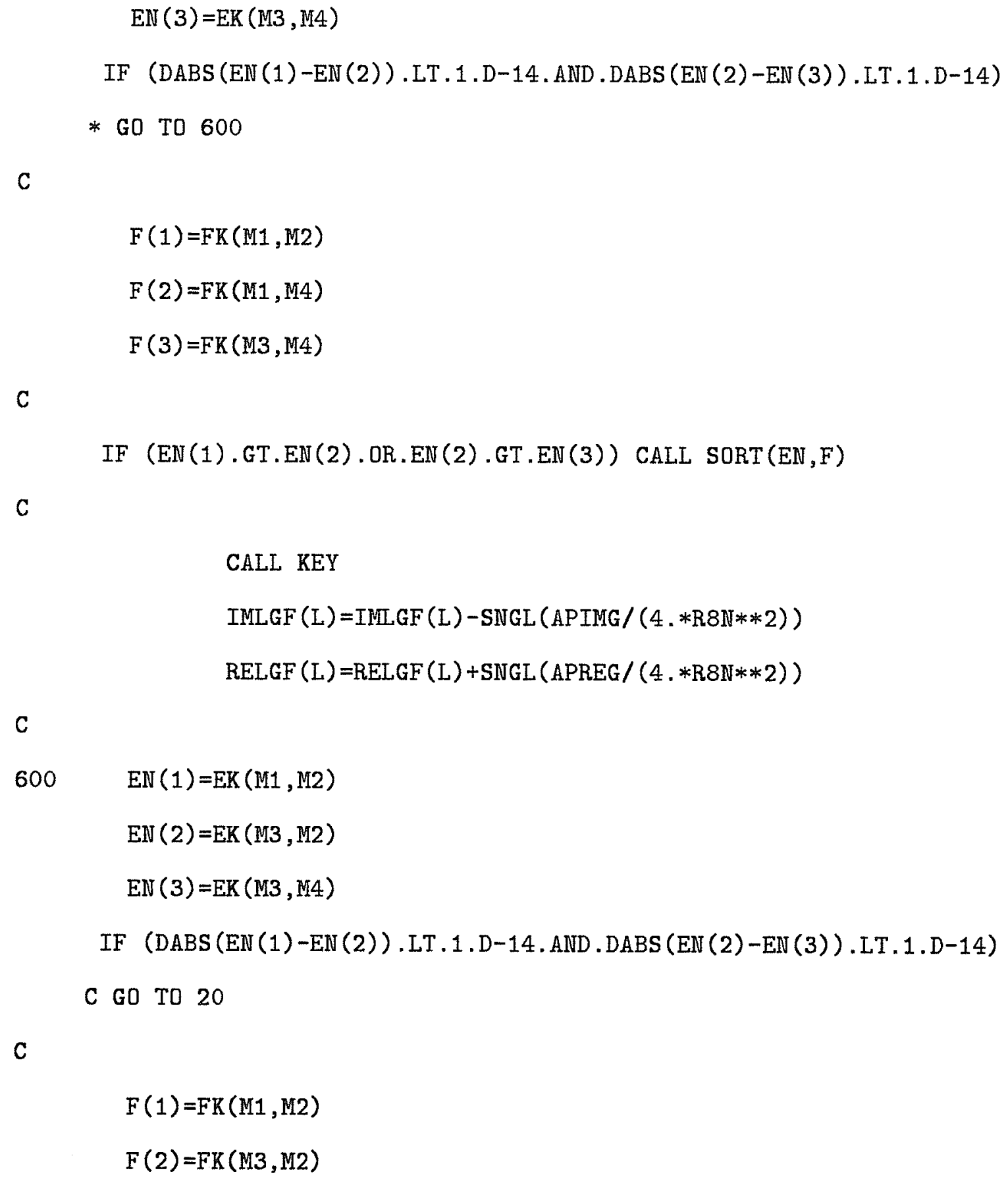

$$
\begin{aligned}
& F(1)=F K(M 1, M 2) \\
& F(2)=F K(M 3, M 2)
\end{aligned}
$$




$$
F(3)=F K(M 3, M 4)
$$

C

IF (EN(1).GT.EN(2).OR.EN(2).GT.EN(3)) CALL SORT(EN,F)

C

CALL KEY

$\operatorname{IMLGF}(\mathrm{L})=\operatorname{IMLGF}(\mathrm{L})-\operatorname{SNGL}(\operatorname{APIMG} /(4 . * \mathrm{R} 8 \mathrm{~N} * * 2))$

$\operatorname{RELGF}(\mathrm{L})=\operatorname{RELGF}(\mathrm{L})+\operatorname{SNGL}(\operatorname{APREG} /(4 . * \mathrm{R} 8 \mathrm{~N} * * 2))$

20 CONTINUE

C

RETURN

END

C

$\mathrm{C} * * * * * * * * * * * * * * * * * * * * * * * * * * * * * * * * * * * * * * * * * * * * * * * * * * * * * * * * * * * * * * * *$

$\mathrm{C}$

SUBRDUTINE GRID (N, R8N, L)

$\mathrm{C}$

C THIS SUBROUTINE CALCULATES THE ENERGY EIGENVALUES AND

C AND MATRIX ELEMENTS

C

IMPLICIT REAL $* 8(\mathrm{~A}-\mathrm{H}, \mathrm{O}-\mathrm{Z})$

DIMENSION EK $(-200: 200,0: 200), F K(-200: 200,0: 200), X I N D E X(10)$, *YINDEX (10) 
C

RETURN

END

C

C $* * * * * * * * * * * * * * * * * * * * * * * * * * * * * * * * * * * * * * * * * * * * * * * * * * * * * * * * * * * * * * *$

C

SUBROUTINE KEY

C

C THIS SUBROUTINE CALCULATES THE CONTRIBUTION OF A TRIANGULAR CELL

C

IMPLICIT REAL*8 $(\mathrm{A}-\mathrm{H}, \mathrm{O}-\mathrm{Z})$

DIMENSION EN (3),F(3)

COMMON /T/T /PI/PI /ENF/EN, F / AP/APIMG, APREG

$A P I M G=0 . D O$

$A P R E G=0 . D O$

C

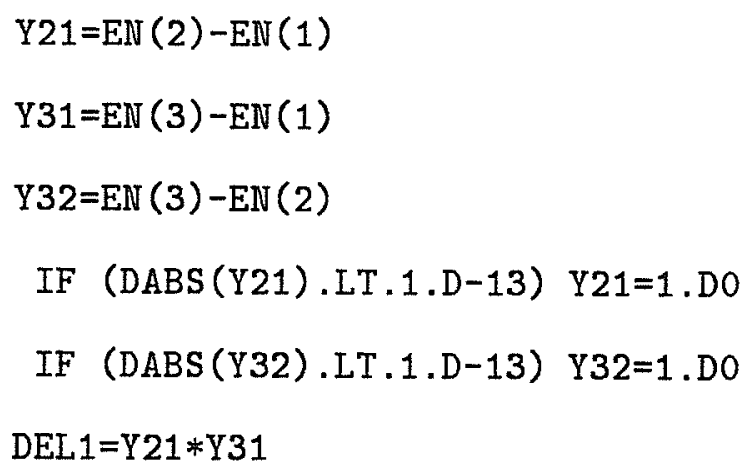




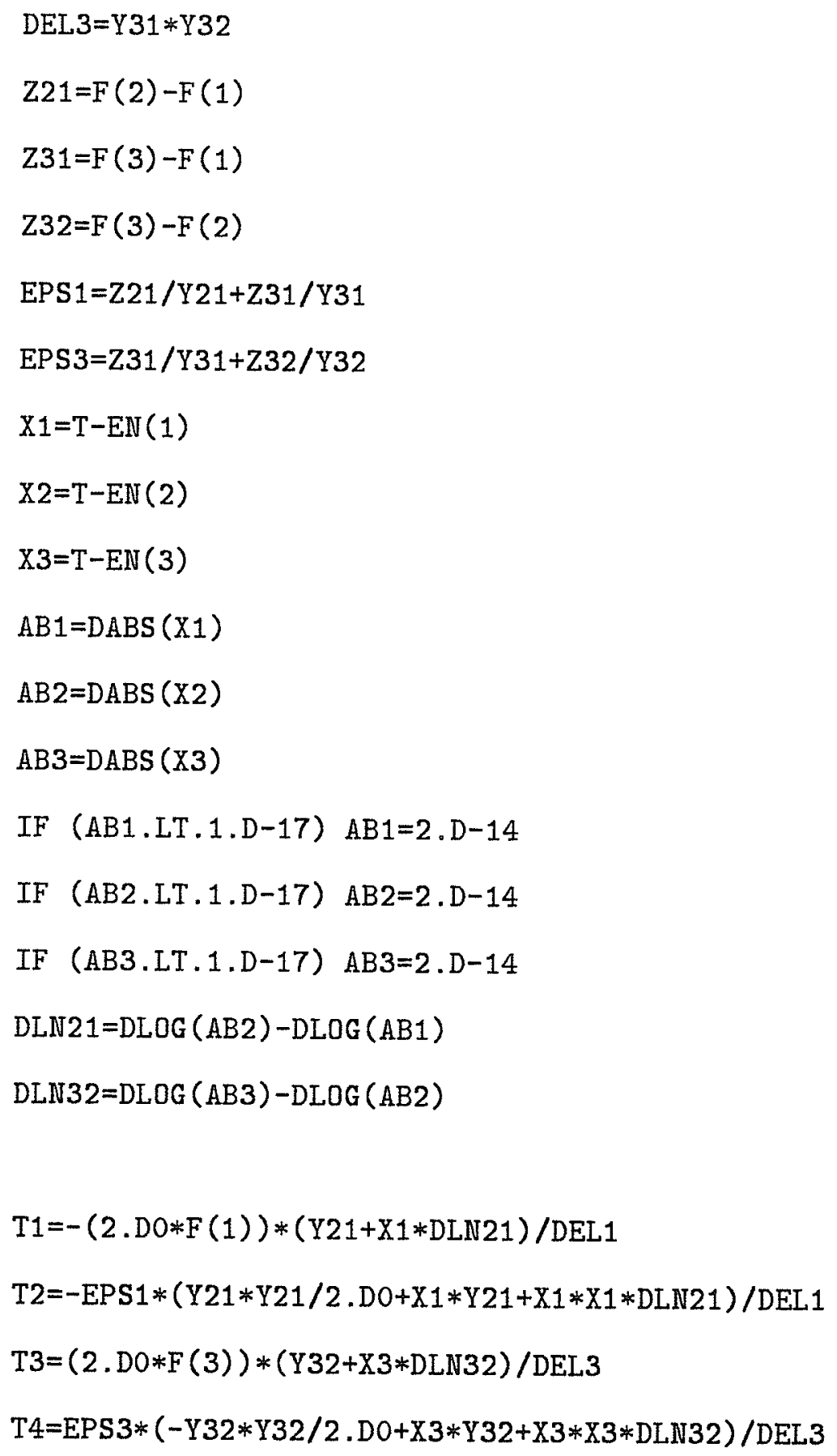


C

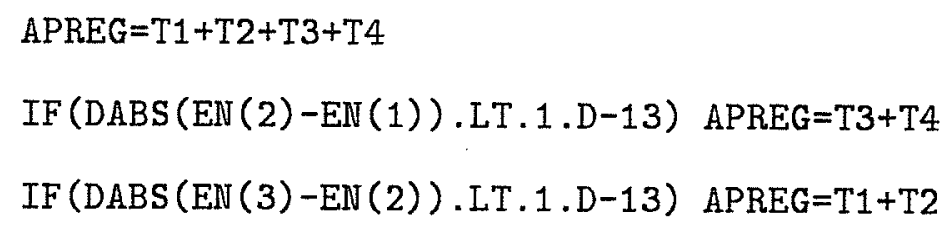

C

IF(T.IT.EN(1).OR.T.GT.EN(3)) GO TO 5

IF(T.GT.EN(2)) GO TO 10

$$
A P I M G=-(P I * X 1) *(2 . D 0 * F(1)+X 1 * E P S 1) / D E L 1
$$

GO TO 5

10 $\mathrm{APIMG}=(\mathrm{PI} * \mathrm{X} 3) *(2 . \mathrm{DO} * \mathrm{~F}(3)+\mathrm{X} 3 * \mathrm{EPS} 3) / \mathrm{DEL} 3$

C

5 RETURN

END

C

C

C

SUBROUTINE SORT

C

IMPLICIT REAL*8 (A-H, O-Z)

DIMENSION EN $(1: 3), F(1: 3)$

COMMON /ENF/EN, F

C 


$$
\begin{aligned}
& \text { D0 } 10 \mathrm{~J}=1,2,1 \\
& \text { D0 } 10 \mathrm{I}=1,2,1
\end{aligned}
$$

C

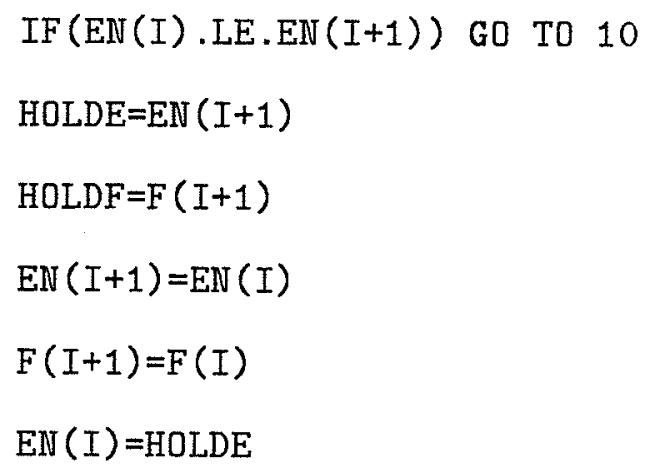

END 


\section{Bibliography}

[1] Joseph Needham. The Grand Titration. University of Toronto Press, Toronto, Canada, 1969.

[2] Shigeru Nakayama and Nathan Sivin. Chinese Science-explorations of an ancient tradition. The MIT Press, Cambridge, MS, U.S.A., 1973.

[3] Daniel. C. Mattis. Theory of Magnetism. Harper and Row, New York, first edition, 1965. International student reprint.

[4] W. Heisenberg. Z. Phys., 38:441, 1926.

[5] P.A.M. Dirac. Proc. Roy. Soc., 112A:661, 1926.

[6] G. A. Baker, H. E. Gilbert, et al. Physics Letters, 20:146, 1966.

[7] William K. Scott. The Bethe Ansatz Approach to the Two-Magnon Problem for Finite Anisotropic Ferromagnetic Chains of Arbitrary Spin. Master's thesis, University of Manitoba, Winnipeg, Canada, 1987. (see Appendix A).

[8] P. D. Loly and A. A. Bahurmuz. Journal of Physics C: Solid State Physics, 19:2241-2252, 1986. 
[9] E. Schrödinger. Proc. R. Ir. Acad., 47:39, 1941.

[10] F. Keffer. Spin Waves. In H. J. P. Wijn, editor, Handbuch der Physik, volume 18, pages 1-273, Berlin, Federal Republic of Germany, 1966. Springer-Verlag.

[11] Daniel C. Mattis. Theory of Magnetism I: Statics and Dynamics. SpringerVerlag, Berlin, Germany, 1981.

[12] H. Bethe. Z. Phys., 71:205, 1931.

[13] F. J. Dyson. Physical Review, 102:1217,1230, 1956.

[14] M. Wortis. Physical Review, 132:85, 1963.

[15] Takashi Tonegawa. . Supplement of the Progress of Theoretical Physics, 46:61$83,1970$.

[16] R. Silberglitt and A. B. Torrance. Physical Review B, 2:772, 1970.

[17] D. Pink and P. Tremblay. Canadian Journal of Phys., 50:33, 1974.

[18] S. T. Chiu-Tsao, P. M. Levy, and C. Paulson. Physical Review B, 12:1819, 1975.

[19] R. G. Boyd and J. Callaway. Physical Review A, 138:1621, 1965.

[20] P. D. Loly and B. J. Choudhury. Physical Review B, 13:4019, 1976.

[21] P. D. Loly. Canadian Journal of Physics, 65(10):1272-1279, 1987.

[22] P. D. Loly and A. A. Bahurmuz. Physical Review B, 21(5):1924, 1979. 
[23] P. D. Loly and A. A. Bahurmuz. Physical Review B, 22(3):1294, 1979.

[24] P. D. Loly and M. Hood. Journal of Physics C: Solid State Physics, 19:4729$4740,1986$.

[25] G. C. Psaltakis, G. Mischler, D. J. Lockwood, and M. G. Cottam. One- and Two-Magnon Excitations in $\mathrm{FeBr}_{2}$. Journal of Physics C: Solid State Physics, $17: 1735-1752,1984$.

[26] Veit Elser. Physical Review Letters, 62(20):2405-2408, 1989.

[27] Koh Wada, Takuma Ishikawa, and Takehiko Oguchi. . Progress of Theoretical Physics, 54(5):1589-1598, December 1975.

[28] B. W. Southern and T. S. Liu. Physical Review, 39(16):160-164, June 1989.

[29] D. N. Zubarev. Soviet Physics Uspekhi, 3(3):320, 1960.

[30] L. van Hove. Physical Review, 89:1189-1193, 1953.

[31] A. A. Bahurmuz and P. D. Loly. Physical Review B, 19(11):5803-5809, 1979.

[32] P. D. Bahurmuz, A. A.and Loly. Journal of Physics C: Solid State Physics, $20: 6277-6285,1987$.

[33] Tsuyoshi Horiguchi. Journal of Mathematical Physics, 13(9):1411, 1972.

[34] Tohru Morita and Tsuyoshi Horiguchi. . Numerische Mathematik, 20:425-430, 1973. 
[35] Roland Bulirsch. . Numerische Mathematik, 7:78-90, 1965.

[36] E. N. Economou. Green's Functions in Quantum Physics. Solid-State Sciences 7. Springer-Verlag, New York, 2 edition, 1983.

[37] P. D. Loly and J. A. Ashraff. Journal of Physics C:Solid State Physics, 20:4823$4831,1987$.

[38] Michael G. Cottam and David J. Lockwood. Light Scattering in Magnetic Solids. John Wiley \& Sons, Toronto, 1986.

[39] I. W. Johnstone, D. J. Lockwood, and G. Mischler. Journal of Physics C: Solid State Physics, 11:2147-2164, 1978.

[40] D. J. Lockwood et al. Journal of Physics C: Solid State Physics, 15:2973-2992, 1982. 